19-8 rils

(x)

kit wort gorst

is. 3 :

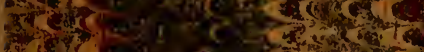

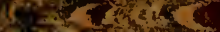

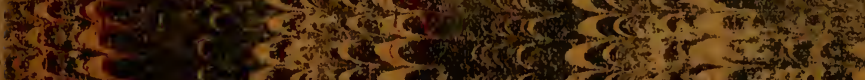

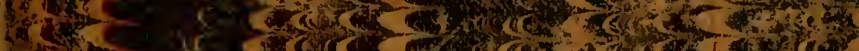

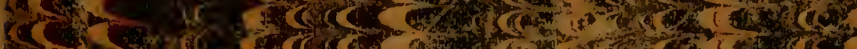

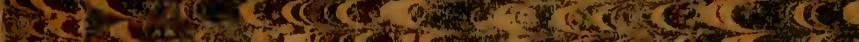

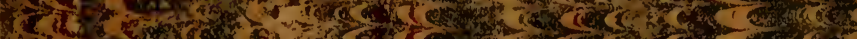

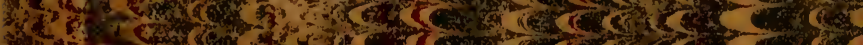
2.

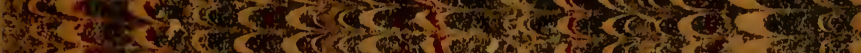

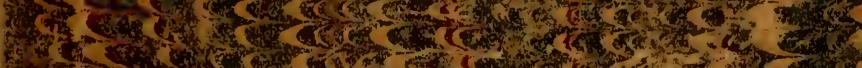
8.

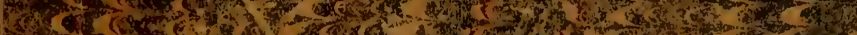

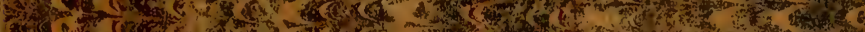
-

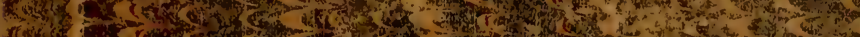
Xos

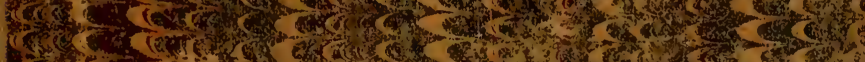

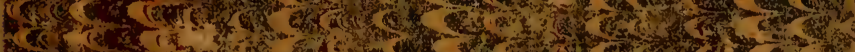
If

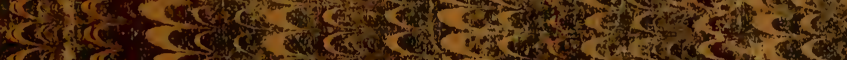

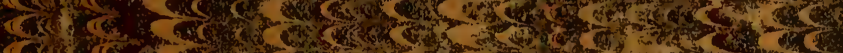

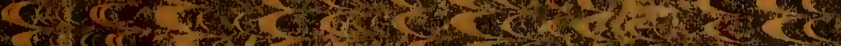

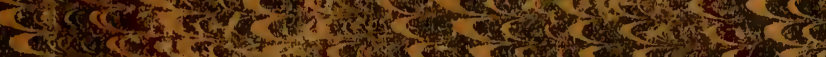

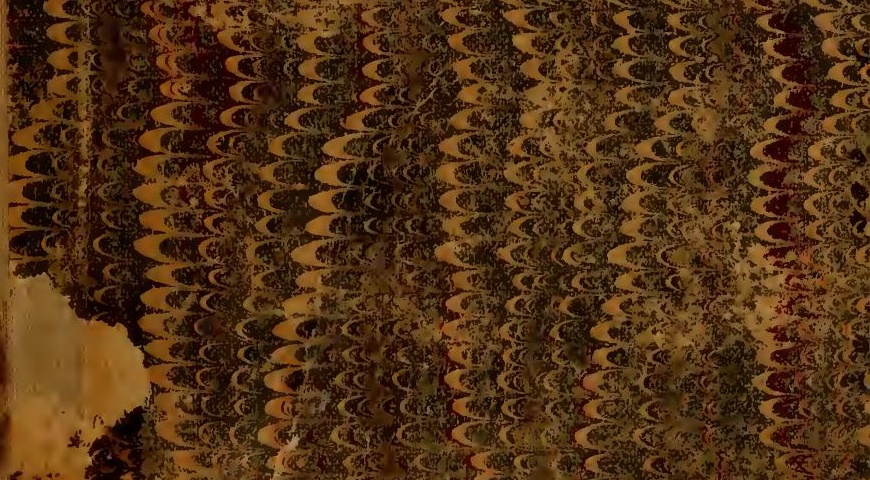

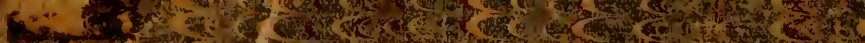

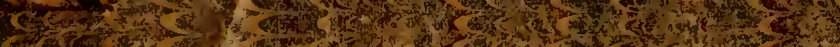



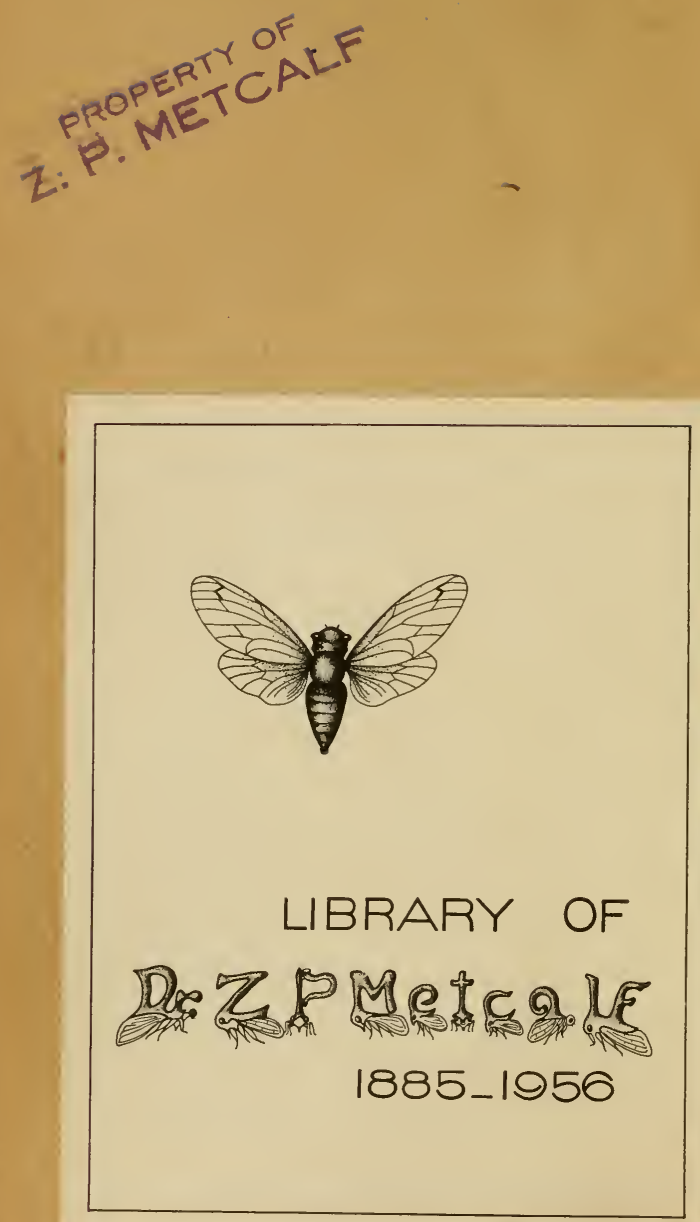




\title{
LES
}

\section{INSECTES PHOSPHORESCENTS}

\author{
NOTES COMPLÉNENTAIRES \\ E'T

\section{BIBLIOGRAPHIE GÉNÉRALE}

(ANATONIE, PHYSIOLOGIE ET BIOLOGIE)

=urros 



\title{
LES
}

\section{INSECTES PHOSPHORESCENTS}

\section{NOTES COMPLÉMENTAIRES}

\author{
E T \\ BIBLIOGRAPHIE GÉNÉRALE \\ (ANATOMIE, PHYSIOLOGIE ET BIOLOGIE)
}

PAR

Henri GadeaU de KerVille

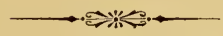

\author{
ROUEN \\ IMPRIMERIE JULIEN LECERF \\ 1887
}




\section{PRINCIPAUX TRAVAUX DU MÊME AUTEUR.}

Les Insectes phosphorescents, avec '́ pl. chromolithographièes. Rouen, Léon Deshays, 1881.

Comptes rendus des $19^{\mathrm{e}}, 20^{\circ}, 21^{\circ}, 22^{\mathrm{e}}, 23^{\mathrm{e}}$ et $2 \mathbf{4}^{\mathrm{e}}$ réunions des Délégués des Sociètés savantes à la Sorbonne (Sciences naturelles), 1881, 1882, 1883, 1884, 1885 et 1886, in Bull. de la soc. des Amis des Scienc. natur. de Rouen, $1^{\text {er }}$ sem. des années 1881, 1882, 1883, 188', $188 \%$ et 1886. (L'avant-dernicr avec $3 \mathrm{pl}$. eu hèliogravure et $1 \mathrm{pl}$. en couleur).

Recherches physiologiques et hi:tologiques sur l'organe de l'odorat des Insectes, par Gustave Hauser, d'Lrlangren (Bavière), traduit de lallemand, avec 1 pl. lithographiée, in Bull. de la Soc. des Amis des Scienc. natur. de Rouen, $1^{\text {er }}$ sem, 1881 .

Liste générale des Mammifères sujets à l'allinisme, par Elvezio Cantoni, traduction de l'italien et additions, in Bull. de la Soc. des Amis des Scienc. natur. de Rouen, 1er sem. 1882.

Les ceufs des Coléoptères, par Mathias Rupertsberger, traduit de l'allemand, in Revue d'Éntomologie, ann. 1852.

De laction du mouron rouge sur les Oiseaux, in Compt. rend. hebdom. des séanc. de la Soc. de Biologie (séance du 8 jullet 1882).

De l'action du persil sur les Psittacides, in Clompt. rend. hebdom. des séanc. de la Soc. de Biologie (séance du 20 janvier 1883).

De l'action du persil sur les Psittacidés (nouvelles expériences et notes complémentaires). Rouen, Léon Deshays, 1883.

De la structure des plumes et de ses rapports avec leur coloration, par le Dr Hans Gadow, de Cambridge (Angleterre), traduit de l'anglais et annoté, avec 1 pl. lithographiće, in Ball. de la Soc. des Amis des Scienc. natur. de Rouen, fer sem. 1883.

Sur la manière de décrire et de représenter en couleur les animaux a reflets métalliques, avee fig. dans le texte, in Bull. de l'Associat. franç. pour l'Avincement des Sciences, Congrès de Rouen, ann. 1883.

Mélanges entomologiques, 3 mémoires, Ier sem. 1883, 20 sem. 1883, et $1^{\text {cr }}$ et 2e sem. 1884, in Bull. de la Soc. des Amis des Scienc. natur. de Rouen, $1^{\text {er }}$ sem. 1883, 2e sem. 1883, et 2e sem. 188́t.

Les Myriopodes de la Normandic (1 re liste), suivie de Diagnoses d'Es- 
péces et de Varietés nouvelles, par le Dr Robert Latzal, de Vienne (Autriche), avec 1 pl. lithographiée, in Bull. de la soc. des Amis des Scienc. natur. de Rouen, 2 sem. 1883.

Les Myriopodes de la Normandie (2e liste), suivie de Diagnoses d'Espéces et de Variétés nouvelles (rle France, Algérie et Tunisie), par le $D^{r}$ Kobert Latzel, de Vienne (Autriche), in Bull. de la Soe. des Amis des Scicne. natur. de Rouen, 20 som. 1883.

Note sur une Espéce nouvelle de Champignon entomogène (Stilbum Kervillei, Quélet), avec 1 pl. en couleur, in Bull. de la Soc. des Amis des Scienc. natur. de Romen, 2e sein. 1883.

Note sur un Orque Epaulard pêché aux environs du Tréport, in Bull. de la Soc. des Amis des Seienc. natur. de Rouen, Ier sem. $188 \dot{\text { t. }}$

De la reproduction de la Perruche Soleil (Conurus solstitialis, Less.) en France, in Bull. mensuel de lis Soc. nation. d'Acclimatition de lirance, $\mathrm{n}^{\circ} \mathrm{T}$ (juillet) de 1884.

Note sur un Canard monstrueux appartenant au genre Pygomèle, avec 1 pl. lithographice, in Journ. de l'Anatomie et de la Physiologie, $n^{\circ} 5$ (septembre-octobre) de 1884.

Description de quatre Monstres doubles (2 Chats et 2 Poussins) aprartenant aux genres Synote, Iniodyme, Opodyme et Ischiomèle, avec 1 pl. lithographièe, in Journ. de l'Anatomie et de la Physiologie, $n^{\circ} 4$ (juillet-aoùt) de 18:3.

Note sur les Crustacés Schizopodes de l'Estuaire de la Scine, suivie de la description d'une Espèce nouvelle de Mysis (Mysis Fervillei, G. O. Sars), par G. O. Sars, de Cihristiania (Norwége), avec 1 pl. gravée, in Bull. de la Soc. des Amis des Seienc. natur. de Ronen, ler sem. $188 \%$.

Note sur un hybride bigènère de Pigeon domestique et de Tourterelle à collier, suivie de la Récapitulation des hybrides uni-et tigénères obseriès jusqualors dans l'Ordre des Pigeons, in Bull. de la Soc. des $\Lambda$ mis des Scienc. natur. de Rouen, $2^{e}$ sem. 188.

Aperçu de la Faune actuelle de la Seine et de son embouchure, depuis Rouen juzqu'au Havre, in 2 vol. de L'Estuaire de la Seine, par G. Lennier. Le Harre, impr. du journal Le Havre, 188 3.

La Faune de l'Estuaire de la Seine, in Annuaire normand, ann. 1886.

Causeries sur le Transformisme. Paris, C. Reinwald, 1887.

Etc., etc. 



\section{AVANT-PROPOS}

Il y a six ans, j’ai publié sur Les Insectes phosphorescents ${ }^{1}$ un modeste ouvrage de vulgarisation, dans lequel j'indiquais les particularités les plus intéressantes de la structure et de la biologie de ces animaux, et où je donnais, dans quatre planches chromolithographiées, la reproduction des types les plus caractélistiques de cos Insectes si curieux. Cet ouvrage était l'un de mes débuts dans la science; c'est dire qu'il est fort imparfait, et qu'à chaque page on y rencontre l’inexpérience forcée du néophyte.

Il est rare, d'ailleurs, qu'un savant soit content de ses premiers essais - si toutefois un savant peut jamais être complètement satisfait de l'un quelconque de ses ouvrages. Lorsqu'un auteur, en relisant ses premier's travaux, s'apercoit qu'il a indiqué des faits inexacts, qu'il a commis des fautes de style plus ou moins grossières, résultat fatal de l'inexpérience, il n’a généralement qu’un désir', malheureusement irréalisable : celui d'anéantir ses premières auvres, pour les recommencer à nouveau. J'ai maintes fois éprouvé ce désir, mais ne pouvant le satisfaire, j’ai dì prendre le parti, en cette circonstance, de donner un supplénent

1. Voir lia note de la page 11. 
à mes Insectes phosphorescents, sous forme de Noles complémentaires. Ces Notes sont aussi courtes que possible; elles renferment seulement l'indication des inexactitudes que j’ai laissées passer dans le mémoire en question, jointe à plusieurs faits nouveaux. Je n'ai pas voulu leur donner une extension plus grande, tenant à conserver à mon travail son cachet d'ouvrage élémentaire.

Depuis quelques années, j’ai réuni avec le plus grand soin les titres de tous les mémoires, travaux et notes publiés sur l'anatomie, la physiologie et la liologie des Insectes phosphorescents, à partir d'Aristute jusqu'à aujourd'hui. Tous ces titres forment la Bibliographie générale, qui fait le fond de ce travail. J'ai l'espoir que cette bibliographie sera utile à ceux qui se livreront à l'étude de la phosphorescence des Insectes, question physiologique des plus intéressantes, qui, en dépit des nombreuses recherches qu'elle a suscitées, réserve encore beaucoup de faits nouveaux aux savants de l'avenir. 
NOTES COMPLÉMENTAIRES 



\section{NOTES COMPLEMENTAIRES}

\section{GÉNÉRALITÉs.}

1

P. 7, 1. 14. "Plus de cent mille sont décrits aujourd'hui ». - Depuis l'époque où j'écrivais ces lignes, plusieurs milliers d'espèces de Coléoptères ont été décrites; et, chaque jour, les naturalistes et les explorateurs en décourrent de nouvelles. Le nombre de cent mille est donc insuffisant pour indiquer la quantité d'espèces de Coléoptères actuellement connues. Toutefois, il n'est pas douteux, selon moi, que les entomologistes futur's relègueront une grande quantité de formes, décrites comme de véritables espèces, au rang de simples races ou variétés. En outre, un chiffre très-élevé de noms nouveaux sont destinés à grossir le nombre considérable des synonymes que posside la science entomologique.

P. 8, 1. 7. - Cette phrase est incorrocte. Au lieu de : les Coléoptères " passent, avant d'atteindre leur entier développement, par les trois états de larve, de nymphe et d'Insecte parfait », lire : les Coléoptères passent par les trois états successifs d'œuf, de larve et de nymphe, avant d'arriver ì l'état d'Insecte parfait.

1. Henri Gadeau de Kerville. - Les Insectes pho phorescents, avec \& $\mathrm{pl}$. chromolithographiées. Rouen, Lóon Deshays, 1881. 


\section{COLÉOPTÉRES.}

\section{Elatérides.}

P. 12, 1. 11. - Des études approfondies sur les Elatérides de la sous-tribu des Pyrophorites ont conduit it mettre en synonymie une certaine quantité de noms d'espèces, et à faire connaitre plusieurs espèces nourelles. Il résulte de ces études que l'on peut éraluer à environ soixante-treize le nombre des espèces actuellement connues de la sons-tribu des Pyrophorites, dont soixante-dix appartiennent all genre Pyrophorus et trois au genre Pliotophorus.

P. 12, 1. 12. - Aux pays indiqués. il faut ajouter les EtatsUnis, au vit le P'yrophorus physoderus, Germ., qui, de toutes les espèces du genre, est celle dont l'habitat est le plus septentrional.

Les espèces du genre Pyrophorus sont propres aux deux Amériques, particulièrement ì l'Amérique méridionale et aux Antilles; celles du genre Photophorus virent dans quelques îles océaniennes.

P. 12, 1. 16. - Les øufs, les larves, et sans doute les nymphes, sont phosphorescents.

Quelques naturalistes ont fait mention de larves lumineuses d'Elatéricles appartenant ì des groupes autres que celui des Pyrophorites. A cet égard. je renroie le lecteur au mémoire de Raphaël Dubois ${ }^{1}$ sur Les Elutérides lumineux, où cette question est soigneusement traitée (p. 11 et suiv.), et à la Bibliographie générale, dans laquelle il trouvera, soit dans le titre des traraux, soit dans les renseignements mis entre crochets, l’indication des larves

1. Voir la Bibliographie genérale. 
lumineuses considérées comme appartenant à des Elatérides.

P. 12, 1. 20. - L'expression de " angles inférieurs du prothorax " prête ì la confusion et doit être remplacée par celle de " angles supéro-postérieurs du prothorax ".

P. 12, I. 21. - Dans son remarquable travail sur Les Elatérides lumineux, Raphaël Dubois ${ }^{1}$ dit, en parlant du troisième appareil phosphorique des Pyrophores (p. 67): " En réalité, l'appareil lumineux ventral n'a aucun rapport avec le thorax, il est une dépendance absolue du premier segment abdominal : il occupe la région intermédiaire du sternite du premier zonite de l'abdomen. Le tégument de la région qu'il occupe est moins chitinisé que celui de la région avoisinante, de façon à demeurer transparent ».

P. 14, 1. 21. - La propriété lumineuse du prolongement céphalique du Fulgore Porte-lanterne (Fulgora laternaria, L.), était connue en Europe avant la publication du livre de Sibylle Mérian, car, en 1681, Grew ${ }^{1}$ en parlait déjà dans son Juseum regalis societatis. Il est donc possible que ce soient les Fulgores que Fontenelle ait confondus avec des Oiseaux.

P. 16, 1. 27. - Au lieu de Bondazoy, lire Fougeroux de Bondaroy.

Pour toutes les questions relatives à l'anatomie, la physiologie, la biologie, etc. des Pyrophores, ainsi qu'à la structure et au mode de fonctionnement de leurs organes lumineux, je renvoie le lecteur à l'admirable travail de Raphaėl Dubois ${ }^{1}$ sur Les Elatéricles lumineux, où ces questions sont traitées d'une façon magistrale.

1. Voir la Bibliographie générale. 


\section{Malacodermes.}

P. 29, 1. 10. - Au lieu de "vient du grec ky.nupis, qui signifie lampe ", - étymologie erronée, car lampe se dit en

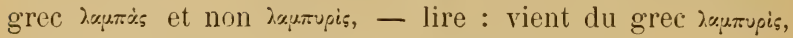

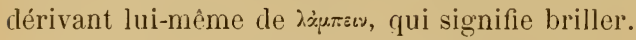

P. 30, 1. 9. - Angles postérieurs au lieu de : angles inférieurs.

P. 30, 1. 16. - Le Lampyre noctiluque habite presque toute l'Europe et l'Asie ainsi que le Nord de l'Afrique, et non pas tout l'univers.

P. 30, l. 30. - On sait aujourd hui que l'œuf, la larve, la nymphe et les adultes (màle et femelle) du Lampyre noctiluque sont phosphorescents; le mâle étant lumineux par luimême, mais à un degré beaucoup plus faible que la femelle.

\section{Carabides, Staphylinides, Pausides, Buprestides, Ténébrionides et Cérambycides.}

Quelques auteurs ont signalé, en dehors des deux familles des Elatérides et (les Malacodermes, qui renferment les Coléoptères lumineux, différents Coléoptères appartenant à d'autres familles, chez lesquels on aurait observé, parait-il, des phénomènes de phosphorescence.

Dans les lignes suivantes, j’indique les quelques observations que j’ai pu recueillir à cet égard, sans vouloir nullement prétendre qu'il n'en ait pas été fait d'autres sur ce sujet. A mon avis, la plupart de ces observations, sinon toutes, sont erronées. Il se peut que l'on ait observé des phénomènes de phosphorescence chez les animaux en questiun, mais cette 
apparence lunineuse levait ètre due à un corps phosphorescent quelconque, soit à un fragment d'un animal lumineux, soit à une parcelle de viande en décomposition, de bois pourri, de certains Champignons, corps qui émettent parfois, comme on le sait, une lumière phosphorescente, soit à un autre objet phosphorescent. Je crois que pas un des Coléoptères suivants n'est lumineux par lui-ınème, et que les différents faits relatifs ¿̀ leur prétendue phosphorescence doivent être laissés complètement de côté, tant qu'ils n'auront pas été confirmés par de nouvelles et sérieuses observations :

Voici les faits en question :

Vestwood ${ }^{1}$ signale des phénomènes de phosphorescence chez un Vebria (Helobia) brevicollis, Fabr., Coléoptère de la famille des Carabides, en faisant observer que cette apparence lumineuse était due, selon lui, à un corps phosphorescent quelconque qui adhérait à cet Insecte.

Reiche $^{1}$, Parzudaki ${ }^{1}$ et Rouzet ${ }^{1}$ donnent des détails sur la crépitation des Brachinus, Coléoptères de la famille des Carabides, et signalent la lueur phosphorescente qui accompagne cette crépitation.

Georg’e, H. jun. ${ }^{1}$ dit avoir trouvé un Staphylinus (Goërius) olens, Mïiller, Coléoptère de la famille des Staphylinides, qui était phosphorescent.

Peragallo ${ }^{1}$ relate une observation qu'il a faite à Menton sur un Insecte en tout semblable ḋ un Staphylinus olens, Müller de forte taille, qui laissait derrière lui une trace lumineuse. Il admet (p. 663) : « ou qu'il existe à Menton un Staphylin phosphurescent vivant de Lucioles », ce que je ne puis

1. Voir la Bıbliographie générale. 
croire, ou que les individus rencontrés par lui et son compagnon de chasse " se trouvaient enduits de la pate phosphorescente et très-persistante qui emplit les deux derniers anneaux de l'abdomen des Lucioles qu'ils venaient de manger "; cette dernière explication me parait seule admissible.

Afzelius ${ }^{1}$ prétend que les massues creuses des antennes du Pausus sphaerocerus, Afz., Coléoptère de la famille des Pausides, émettent une faible lumière phosphorescente. Par contre, dans sa Monographie de ce genre d'Insectes, Westwood $^{2}$ attribue cette apparence lumineuse, non à un phénomène de phosphorescence, mais plutôt au jeu de la lumière sur la surface extrèmement polie de la massue globuleuse des antennes de cet Insecte. J'ajouterai que Péringuey ${ }^{3}$, dans ses Notes on three Paussi, déclare que la phosphorescence des antennes n`a été olservée chez aucune des trois espèces étudiées (Pausus linealus, Thunbg.; P. Linnei, Westw.; et $P$. Burmeisteri, Westw.).

Latreille $^{1}$ fait mention, d'après l'un de ses amis, de la phosphorescence de la grande tache ocellée qui se trouve sur chacune des élytres du C'hrysochroa (Buprestis) ocellata, Fabr., Coléoptère de la famille des Buprestides. Cette assertion est sans nul doute erronée.

Lamarck $^{-1}$ dit que les deux taches orales et rouges, recouvertes d'une membrane pubescente, qui se trouvent sur le

1. Voir la Bibliographie générale.

2. John-Obadiah Westwond. - On the Paussidae, a family of Coleopterous Insects, in Transact. of the Linn. Soc. of London, ann. 1833, p. 607, av. $1 \mathrm{pl}$.

3. Louis Péringuey.-Notes on three Paussi, in 'Transact. of the entomol. Soc. of London, ann. 1883, p. 133. 
second segment de l'abdomen du Chiroscelis bifenestrata, Lam., Coléoptère de la famille des Ténébrionides, indıquent un organe particulier, peut-être un organe phosphorescent.

Kirby ${ }^{1}$ et Spence ${ }^{1}$, dans leur Introduction to Entomology, disent (p. 508) que Laporte a informé Chevrolat qu'un nombre considérable d'Hélopides brésiliens (Coléoptères de la famille des Ténébrionides), alliés aux stenochia, ont des segments abdominaux de couleur et d'apparence semblables aux segments abdominaux phosphorescents des Lampyrides, ce qui indiquerait une même propriété lumineuse.

Kirby ${ }^{1}$ et Spence $^{1}$ rapportent aussi (loc. cit.) que le Dadoychus flavocinctus, Chev., Coléoptère de la famille des Cérambycides, allié aux Saperda, possède un abdomen dont les troisième et quatrième segments ont la même couleur jaune et le même aspect que les segments lumineux des Lampyriıles, d'où Chevrolat conclut que cet Insecte est phosphorescent.

Les assertions de Lamarck, de Laporte et de Chevrolat n'ont jamais été sanctionnées par l'observation et l'expérience.

Enfin, Luce ${ }^{1}$ a décrit un scarabaeus phosphoricus qui, probablement, n'est autre que la Luciola italica, L. ou la L. lusitanica, Charp.

\section{HÉMIPTĖRES.}

P. 41, 1. 21. - La question de la phosphorescence des Fulgor $a$ et genres voisins est loin encore d'ètre résolue. En effet, tandis que plusieur's naturalistes affirment que le prolongement céphalique de ces Insectes émet une lueur phos-

1. Voir la Bibliographie génèrale. 
phorescente, d'autres prétendent n'avoir jamais pu constater ce fait, bien qu'ils aient observé attentivement ces animaux, ì l'état libre et en captivité. Peut-être l'un des deux sexes est-il seul lumineux? Peut-être les deux sexes le sont-ils, mais à une certaine époque de l'année. Peut-être ne sont-ils lumineux qu'au moment de l'accouplement? Peutêtre même n'y a-t-il que certains individus qui possèdent le pouvoir d'émettre une lueur phosphorescente?

Quoi qu'il en soit, il est impossible de se prononcer actuellement pour ou contre la phosphorescence des Fulgorides, admise et niée tour à tour par des personnes dignes de foir. Il faut attendre les résultats d'observations sérieuses, plusieurs fois répétées, pour résoudre, d'une manière définitive, cette intéressante question d'entomologie physiologique.

\section{ORTHOPTĖRES, DIPTĖRES, LÉPIDOPTĖRES ET HYMÉNOPTẺRES.}

Allmann ${ }^{1}$ parle de la phosphorescence de l'Anurophorus fimetarius, Nicolet, Orthoptère du sous-ordre des Thysanoures et de la famille des Podurides.

Kirby ${ }^{1}$ et Spence ${ }^{1}$, dans leur Introduction to Entomo$\operatorname{logy}$ (p. 510), disent qu'on aurait trouvé en Angleterre un individu lumineux de la Courtilière (Gryllotalpa vulgaris, Latr.), Orthoptère de la famille des Gryllides. Cette assertion doit être complètement erronée.

Eaton ${ }^{1}$, Lewis ${ }^{1}$ et Waterhouse ${ }^{1}$ signalent la phosphorescence du Teloganodes tristis, Hag., ơ, Orthoptère pseudoNeuroptère de la famille des Ephémérides.

1. Voir la Bibliographie générale. 
Eaton ${ }^{1}$ et Hagen ${ }^{1}$ parlent de la phosphorescence d'une autre espèce d'Ephéméride, le Caenis dimidiala, Steph., o*.

Wahlberg.' signale la phosphorescence des larves et des nymphes d'un Diptère rle la famille des Mycétophilides, le Ceroplatus sesioüdes, Walılbg.

Hudson ${ }^{1}$ et Osten-Sacken ${ }^{1}$ mentionnent la phosphorescence de larves d'un Diptère de la Nouvelle-Zélande, qui appartient très-probablement ì la famille des Mycétophilides.

Osten-Sacken ${ }^{1}$ parle de larves lumineuses de Chironomus. (Diptères de la famille des Chironomides).

Swinton ${ }^{1}$, dans son Insect Variety, mentionne (p. 101) le Chironomus tendens, Fabr., Diptère de la famille des Chironomides, dont le thorax et l'abdomen seraient, parait-il, lumineux. Le même auteur signale aussi (loc. cit.) un Diptère de la famille des Tipulides, une Tipula? chez laquelle on aurait observé des plıénomènes de phosphorescence.

Main 'signale un Insecte lumineux, abattu par un fermier et décrit par lui comme se rapportant exactement à une Tipula oleracea, L. ${ }^{2}$, Diptère de la famille des Tipulides.

Pallas ${ }^{1}$ fait mention d'un Culex lumineux. (Diptère de la famille des Culicides).

Robineau-Desvoidy ${ }^{1}$, Girard ${ }^{1}$, etc., font mention d'un Diptère de la fanille des Muscides, le Thyreophora cynophila, Panz., dont la tête émet la nuit une lueur phosphorescente.

Boisduval ${ }^{1}$ parle de la phosphorescence accidentelle de la

1. Voir la Bibliographie générale.

2. Voir, au sujet de cette prétendue Tipule lımineuse, Kirby et Spenco. (Op. cit., p. 511). 
chenille de la Mamestra oleracea, L., Lépidoptère de la famille des Hadénides.

Gimmerthal ${ }^{1}$ signale des faits de phosphorescence chez la chenille de l'Agrotis (Noctua) occulta, L., Lépidoptère de la famille des Agrotides.

Tiedemann ${ }^{1}$ dit que, selon Brown, le Pyralis minor, Microlépidoptère de la famille des Pyralides, a l'abdomen faiblement lumineux. Ce fait me semble complètement inadmissible. (Voir suppl., p. 171.)

Enfin, Villiers ${ }^{1}$ fait mention de petites Fourmis jaunes qui auraient présenté des phénomènes de phosphorescence; mais cette prétendue observation d'Hyménoptères Formicides lumineux doit être complètement erronée.

\section{PHYSIOLOGIE.}

P. 48, 1. 3. - Au lieu de Humprey Davy, lire Humphry Davy.

P. 18, 1. 20. - Au lieu de violettes, lire violets.

P. 52, 1. 29. - Depuis la publication des recherches expérimentales de Jousset de Bellesme sur la phosphorescence du Lampyre, différents travaux importants ont paru sur la phosphorescence des Pyrophores, des Lampyres et des Lucioles; mais je n'en donnerai pas l'analyse, afin de ne point dépasser le but très-modeste que je m étais proposé, en rédigeant mon premier travail sur les Insectes phosphorescents.

Toutefois, je crois utile de reproduire ici une partie des conclusions déduites par Raphaël Dubois ${ }^{2}$ des multiples et

1. Voir la Bibliographie générale.

2. Raphaël Dubois. - Les Elatérides lumineux, p. 270. (Voir la Bibliographie gẻnérale). 
remarquahles recherches qu'il a faites sur le Pyrophorus noctilucus, L. J'ajouterai que ces conclusions ne doivent ètre appliquées quaux Pyrophores, car, en fait de lumière biologique, toute généralisation est prématurée et imprudente.

Voici les plus importants des résultats obtenus par ce physiologiste distingué, résultats qui peuvent être considérés comme le dernier mot de la science actuelle sur la question de la phosphorescence des Elatérides lumineux :

"L'ètude anatomique et histologique des organes lumineux montre qu'ils sont composés d'un tissu adipeux spécial et d'organes accessoires. L'histochimie indique l'abondance, dans ce tissu, d'une substance qui présente les caractères de la guanine.

"Au sein de ce tissu adipeux photogène s'effectuent des phénomėnes d'histolyse intense, provoqués ou activès par la pénétration du sang dans l'organe lumineux.

"Ce processus histolytique est accompagné de la formation, au sein même de la cellule photogène, d'une innombrable quantité de petits conglomérats cristallins doués de propriétés optiques particulières et spécialement d'une biréfringence très-accentuée.

" L'intervention du sang n'est pas indispensable à l'accomplissement du phénomène lumineux, car l'œuf est luisant, même avant la segmentation. La cellule adipeuse photogène isolée jouit de la mème propriété, ce qui établit un nouveau rapprochement entre la substance du corps adipeux et celle du vitellus.

"Les muscles des appareils lumineux règlent l'apport du sang dans les organes photogènes et agissent ainsi indirectement sur la production de la lumière.

"C'est par l’intermédiaire des muscles que les nerfs 
interviennent dans l'accomplissement de la fonction photogénique. Le réflexe photo-sensitif a son siège dans les ganglions cérébroïdes. L'excitation descendante ou centrifuge des ganglions d'où émanent les nerfs des appareils lumineux, provoque, de même que leur excitation directe, l'apparition de la lumière. Il n'en est pas de même si l'excitation est centripète ou ascendante. Le cerveau commande aux appareils lumineux par le moyen des nerfs qui animent les muscles striés spéciaux.

"La respiration n'exerce qu'une influence indirecte sur la fonction photogénique, en maintenant l'intégrité des conditions de vitalité des tissus et d'activité du sang.

" La nature de l'alimentation est sans influence sur la production de la lumière animale.

“ La cellule (œuf non segmenté, cellule adipeuse), sous l'influence de la nutrition, prépare les principes photogènes, mais la lumière n'est pas le résultat direct de l'activité propre de l'élément anatomique organisé et vivant.

"Lorsque la structure de l'élément anatomique et sa vitalité ont été détruites, le phénomène lumineux peut se produire encore par une action physico-chimique de même ordre que celle qui transforme le glycogène en sucre, dans l'élément hépatique, par exemple.

"L'analogie est frappante, dit Raphaël Dubois (p. 267), entre le phénomène physico-chimique qui provoque l'apparition de la lumière dans la cellule lumineuse et ce qui se passe au sein de l'élément hépatique, dans la fonction glycogénique.

" Ces phénomènes sont absolument de même ordre, bien que différents par les substances mises en présence et le résultat final de la réaction. 
"Nous sommes bien loin déjì des explications basées sur la contraction musculaire, l'influx nerveux, l'électricité, la phosphorescence proprement dite, la combustion photogène, etc., etc.

"Il s’agit bien ici d'une double réaction d'ordre chimique s'opérant, au sein mème de la cellule, entre les produits de sa destruction physiologique.

" Le rôle du sang lui-même, auquel Heinemann attribue hypothétiquement la plus grande importance, n'est que secondaire. Il est facile de prouver que ce liquide n'intervient pas directement dans la réaction d'où nait la lumière, car on ne peut ranimer l'éclat de la substance qui a cessé de briller, en y ajoutant du sang pris dans l'organe même.

" Il est à noter toutefois que cette réaction chimique nécessite l'intervention d'un ferment soluble et coagulable, c'est-à-dire d'une de ces substances singulières qui ont bravé jusqu'à présent, non-seulement la synthèse, mais même l'analyse, dont l'origine est dans la substance organisée et dont l'intervention semble nécessaire à l'activité de tous les êtres vivants, sans en excepter les ferments figurés euxmêmes.

" Est-ce à dire que le déterminisme du phénomène qui engendre la lumière ne puisse être poussé plus loin encore, alors même que les éléments de la réaction chimique auraient pu être isolés et définis?

"En aucune façon, car un autre problème se pose inmédiatement, et l'on est en droit de se demander si la lumière est produi:e par l'énergie de la réaction elle-mème ou bien si cette réaction, qui est accompagnée, au sein des tissus, de l'apparition de myriades de corpuscules cristallins, n'en- 
gendre pas la lumière secondairement, par le fait mểme de la cristallisation qu'elle semble provoquer.

"Quoi qu'il en soit, nous sommes parvenus à réduire la fonction photogénique, chez les Elatérides lumineux, à un phénomène physico-chimique et à déterminer sa nature ainsi que la catégorie à laquelle il appartient.

" Le problème entre maintenant dans une phase nouvelle et sort du domaine de la physiologie proprement dite.

" L'œuvre du physiologiste est terminée, disait Claude Bernard, quand un phénomène biologique est réduit à l'état de phénomène physico-chimique ». 


\section{BIBLIOGRAPHIE GÉNÉRALE}

DES

\section{INSEGTES PHOSPHORESCENTS}

(ANATOMIE, PHYSIOLOGIE E'T BIOLOGIE) 



\section{BIBLIOGRAPHIE GÉNÉRALE}

\section{DES \\ INSEGTES PHOSPHORESGENTS}

(ANATOMIE, PHYSIOLOGIE ET BIOLOGIE)

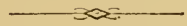

\section{INTRODUCTION}

Aujourd'hui, plus que jamais, les bibliographies générales et spéciales, relatives aux sciences biologiques, sont de la plus incontestable utilité. De tous côtés, en effet, des Sociétés d'histoire naturelle s'organisent et reçoivent, i peine créées, de nombreux adhérents; les recherches se multiplient; les travaux se succèdent sans interruption; el la science se trouve enrichie, pour ainsi dire chaque jour, d'une découverte intéressante. Mais ces travaux sont disséminés dans un grand nombre d'ouvrages, de mémoires, de bulletins, d'annales, de revues, de journaux, et il serait complètement impossible à un naturaliste de se rendre compte, même très-imparfaitement, du genre et de la valeur des travaux insérés dans ces publications, si des savants dévoués, comme les Bertkau, les Carus, les Erichson, les Giebel, les Hagen, les Paul Mayer, les Taschenberg, etc., pour n'en citer que plusieurs des principaux, n'avaient pas distrait de leurs attachantes éturles le temps énorme que nécessitent les recherches bibliographiques. Gràce à eux, gràce à leurs gigantesques compilations, grâce aux recueils 
que plusieurs d'entre eux publient chaque année, tous les naturalistes peuvent connaitre, rapidement et sans frais, les différents mémoires publiés sur le sujet qui les intéresse, et se tenir constamment au courant de leur science favorite.

Il n'est pas nécessaire d'insister plus longuement sur l'extrême utilité de ces sortes de travaux, car tous ceux qui s'occupent de questions scientifiques peuvent sans cesse la reconnaître. Aussi, n’ai-je pas cru inutile de publier la petite bibliographie ci-jointe qui, je l'espère, rendra quelques services à ceux qui voudront étudier la phosphorescence des Insectes. J'en avais réuni les premiers éléments lorsque je fis paraitre, en 1881, mon étude de vulgarisation sur les Insectes phosphorescents; depuis cette époque, je l'ai beaucoup augmentée et me suis efforcé de la rédiger aussi clairement que possible.

Dans cette bibliographie générale des Insectes phosphorescents, je n’ai indiqué, à dessein, que les mémoires, travaux, notes, etc., relatifs à l'anatomie, à la physiologie et à la biologie de ces Insectes, laissant entièrement de côté tous les travaux systématiques, qui n'eussent fait qu'augmenter inutilement l'importance de cette compilation. rendre les recherches plus difficiles, et changer complètement le but que je me suis proposé d'atteindre. D'ailleurs, les entomologistes qui voudront étudier les Insectes phosphorescents au point de vue purement descriptif, trouveront, dans les ouvrages que j'énumère ci-dessous ${ }^{1}$, les divers renseignements bibliographiques dont ils auront besoin.

1. Julius-Victor Carus et Wilhelm Engelmann. - Bibliotheca zoologica. Verzeichniss der Schriften über Zoologie welche in den periodischen Werken enthalten und vom Jahre 1846-1860 selbstandig erschienen sind. 2 vol. Leipzig, W. Engelmann, 1861. 
J'ai suivi la méthode généralement adoptée par les bibliographes, et qui consiste ì indiquer les différents travaux d'après l'ordre alphabétique des noms des auteurs, en ajoutant leurs prénoms, afin d'éviter les confusions qui pourraient résulter de la similitude des noms, et en joignant à cette énumération trois tables destinées à faciliter quelques recherches particulières. De plus, j'ai mis entre crochets les dénominations latines correspondant à certaines expressions étrangères, telles que Fire-Fly, Glow-Worm, Johanniswürmchen, Johanniswürmlein, Leuchtliafer, Spring-

Otto Taschenberg. - Bibliotheca zoologica, 1I. Verzeichniss der Schriften über Zoologie welche in den periodischen Werken enthalten und rom Jahre 1861-1880 selbstandig erschisnen sind. Leipzig, W. Engelmann, 1886. (En cours de publication).

Hermann-August Hagen. - Bibliotheca entomologica. Die Litteratur über das ganze Gebict der Entomologie bis zum Jalıre 186\%. 2 vol. Leipzig, W. Engelmann, 1862 et 1863 .

Gemminger et B. de Harold. - Catalogus Coleopterorum hucusque descriptorum synonymicus et systematicus. (Connu sous le nom de Catalogue de Munich). 12 vol. Munich, E.-H. Gummi (G. Beck), et Th. Ackermann, 1869-1876.

Bericht über die wissenschaftlichen Leistungen im Gebiete der Entomologie (anu. 1838-1885); recueil annuel rédigé par Wilhelm-Ferdinand Erichson, par Hermann-Rudolph Schaum, par Carl-Eduard-Adolph Gerstaecker, par l'riedrich Brauer, par Eduard-Larl von Martens, et par Philipp Bertkau. Berliu, Nicolaische Verlags-Buchhandlung, 1840-1886.

The Record of zoological Literature (ann. 1864-1869), continué sous le titre de The zoological Record (ann. 1870-1885). Londres, J. van Voorst, 1863 - 1886.

Et Zoologischer Jahresbericht herausgegeben von der zooloyischen Station zu Neapel. - Arthropoda (ann. 1880-1885); recueil annuel rédigé par Julius-Victor Carus, par Paul Mayer, et par Wilhelm Giesbrecht. Leipzig, W. Engelmann, 1881-1884, et Berlin, R. Friedlander et Sohn, 18850 - 1886 . 
kafer, etc., qui ne sont pas connues de tous les naturalistes, et j'ai donné quelques renseignements sur la nature du sujet traité et sur les Insectes étudiés dans les travaux dont le titre n'était pas par lui-même suffisannent explicite. Inutile d'ajouter que les noms employés par moi sont ceux dont les entomologistes se servent actuellement, et que j'ai remplacé les dénominations anciennes d'Elater noctilucus, L., de Lampyris ilalica, L.. etc., etc., par les expressions beaucoup plus correctes de Pyrophorus noctilucus, L. et de Luciola italica, L. Dans cette bibliographie, j’ai indiqué en français, non-seulement les titres des travaux, notes et observations qui figurent dans les recueils étrangers sans titre précis, mais aussi l’indication de quelques travaux dont je n’ai pu me procurer le titre véritable. Enfin, j'ai clonné, aussi exactement et aussi clairement que possible, les titres des recueils dans lesquels les différents mémoires ont été publiés, en mettant entre crochets les titres des publications qui ne donnent de ces mémoires qu'un extrait ou qu'une analyse, et en y joignant les indications absolument nécessaires de la date de publication des ouvrages, des numéros du tome, de la page, des planches et des figures, etc.

Relativement aux ourrages bibliographiques, je tiens à faire ici quelques observations critiques sur la façon dont certains bibliographes, sous le prétexte d'ètre concis, indiquent les recueils dlans lesquels sont insérés les travaux qu'ils mentionnent.

A cet égard, je ne citerai qu'un exemple, qui suffira, je crois, à me faire bien comprendre.

Dans une bibliographie concernant les Myriopodes, fort utile d'ailleurs, et qui émane d'un entomologiste disting'ué, le Bulletin de la Société des Amis des Sciences naturelles 
de Rouen est indiqué comme suit, à propos d'une variété nouvelle du Iulus longabo, C. Koch décrite par Robert Latzel : "Bull. Soc. Rouen, 1883, 271 " '. Il n’est pas difficile de voir combien ce renseignement est insuffisant. Je suppose, en effet, qu'un auteur ait besoin de se procurer le recueil en question. Quel bulletin devra-t-il demander, puisqu'il ne connaît même pas le nom de la Société qui le publie? Il sera donc obligé de se renseigner s'il existe à Rouen plusieurs Sociétés s'occupant de sciences naturelles, et de s'informer ensuite s'il n’a pas été publié dans le bulletin de 1883, à la page $27 \mathrm{I}$ du tome qui lui est inconnu, un mémoire sur les Myriopodes. On comprend de suite combien ces recherches seront fastidieuses, longues, dispendieuses même. Et cependant, il eut suffit de quelques lettres de plus pour les éviter entièrement. Au lieu de "Bull. Soc. Rouen, I883, 27I " l'auteur n'avait qu'à mettre "Bull. Soc. Amis Scienc. natur. de Rouen, $2^{\mathrm{e}}$ sem. I883, p. 271 ”, et toute recherche devenait inutile, le doute n'existant plus sur le nom de la Société et sur le nuniéro du bulletin à consulter. C'est une grave erreur, commise par beaucoup de savants actuels, de croire que tous ceux qui s'occupent d'histoire naturelle doivent forcément savoir que C.R. et S. N., pour n'en citer que deux exemples, sont l'indication abrégée des Comptes rendus hebdomadaires des séances de l'Académie des Sciences de Paris et du Systema Naturue, de Linné. Les bibliographies ont pour but essentiel de faciliter les recherches; elles doivent donc être rédi-

1. Alfred Preudhomme de Borre. - Tentamen Catalogi Lysiopetalidarun, Julidarum; Archiulidarum, Polyzonidarum atque Siphonophoridarum hucusque descriptarum, in Annal. de la soc. entomol. de Belgique, ann. 1884, t. XXVIII, p. 56 . 
gées avec la plus grande clarté possible, d'autant plus qu'elles s'adressent, non pas toujours à des savants de profession, mais encore, et souvent même, à des débutants dans les sciences. Je crois utile de signaler ces abus, qu'un amour exagéré de la concision ne saurait justifier, et j'espère que des réclamations réitérées finiront un jour par les faire entièrement disparaitre.

Un dernier mot encore avant d'abandonner cette question. Dans quelques-unes des nombreuses bibliographies que j'ai consultées, j'ai remarqué que leurs auteurs n'indiquaient pas le nom de la ville oú étaient publiés les recueils qu'ils citaient. C'est une omission fàcheuse, car il existe beaucoup de Sociétés dont les titres ne renferment pas le nom de la ville où elles siègent, et, dans ce cas, cette omission peut causer parfois des recherches assez longues.

Pour rédiger ma bibliographie des Insectes phosphorescents, j'ai dù forcément consulter un grand nombre d'ouvrages bibliographiques, de mémoires, de bulletins, d'annales et de recueils de toutes sortes, soit dans ma bibliothèque personnelle, soit dans celle des Sociétés savantes ve Rouen, soit, surtout, dans la riche Bibliothèque du Muséum d'Histoire naturelle de Paris, dont l'organisation et l'obligeance des Bibliothécaires sont au-dessus de tout éloge; mais je n'ai malheureusement pu vérifier par moi-même les titres de tous les travaux indiqués dans ma bibliographie, plusieurs d'entre eux étant presque introuvables aujourd'hui, ce qui explique pourquoi certains renseignements ne sont pas aussi complets que je l'eusse désiré.

Les travaux publiés sur les Insectes phosphorescents sont extrêmement nombreux, car ces animaux ont excité de tout temps la sagacité des naturalistes et l'imagination 
les littérateurs; mais je n'ai voulu citer que les travaux et les communications qui contenaient des observations plus ou moins originales, des notes utiles, ou des renseignements généraux sur la phosphorescence des Insectes, laissant complètement de côté tous les renseignements et observations puisés aux sources originales, qui figurent dans les traités classiques de Zoologie et de Physiologie ou dans les ouvrages le vulgarisation. De plus, je n'ai pas fait mention de quelques renseignements et indications bibliographiques relatifs à des Insectes phosphorescents, renseignements incomplets ou douteux, que j'ai trouvés dans certains ouvrages et dont il m’a été impossible de vérifier l'exactitude. J'ajouterai que dans ma bibliographie figurent comme étant lumineux différents Insectes qui ne le sont nullement en réalité; les phénomènes de phosphorescence que l'on a cru olsserver chez eux résultant d'observations fausses ou mal interprétées. Je devais, pour être complet, indiquer ces observations, au sujet desquelles j’ai donné des renseignements explicatifs dans mes Notes complémentaires. Enfin, dans le but de faciliter certaines recherches, j'ai joint à la table alphabétique des auteurs deux autres tables indiquant les noms des auteurs par groupe d'Inscetes lumineux et par date de publication de leurs travaux.

Malgré tout le soin que j'ai apporté dans la rédaction de cette bibliographie et dans la correction des épreuves, il est à peu près certain, hélas, que j'aurai commis des oublis et des erreurs provenant de l'insutfisance forcée de mes recherches et de mes vérifications; mais je suis certain d'être excusé, au moins par ceux qui ont entrepris de semblables travaux et qui en connaissent les multiples difficultés, sans parler du tenıps énorme qu'exigent ces ingrates 
recherches. J'ai cependant la conviction que le naturaliste qui voudrait se donner la peine de parcourir tous les ouvrages cités, aurait une idée suffisamment exacte de ce qui a été publié jusqu'à ce jour, au point de vue scientifique, sur les Insectes phosphorescents. 


\section{BIBLIOGRAPHIE GÉNÉRALE}

DES

\section{INSECTES PHOSPHORESCENTS}

(ANATOMIE, PHYSIOLOGIE ET BIOLOGIE)

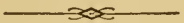

A

Afzelius, Adan. - Observations on the genus Pausus and description of a new species (Pausus sphaerocerus, Afz.), in Transact. of the linn. Soc., Londres, ann. 1798, t. IV, p. 243, et pl. XXII (P. sphaerocerus, fig. 1-6; $P$. microcephalus, fig. 1-5). [Coléoptères de la famille des Pausides].

Analyse in Wiedemann, C.-R.-W. - Archiv für Zoologie und Zootomie, Brunswick, ann. 1800, t. I, part. 2, p. 294; et in Annual Register, ann. 1805, p. 824.

Aldrovande, Ulysse. - De Animalibus Insectis libr septem. Bononiae, apud I.-B. Bellagambam, 1602. [Pyrophorus, p. 491; Lampyris, p. 493, av. fig. (p. 495)].

Allex, Benjamin. - Natural History of the mineral IIaters of Great Britain with observations on the Glow-Worm. Londres, 1711. [Lampyris noctiluca, L.].

Allman, George-James. - On the emission of light by Anurophorus fimetarius, Nicolet. in Proceed. of the 
roy. irish. Acad., Dublin, ann. 1850, t. V, p. 125. [Orthoptère du sous-ordre des Thysanoures et de la famille des Podurides].

Anghiera, Pietro-Martire D'. - Decades of the New-World.

- De rebus Oceanio et orbe novo Decades. Paris, 1536. [Observat. sur des Insectes phosphorescents].

Anonyme. - Natural Philosophy. Londres, Baldwin, IS29, p. 31. [Lampyres].

Axonyme. - The possibility of introducing and naturalising that beautiful Insect the Fire-Fly, in Magaz. of Nat. Hist., Londres, ann. 1832, p. 672. [Pyrophorus].

Axonyme. - Ueber das Betragen der Larven von Lampyris, in Isis, Encyklop. Zeitschr., Leipzig, ann. 1834, p. 850 ; et in Froriep, L.-F. vox. - Notizen aus dem Gebiete der Natur- und Heilkunde, Weimar, t. XIII, p. 321 .

Aristote. - Histoire des animaux. Traduction française par Cauus. Paris, Desaint, 2 vol., 1783, t. I, liv. Iv, chap. 1, p. 171. [Lampyrides].

Arnold, Carl. - Beitraege zur vergleichenden Physiologie, in Mittheil. der Naturforsch. Gesellsch. in Bern, ann. 1831, n 979-1003, p. 151. [Observat. sur la phosphorescence du Lampyre à ses différents états].

[Analyse in Mayer, P. et Giesbrecht, W.-Zoologisch. Jahresbericht der zoologisch. Station zu Neapel, Arthropoda, Berlin, ann. 1885, p. 138].

Aubert et Dubors, Raphaël. - Sur les propriétés de la lumière des Pyrophores, in Compt. rend. hebd. des 
séanc. de l'Acad. des Scienc., Paris, ann. IS84, t. XCIX, (séance du 15 septembre 1884), p. $47 \%$.

[Analyse in Mayer, P. et Giesbrecht, W. - Zoologisch. Jahreshericht der zoologisch. Station zu Neapel, Arthropoda, Berlin, ann. IS84, p. I64; et in The Journ. of Science and Annal. of Astronomy, Biology, etc., Londres, ann. 1884, p. 595].

Audoun, Jean-Victor.-Remarques sur la phosphorescence de quelques animaux articulés, à l'occasion d'une lettre de Forester sur la phosphorescence des Lombrics lerrestres, in Comp. rend. hebd. des séanc. de l'Acad. des Scienc., Paris, ann. 1810, t. XI, (séance du 9 novembre 18 10), p. 747. [Observat. sur le Lampyris noctiluca, L., p. 749].

Austin et Martin. - Notes sur la phosphorescence des Lampyrides, in Report of the entomol. Soc. of the Province of Ontario, ann. 1880, p. 17.

Auzoux, Hector. - Voir Tromelin.

Azara, Félix dE. - Voyages dans l'Amérique méridionale (1781-1801). Paris, Dentu, 1809, 4 vol. et 1 atlas, t. I, p. 111 et 211. [Observat. sur des Insectes phosphorescents].

\section{B}

BACH, Michael. - Ueber das Leuchten des Johanniswürmchen und einiger andern Insecten, in Natur und Offenbarung, Munster, ann. 1858, t. IV, p. 368. [Lampyris noctiluca, L.]. 
Bacon, François. - Sylva sylvarum, or a Natural History, published after the author's death by W. RAwLEY. Londres, 1627, p. 120 et 748. [Observat. sur des Insectes phosphorescents].

Bacourı, A. DE. - Osservazioni sulle Lucciole maggiori (Lampyris noctiluca, L.), 1785?

Baron. - Note sur la biologie du Pyrophorus noctilucus, L., in Annal. de la Soc. entomol. de France, Paris, ann. 1873, bull. des séanc., p. cxux.

BarRÈRe. - Essais sur l'Histoire naturelle de la France équinoxiale. 1834, p. 207. [Observat. sur des Insectes phosphorescents].

Bartholis, Thomas. - De luce animalium, lib. III. Lugduni Batavorum, ex officina Francisi Hackii, 1617, p. 205. [Observat. sur des Insectes phosphorescents].

Bates, Henry-IVilliam. - Sur la non-phosphorescence du Fulgora laternaria, L., in Transact. of the entomol. Soc. of London, ann. 1864, proceed., p. xir. [Cet auteur dit que, personnellement, il n’a constaté aucun phénomène de phosphorescence chez cet Hémiptère, et que les indigènes de l'Amazone, qui cependant connaissent bien cet Insecte et le considèrent comme venimeux, ne lui ont jamais parlé de sa luminosité].

[Analyse in Gerstaecker, C.-E.-A. - Bericht über Entomol., Berlin, ann. 1863-1864, p. 61].

Bates. - Voir Clark.

BEach, Alfred-E. [Editeur]. - The Science Record for 1874. A compendium of scientific progress and discorery 


\section{Z.P METK}

during the past year. New-York, 1875. [Observat. sur des Insectes phosphorescents].

Beauvols, Palisot de. - Voir Palisot.

BeCKer, J. von. - Ueber das Leuchtorgan der Lampyris splendidula, L., in Oefvers af Finska Vetensk.Soc. Forhandling., Helsingfors, ann. 1865-1866, t. VIII, p. 15.

Becker, Johann-Joseph-Uaria.-Le Fulgora laternaria, L. et sa Larve ne sont point lumineux d'après les observations de Beske, in Annal. de la Soc. entomol. de France, Paris, ann. 1818, bull. des séanc., p. xiv.

Beckerhein. - Observations sur la phosphorescence des Insectes, in Annal. de Chimie, Paris, ann. 1789, t. IV, p. 19.

Bellesme, Jousset de. - Voir Jousset.

Belon, Marie-Joseph. - Voir Trumelin.

BerNoului, Christoph. - Ueber das Leuchten des Meeres. mit besonderer Hinsicht auf das Leuchten thierischer Korper. Gottingue, Dieterich, 1803. [Observat. sur les Lampyres].

Berthold, Arnold-Adolph. - Lehrbuch der Physiologie. 1S29, t. I, p. 78. [Observat. sur les Lampyres, etc.]

Beske. - Voir Becker.

Béthune, C.-J.-S. - Sur une Larve lumineuse de Lampyride? ou d'Elatéride?, du Canada, in The canad. 
Entomol., Montréal, ann. 1863, p. 2. [Observat. sur cette larve par Morris, Couper et Osten-Sacken, p. 14 et 38 ].

Blanchard, Emile. - Voir Pastedr.

Blanchet, Rodolphe. - De la production de la lumière chez les Lampyres, in Biblioth. univers. des Scienc., Belles-Lettres et Arts, Genève, ann. 1856, t. XXXI, p. 213. [Analyse in Gerstaecker, C.-E.-A. - Bericht über Entomol., Berlin, ann. 1856, p. 79].

Blesson, L. - Observalions on the Ignis fatuus, in The entomol. Magaz., Londres, $\mathrm{n}^{\circ}$ 4, juillet 1 (\$33, p. 353. [Observat. sur les Lampyres?].

Borsduval, Jean-Alphonse. - De la phosphorescence des Chenilles de Mamestra oleracea, L., in Silbermaxx, G. - Revue entomol., Strasbourg et Paris, ann. 1833, t. I, p. 226. [Lépidoptère de la famille des Hadénides].

Borsduval, Jean-Alphonse. - Voir Tromelin.

Boll, Ernst-Friedrich-August. - Elater noctilucus, L. in Hamburg, etc., in Archiv des Vereins der Freunde der Naturgesch. in Necklenburg, Neubrandenburg, ann. 1857, $11^{\mathrm{e}}$ cah., p. 151. [Pyrophorus noctilucus, L.].

Bondaroy, Auguste-Denis, Fougeroux de. - Mémoire sur un Insecte de Cayenne, appelé Laréchal, et sur la lumière qu'il donne, in Mém. de l'Acad. des Scienc., Paris, ann. 1766, Mém. de l'Acad., p. 339; Hist. de l'Acad., p. 29, avec 1 pl.; et in Recens. Comment. Lips., t. XVIII, p. 73. [Pyrophorus noctilucus, L.]. 
Bonpland, Aimé. - Voir Humboldt .

Botroni, Dominique. - Pyrologia Typographiae. Naples, Parrini, 1692. [Observat. sur les Lampyres].

[Extrait in Frankische Acta Eruditor., Nuremberg, Suppl. t. XI, p. 1S8].

Bowles, George-H. - On luminous Insects, in Report of the entomol. Soc. of the Province of Ontario, ann. 1882, p. 34, et fig. 16. [Pyrophorus].

Bowring J.-C. - On the phosphorescence of Fulgora canclelaria, L., in Annal. and Nagaz. of Nat. Hist., Londres, ann. 1814, p. 127. [Cet auteur n'a pas observé la phosphorescence des Hotinus candelarius, L., à l'état libre ou en captivité].

Bravere, J.-C. - The repulation of the Lantern-Fly, in The americ. Naturalist, Philadelphie, ann. 1885, 1. XIX. p. 834, av. fig. [Cet auteur parle des idées fausses qui ont cours au Brésil sur le Fulgora laternaria, L.. appelé " Jitirana Boïa », et nie que cet Hémiptère soit phosphorescent et venimeux].

Brown, Patrick. - The civil and natural History of Jamaïca. Londres, 1756, p. 431. [Observat. sur des Insectes phosphorescents].

Brown, Patrick. - Voir Tiedenann.

Brugnatelli, Luigi-Gaspar. - Annali di Chimica e Storia naturale, etc. Pavie, ann. 1790-1802, 21 vol., t. XIII (1797). [Observat. sur la phosphorescence des Lampyres, etc.]. 
Brugnatell, Luigi-Gaspar. - Nota sul fosforismo di vari corpi e segnalamente degli animali vivi, in Giorn. di Fisica, Chimica e Storia naturale, etc. Pavie, ann. 1814, t. VII, p. 238. [? Observat. sur des Insectes phosphorescents].

Bruguière, Jean-Guillaume. - Observations sur les Lampyres, in Journ. d'Hist. natur., Paris, ann. 1792, t. II. p. $26 \%$.

Brullé, Auguste. - Voir laporte.

Burmeister, Hermann-Carl-Conrad. - Handbuch der Entomologie, Berlin, t. I (1832), p. 535. [Généralités sur les Insectes phosphorescents, avec de nombreux renseignements bibliographiques].

Burneister, Hermann-Carl-Conrad. - Observations on a light-giving Coleopterous Larva, from Parand, in The Journ. of the linn. Soc. of London, Zoology, ann. 1871, p. 416, av. fig. [Larve inconnue d'un Coléoptère] .

Burnetr, Waldo-Irvin. - On the luminous spots of the great Fire-Fly of Cuba (Pyrophorus phosphoreus), in Proceed. of the Boston soc. of Nat. Hist.. ann. 1850. t. III, p. 290 .

\section{C}

Canerarius, Johann-Rudolph. - Cicindelae historia, fulgoris calculo vesicae frangendo utilis, in Syllog. memorab., 1621, Cent. 4, part. 30, p. 208; Cent. 19. part. 37, p. 1541. [Lampyres?]. 
Cameron, John. - Our tropical Possessions in malayan India, p. 80. [Observat. sur des Insectes phosphorescents].

[Analyse in The Zoologist, Londres, ann. I865, t. XXIII, p. 9739].

Candèze, E. - Histoire des métamorphoses de quelques Coléoptères exotiques, in Ném. de la Soc. roy. des. Scienc. de Liége, ann. I86I, t. XVI, p. 36I, et pl. III, fig. 5. [Description de la larve du Photuris pensylvanica, Degeer].

Candèze, E. - Voir Suitu.

Carpenter, William-Benjamin. - The popular Cyclopaedia of Natural Science. - Animal Physiology. Londres, Orr et $\mathrm{C}^{\circ}, 1843$. [Pyrophores et Lampyrides, p. 397; Fulgorides, p. 400 et 679$]$.

Carradori, Giovacchino. - Expériences et Observations sur la phosphorescence des Lucioles (Lampyris ilalica, L.), in Annal. di Chimica e Storia naturale, etc., Pavie, ann. I797, t. XXVI, p. 96; in The philosoph. Magaz., Londres, ann. I798, t. II, p. 77 ; et in Volgt, J.-H. - Magaz. für das Neuest. aus der Physik und Naturgesch., Gotha, ann. I799, t. I, part. 4, p. I29. [Luciola italica, L.].

Carradori, Giovacchino. - Esperienze ed Osservazioni sopra il fosforo delle Lucciole (Lampyris ilalica, L.), in Giorn. di Fisica, Chimica e Storia naturale, etc., Pavie, ann. Is08, t. I, p. 269. [Luciola italica, L.]. 
Carradori, Giovacchino. - Dell eccitabilità del fosforo delle Lucciole (Lampyris italica, L.), in Giorn. di Fisica, Chimica e Storia naturale, etc., Pavie, ann. 1814, t. VII, p. 306. [Luciola italica, L.].

Carrara, Marcellino. - Sulla phosphorenza della Lucciola comune (Lampyris italica, L.), in Biblioth. italiana, Milan, ann. 1836, t. LXXXII, p. 357, av. 1 pl. [Luciola italica, L.].

[Analyse in L'Institut, Paris, ann. 1836, t. IV, $\mathrm{n}^{\circ}$ 188, p. 424].

Carus, Carl-Gustav. - Ueber das Licht der italienischen Leuchtkafer, in Carus, C.-G.-Analecten zur Naturund Heilkunde, Leipzig, 1829, p. 169. [Luciola italica, L.].

Carus, Carl-Gustav. - Expériences sur la matière phosphorescente de la Lampyris italica. L.; action de l'eau pour rendre à la matière desséchée cette phosphorescence ; (extrait d'une lettre adressée par l'auteur), in Compt. rend. hebd. des séanc. de l'Acad. des Scienc., Paris, ann. 1864. t. LIX, (séance du 10 octobre 1864), p. 607. [Luciola italica, L.].

Castelnau, de. - Voir laporte.

Chabrillac, François. - Sur la non-phosphorescence du Fulgora laternaria, L., in Annal. de la Soc. entomol, de France, Paris, ann. 1859, bull. des séanc., p. cir.

Charpentier, Toussalnt de. - Horae entomologicae. Wratislaviae, Gosohorsky, 1825, p. 192, et pl. VI, fig. 5-6. [Description de la larve de la Luciola italica, L.]. 
Champion, George-C. - Biologie des Fulgorides, in Transact. of the entomol. Soc. of London, ann. 1883, proceed., p. xx. [D'après cet auteur, les Fulgorides ne sont pas phosphorescents].

Chevrolat, Auguste. - Description du genie Dadoychus, in Silbernans, G. - Revue entomol., Strasbourg et Paris, ann. 1833, t. I, descript. $\mathrm{n}^{\circ} 1$ 1, p. 4, av. fig. col. [Coléoptères de la famille des Cérambycides].

Chevrolat, Auguste. - Voir Tronelin.

Clark, Hanlet, Mac Lachlan, Bates, Saunders, etc. Discussion sur la phosphorescence des Insectes, in Transact. of the entomol. Soc. of London, ann. 1865, proceed., p. LxLIV .

Columna, Fabius-Lincaeus. - Aquatilium et terrestrium aliquot animalium aliorumque naturalium rerum observationes. Rome, Mascardi, 1616, cap. XVII. [Lampyrides].

Conroy et SpIller. - Spectre de la lumière du Lampyris noctiluca, L., in Nature, Londres et New-Yurk, 1882, t. XXVI, p. 319 et 343.

COUPER. - Voir BÉTHUne.

Curtis, John. - An account of Elater noctilucus, L., the Fire-Fly of the West-Indies, in The zoolog. Journ., Londres, ann. 1827, t. III, $\mathrm{n}^{\circ} \mathrm{xI}$, p. 379. [Pyrophorus noctilucus, L.].

[Extrait in FÉrussac, A. DE. - Bull. univers. des Scienc. natur. et de Géologie, Paris, ann. 1829, t. XVI, p. 327; in Heusinger, C.-F. - Zeitschr. für organ. 
Physik, Eisenach, ann. 1829, t. III, cah. I, p. 137; in Fronice, L.-F. vox. - Notizen aus dem Gebiete der Natur- und Heilkunde, Weimar, ann. 1829, t. XXIT, p. 344; ann. 1830, t. XXVIII, p. 1; in Tнох, T.-C.-G. Archiv der Naturgesch., Naumburg, ann. 1830, t. II, part. 2, p. 63; in Isis, Encyklop. Zeitschr., Leipzig, ann. 1830. t. NI, p. 1171].

\section{$\mathrm{D}$}

DaLE, James-Charles. - The Glow-Worm is plentiful in many parts of the Kingdom, in Magaz. of Nat. Hist., Londres, ann. 1834, p. 250. (Remarques à ce sujet par James Jexingas, James MaIN, Edward Wilsox et HughEdwin STRICKLAND). [Lampyris noctiluca, L.].

DaLE, James-Charles.-Note on the Glow-Worm, in Hagaz. of Nat. Hist., Londres, ann. 1834, p. 253. [Lampyris noctiluca, L.].

Darwix, Charles. - Voyage d'un Naturaliste autour du Monde, traduit de l'anglais par Edmond Barbier, Paris, C. Reinwald et $\mathrm{C}^{\mathrm{i} \text {, }}$, 1875. [Observat. sur des Insectes phosphorescents].

Daumost, G. - Sur une Nymphe de Lampyris noctiluca, L. présentant deux points très-lumineux, in Annal. de la soc. entumol. de France, Paris. ann. 1851, bull. des séanc., p. c11.

Davr, Humphry. - On the light of the Glow-Worm, in Philosoph. Transact. of the roy. Soc. of London, ann. 1810, p. 237. [Lampyris noctiluca, L.]. 
Degaerr ${ }^{1}$, Carl. - Lyckte-Masken fran China (Fulgora candelaria, L.), in Svenska Vetensk. Acarl. Handl., Stockholm, ann. 1746, t. VII, p. 65: Deutsche Uebers., ann. 1752 , t. VIII, p. 67 , av. fig.; et in Latein. Uebers., in Analecta transalpina, t. I, p. 475. [Ho imss comlelarius, L.]. [Voir LıNné].

DEgeer, Carl. - Mémoire sur un Ver-luisant femelle et sur sa transformation, in Mém. de l'Acad. des sicienc., Paris, Savants étrangers, ann. 1755, t. II, p. 261, et pl. IX, fig. 1-12. [Lampyris noctiluca, L.].

[Traduction in Goeze, J.-A.-E.-Karl Bonnet's wie auch einig. andern berühmt. Naturforsch. auserl. Abhandl. aus der Insectologie, etc. Halle, Gebauer, 1774, p. 348].

Degler, Carl. - Mémoires pour servir à l'Histoire des Insectes. 7 tom. (8 vol.), av. pl., Stockholm, L.-L. Grefing, et P. Hesselberg, 1752-1778. - T. III (1773), Fulgora laternaria, L., p. 195; Hotinus candelarius, L., p. 197. T. IV (1771), Lampyris noctiluca, L., p. 31, et pl. I, fig. 19-33; Luciola italica, L., p. 54, et pl. XVII, fig. 9-11; Pyrophorus noctilucus, L., p. 160, et pl. XVIII, fig. 1; Pyrophorus phosphoreus, L., p. 161, et pl. XVIII, fig. 2.

Desmarest, Eugène. - Voir Tromelin.

1. La veritable orthographe du nom de cet illustre naturalisle est Degeer et non de Geer. (Renseignement communiqué par Degeer lui-mème à l'entomologiste allemand I.-A.-E. Goeze et cité par ce dernier á la page xIV de lintrorluction à ses Entomologische Beitraege zu des Ritter Linné zwolften Ausgalje des Natursystems. Leipzig, Weidmann, 3 tom. (¿ vol.), $177 \pi-1781)$. 
Dieckнorf, Ludw.-A.-Ueber das Leuchten der LampyrisArten, in Stettiner entomol. Zeit., ann. 1842, t. IIl. p. 117.

Dollfus, Adrien. - Note sur la présence du Lamprohiza ${ }^{1}$ splendidula, L. dans les environs de Spa, in Feuille des Jeunes Naturalistes, Paris, $11^{\mathrm{e}}$ ann. (1881), $n^{\circ} 131$ ( $1^{\text {er }}$ septembre 1881 ), p. 152.

Dortous de Mlaran, Jean-Jacques. - Dissertation sur la cause de la lumière des Phosphores et des Noctiluques. Paris, 1715, ou Bordeaux, R. Lebrun, 1i17. p. 54. [Observat. sur des Insectes phosphorescents].

Doubledar, Edward. - Discussion on the Luminosity of Fulgora candelaria, L., in The entomol. Magaz., Londres, ann. 1836, t. III, p. 45 et 105. [Hotimus candelarius, L.] .

Dubors, Raphaël et Regnard, Paul. - Note sur l'action des hautes pressionis sur la fonction photogénique du Lampyre, in Compt. rend. hebd. des séanc. de la Soc. de Biologie, Paris, ann. 1884, p. 675. [Lampyris noctiluca, L.].

Dubors, Raphaël. - Sur la lumière des Pyrophores, in Compt. rend. hebd. des séanc. de la Soc. de Biologie, Paris, ann. 1884, n ${ }^{\circ} 37$.

1. Lamprohiza, et non Lamprorhiza, comme l'écrivent la plupart des

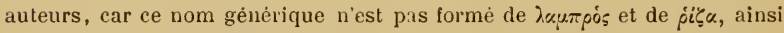
que l'indique le Catalogue de Munich, mais bien de iaukpos et de isw. [Cf.-Jules Bourgeuis. - Faune gallo-rhenane. Malacodermes. Lampyrini, p. 77 (en note). [ Publièe dans la Revue d'Entom logie, Caen]. 
Dubors, Raphä̈l. - Nute sur la physiologie des Pyrophores, in Compt. rend. hebd. des séanc. de la Soc. de Biologie, Paris, ann. 188 1, n 40, p. 661 .

I) bors, Raphaël. - Fonction photogénique des Pyrophores, in Compt. rend. hebd. des séanc. de la Soc. de Biologie, Paris, ann. 1885, n 30, p. 553.

Dubors, Raphaël. - Contribution à l'étude de la pro. duction de la lumière par les êtres vivants. - Les Elatérides lumineux, in Bull. de la Soc. zoolog. de France, Paris, ann. 1886, p. 1, et pl. I-IX, avec fig. dans le texte, et un buste de Claude Bernard, photographié par la lumière animale. - Tirage à part, Mleulan, 1886. [Ce travail, qui a servi de thèse à l'auteur pour obtenir le grade de Docteur ès-sciences naturelles, est une œuvre de premier ordre, qui éclaire considérablement cette importante question physiologique].

Dubors, Raphaël. - De la fonction photogénique dans les OEufs clu Lampyre, in Bull. de la Soc. zoolog. de France, Paris, ann. 1887 , fasc. 1 .

Dubors, Raphaël. - Voir Aubert.

Dufour, Léon. - Recherches anatomiques sur les Carabiques et sur plusieurs autres Coléoptères, in Annal. des Scienc. natur., Paris, ann. 1824, t. III, p. 225, etc. [Anatomie du Lampyris noctiluca, L.].

Dutertre. - Histoire générale des Antilles francaises. Paris, 1667, p. 280. [Observat. sur des Insectes phosphorescents]. 


\section{E}

Eaton, A.-E. - Notes on the intermittent light of Luciolu lusitanica, Charp., in The Entomol. monthl. Magaz., Londres, ann. 1880, t. XVII, p. 94.

Eaton, A.-E. - Sur la phosphorescence du Caenis dimidiata, Steph., in Transact. of the entomol. Soc. of London, ann. 1880, proceed., p. vin. [Orthoptère pseudoNeuroptère de la famille des Ephémérides].

Eatox, A.-E. - Luminous May-Fly from Ceylon, in Transact. of the entomol. Soc. of London, ann. 1882, proceed., p. xur. [Cet Insecte est probablement le Teloganodes tristis, Hag., ơ, Orthoptère pseudo-Neuroptère de la famille des Ephémérides. - Observat. à ce sujet par George Lewis et Charles-Owen Waterhouse]. Eaton, A.-E. - Voir Hagen.

Edwards, H. - Sur la phosphorescence du Fulgora laternaria, L., in Transact. of the entomol. Soc. of London, ann. 1818, proceed., p. xxxvili. [Phosphorescence arlmise d'après les assertions de quelques indigènes]. Edwards, Henri MiLNe. - Voir Milne-Edwards.

Ehrenberg, Christian-Gottfried. - Das Leuchten des Meeres. Neue Beobachtungen nebst Uebersicht der. geschichtlichen Entwiclilung dieses merliwürdigen Phaenomens, in Abhandl. der koniglich. Akad. der Wissenschaft. zu Berlin, ann. 1834, p. 411, av. 2 pl. [Dans le chapitre intitulé : Geschichtliche Uebersicht der Beobachtungen und Erklarungen des Meeresleuchtens (p. 413-525), cet auteur indique et analyse sommairement tous les mémoires, notes, observations, etc., relatifs à la phosphorescence des animaux et des 
végétaux, qui ont été publiés jusqu'en 1834. Ehrenberg donne aussi (p. 519) des détails sur la structure des organes phosphorescents des Pyrophores et des Lampyres].

EMERy, Carlo. - Studi intorno alla Luciola italica, L., in Bull. della Soc. entomol. italiana, Florence, $15^{\mathrm{e}}$ ann. (1883), p. 32\%. [Résumé du travail suivant].

EMery, Carlo. - Untersuchungen über Luciola italica, L., in Zeitschr. für wissenschaftl. Zoologie, Leipzig, ann. 1S84, t. XL, p. 338, et pl. XIX, fig. 1-21. [Travail très-important]. [Résumé de l'auteur in Archiv. italien. de Biologie, Turin, ann. 1884, t. V, p. 175, av. 2 fig.]. [Analyse in Mayer, P. et Giesbrecht, IV. - Zoologisch. Jahresbericht der zoologisch. Station zu Neapel, Arthropoda, Berlin, ann. IS81, p. 165; in Bertkau, P. et Martens, E. yon. - Bericht über Entomol., Berlin, ann. 1881, p. 211; et in The Journ. of Science and Annal. of Astronomy, Biology, etc., Londres, ann. 1S84, p. 5991 .

Enery, Carlo. - La luce della Luciola italica, L. osservata col microscopio, in Bull. della soc. entomol. italiana, Florence, $17^{\mathrm{e}}$ ann. (1S55), p..351, et pl. V; in Archiv des Scienc. physiq. et natur., Genève, ann. 1855, t. XIV, p. 272 ; et in Archiv. italien. de Biologie, Turin, ann. 1886, t. VIl, $2^{\circ}$ fasc.

[Analyse in Mayer, P. et Giesbrecht, IV. - Zoologisch. Jahresbericht der zoologisch. Station zu Neapel, Arthropola, Berlin, ann. 1855, p. I18; et in Bertkau, P. Bericht über Entomol., Berlin, ann. 1855, p. 287]. 
Eмmert. - Aehnlichlieit aufmerksam welche swischen den Tracheensellen im Leuchtorgan von Lampyris und den Langerhuns'schen, in Schultze, M.-S. Archiv für mikroskop. Anatomie, Bonn, ann. 1872, t. VIII, p. 652.

[Analyse in Bertkau, P. - Bericht über Entomol., Berlin, ann. 1873-1874, p. 49 (301)].

Evell, Henrik.-Fosforescensen hos Lysmasken, in Entomologisk Tidskrift, stockholm, ann. 1881, t. I, $2^{\circ}$ cah., p. 101. [Phosphorescence du Lampyris noctiluca, L.. - écrit en langue suédoise]. [Résumé in même Bull. : Sur la phosphorescence du Ver luisant (Lampyris noctiluca, L.), p. 117].

Erichson, Wilhelm-Ferdinand. - of Pyrophorus from Cuba, in Wieguavs, A.-F.-A. - Archiv für Naturgesch., Berlin, ann. 1841, t. VII, p. 87.

Erichson, Wilhelm-Ferdinand. - Zur systematischen Kenntniss der Insectenlarven, in Wiegmans, A.-F.-A. - Archir für Naturgesch., Berlin, ann. 1841, t. VII, p. 90. [Généralités sur les larves de Lampyrides].

Evass, W.-T. - Note sur la phosphorescence du Fulgora laternaria, L., in Transact. of the entomol. Soc. of London, ann. 1865, proceed., p. cir. [Observat. personnelle de la phosphorescence de cet Hémiptère].

\section{$\mathrm{F}$}

FaILle, Johann-Marcus-Baart DE LA. - De animalibus phosphorescentibus. Dissert. inaugur., Groningue, Bolt, 1\$21. [Observat. sur des Insectes phosphorescents]. 
Fairmaire, Léon. - Nolice sur les Coléoptires récoltés par J. LÉdÉrer sur le Bosz-Dagh (Asie-.Vineure), in Annal. de la Soc. entomol. de France, Paris, ann. 1866, p. 262. [Description de la larve du Lampyris maculicollis, Fairm.].

Fennell, James. - Localities near London in which the Glow-Worm has occuried, the Larva differs from the perfect Insect, the Eggs are luminous, in Magaz. of Nat. Hist., Londres, ann. 18:35, p. 625. [Lampyris noctiluca, L.].

Ferchault de Réaumur, René-Antoine. - Voir Réaumur.

Fermin, Philippe. - Histoire naturelle de la Hollande équinoxiale, ou Description des animaux, plantes, fruits, et antres curiosités nalurelles, qui se trouvent dans la colonie de Surinam. Amsterdam, Magérus, 1765. [Cet auteur prétend que le prolongement céphalique du Fulgora laternaria, L. est phosphorescent, p. 136].

Forster, Johann-Georg-Adam. - Ein Versuch mit dephlogistisirter Luft über das Leuchten von Lampyris splendidula, L. (en collaboration avec S.-T. von SoEuslerring), in Gotting. Magaz. der Wissenschaft. und Litteratur, Gottingue, ann. I783, part. 2, p. 281. [Lamprohisa splendidula, L.].

[Extrait in Journ. de Physique, Paris, ann. 1783, t. XXIII, p. 24; in Opuscoli scelti, ann. 1783, t. VI, p. 419 ; et in Fusscr, J.-C. - Neues Magaz. für die Liebh. der Entomol., Zurich et Winterthur, ann. 1784, t. 1I, p. 103].

Fougeroux de Bondaroy, Auguste-Denis. - Voir Bondaror. 
Foulques de Villaret. - Voir Villaret.

Fri, Mlexander. - Note sur la phosphorescence des Aspidosoma, in Transact. of the entomol. Soc. of London, ann. 1865, proceed., p. C. [Coléoptères de la tribu des Lampyrides].

\section{G}

Gadeau de Kerville, Henri. - Les Insectes phosphorescents. Rouen, Léon Deshays, 1881, av. 1 pl. chromolithographiées. [Ouvrage de vulgarisation].

Gaxdolphe, Paul. - Voir Tromelin.

Geer, Carl de. - Voir Degeer.

Grorge, H. jun. - Note sur la phosphorescence du Goërius olens, Müller, in Transact. of the entomol. Soc. of London, ann. 1851, proceed., p. cxr1l. [Stapleylinus olens, Müller; Coléoptère de la famille des Staphylinides. - Cet Insecte derait sans doute son apparence lumineuse à un corps phosphorescent quelconque qui adhérait à lui].

Gernez. - Voir Pasteur.

Ginnerthal, Benjamin-August. - Observalions sur la métamorphose de quelques Iiptères de la famille des Muscides, et sur la phosphorescence d'une c'henille de Noctuelle (Noctua occultu, L.), in Bull. de la Soc. impér. des Natural. de Moscou, ann. 1829, t. I, p. 136. |Agrotis occulta, L., Lépidoptère de la famille des Agrotides]. 
[Extrait in Férussac, A. DE. - Bull. univers. des Scienc. natur. et de Géologie, Paris, ann. 1831, t. XXVII, p. 101 ; et cité par Alexandre Lefebvre, in Annal. de la Soc. entomol. de France, Paris, ann. 1832, p. 121]. Grirard, Maurice. - Etulles sur la chuleur libre dégagée pur les animaux invertébrés et spéciulement les Insertes, av. 2 pl. Paris, V. Masson et fils, 1869. [Thèse pour obtenir le grade de Docteur ès-sciences naturelles]. [Observat. sur la chateur d'un Ver luisant, p. 9 ; et sur la chaleur du Lampyris noctiluca, L. et du Luciolu itulica, L., p. 12i].

Girard, Maurice. - Les Taupins lumineux, in La Nature, Paris, ann. 1873, (nº du $1^{\text {er }}$ novembre 1873), p. 337, av. fig. [Pyrophores et Photophores].

Grrard, Maurice.-Les Insecles. Traité élémentaire d'Entomoloyie. Paris, J.-B. Baillière et fils, 3 tom. (4 vol.), av. de nombr. pl., 1873-1885. - T. I. (1873), Pyrophorus et Photophorus, p. 510 et 512 ; Lampyris, Lamprohisa, Phosphaemus, Cratomorphus, Amythetes et Luciola, p. 522-530; Cratomorphus splendidus, I)rury, \&, pl. XXXV, fig. 7, 7 a, 7 b, 7 c et $7 \mathrm{~d}$; Amythetes (antennes), pl. XIIV, fig. 8. - T. IIl, $2^{\mathrm{e}}$ fasc. (1885), Fulgora et Hotimus, p. 857 et 858; Hotinus Lathburi, Kirby, pl. CII, fig. 2.

Glover, Townsend. - Voir Townsend.

Gonun, Henry-S. - Notes on the structure of Lampyriclae, in The Entom. monthl. Magaz., ann. 1880, t. NVI, p. 261.

Gorman, Henry-s. - Structure of the Lampyridae with reference to their phosphorescence, in Transact. of the 
entomol. Soc. of London, ann. 1880, p. 63. [Discussion, proceed., ann. 1880, p. vi].

Gosse, Philip-Henry. - On the Insects of Jamaïca, in Ann. and Magaz. of Nat. Hist., Londres, ann. 1848, p. 200. [Phosphorescence du Pyrophorus noctilucus, L.].

[Analyse in Schaum, H.-R. - Bericht über Entomol., Berlin, ann. 1848, p. 40].

Gosse, Philip-Henry.-Note sur le Lampyris noctiluca, L., in The Entomologist, Londres, ann. 1880, p. 20.

Gounelle, Emile. - Note sur la biologie du Fulgora laternaria, L., in Annal. de la Soc. entomol. de France, Paris, ann. 1886, bull. des séanc., p. c. [L'auteur n’a pas constaté la phosphorescence de cet Hémiptère].

Goureau. - Note sur l'Aspisoma candellaria, Reiche, in Annal. de la Soc. entomol. de France, Paris, ann. 1845, p. 345, et pl. VII (11, fig. 1-6). [Description de la larve de ce Lampyride, etc.].

Greenwood Penny, R. - Glow-Worms v. Snails, in Nature, Londres et New-York, 1879, t. XX, ( $\mathrm{n}^{\circ}$ du 3 juillet 1879), p. 220. [Lampyris noctiluca, L.].

Grew, Nehemiah. - Museum regalis societatis : or a catalogue and description of the natural and artificial rarities belonging to the Royal Society and preserved at Gresham College. Londres, Malthus, 1681, p. 158. [Note sur le Fulgora laternaria, L., dont le prolongement céphalique, d'après Grew, est phosphorescent. - Cet ouvrage renferme le premier dessin que l'on possède du Fulgora laternaria, L., 
désigné par Grew sous le nom de Cucujus peruvianus. Ce dessin a été fait d'après un exemplaire desséché].

Grosov, Lorenz-Theodor. - Zoophylacium Gronoviamum. Lugduni Batavorum, Haak, $2^{\circ}$ fasc. (1774), p. 152, $\mathrm{n}^{\circ} 474$. [Observat. sur des Insectes phosphorescents].

Grotthuss. - Observations sur la phosphorescence du Lamplyris íalica, L., in Annal. général. des Scienc. physiques, Bruxelles, ann. 1821, t. VIII, p. 31. [Luciola italica, L.] .

Guévead de Montbeillard, Philibert. - Mémoire sur la Lampire ou Ver luisant, in Nouv. Mém. de l'Acad. de Dijon, $2^{\text {me }}$ sem. 1782, p. 80. [Lampyris noctiluca, L.]. [Extrait in Beckmanx, J. - Physikalisch-oekonomische Biblioth., etc., Gottingue, t. XIV, p. 265].

Guenther, Johann. - Von allerhand Insecten, in Breslauer Natur- und liunstgeschichte, ann. I721, versuch 30 , p. 403. [Lampyres].

Gullding, Landsdown. - Notes on luminous Insects, chiefly of the West-Indies, on luminous Meteors, on Ignes fatui, etc., in Magaz. of Nat. Hist., Londres, an1. 1831, p. 579. [Observat. sur des Insectes phosphorescents].

\section{$\mathrm{H}$}

HaAse. - Zur Biologie der Kafergattung Phengodes, in Sitzungsberichte der naturwissenschaftl. Gesellsch. Isis in Dresden, ann. 1885, p. 10.

[Analyse in Bertiau, P. - Bericht über Entomol., Berlin, ann. 1885, p. 231]. 
Hagex, Hernann-Iugust. - Aelteste Nachricht über das Leuchten der Fulgora laternaria, L., in stettiner entomol. Zeit., ann. 1853, t. . IIT, p. 5.5. [Notice historique].

HAGEx, Hermann-August. - On the Luminosity of Fulgora laternaria, L., in The Entomol. monthl. Magaz., Londres, ann. I865, t. I, p. 250. [Notice historique].

[Analyse in Gerstaecher, C.-E.-A. - Bericht über Entomol., Berlin, ann. 1865-1866, $2^{\circ}$ cah., p. 114].

Hages, Hermann-August. - Notes on the Ephemeridae, compiled (with remarks) by 1 .-E. EATos, in Transact. of the entomol. Soc. of London, ann. 18\%3, p. 399. [Caenis dimidiata, Stẹph., o*, lumineux? (Orthoptère pseudo-Neuroptère de la famille des Ephémérides)].

HaMlet. - Voir Clark.

Haxcock John. - Note upon the Luminosity of Fulgora laternaria, L., in Transact. of the entomol. Soc. of London, ann. 1834, t. I, proceed., p. xxxı; in Proceed. of the zoolog. Soc. of London, ann. 1831, t. II, p. 19; in L'Institut, Paris, ann. 1831, p. 366 ; in Froriep, L.-F. von. - Notizen aus dem Gebiete der Natur- und Heilkunde, Weimar, amm. 1831. t. XLI, p. 341; et cité par Alexandre Lefebrre, in Annal. de la Soc. entomol. de France, Paris, ann. 1834, bull. des séanc., p. Lxıll. [Hancock nie la propriété phosphorescente de cet Hémiptère].

IIArting, Pieter. - Skizzen aus der Natur. Aus dem hollandischen übersetzt von J.-E.-A. Martix, Leipzig, W. Engelmann, 2 vol., 1854 et 185\%, t. I (1854), p. 59. [Observat. sur des Insectes phosphorescents]. 
Ilemamann, Carl. - Lntersuchungen über die Leuchtorgane der bei Vera-Crus vorkommenden Leuchtiafer (Pyropleorus sp.), in sicnultze, M.-S. - Mrchiv fül mikioskop. Anatomie, Bonn, ann. 1872, 1. VIII, p. 161 ; et in La Naturaleza, Periodico cientif. de la soc. mexicana de Hist. natur., Mexico, t. III (ann. 1874-1876), p. 10 et 54 .

[Analyse in Bertiau, P. - Bericht über Entomol., Berlin, ann. 1873-187:, p. 17 (:99)].

Heinemann, Carl. - Aschenanalyse von 186 Bauchleuchtorganen der bei Vera-Cruz vorkommenden Leuchtkafer (Pyrophorus sp.), in Pfluger, E.-F.-W. - Archir für die gesammte Physiologie des Menschen und der Thiere, Bonn, $\mathrm{S}^{\mathrm{e}}$ ann. (1854), p. 365; et in La Naturaleza, Periodico cientif. de la Soc. mexicana de Hist. natur., Mexico, t. III (ann. 1874-1876), p. 97.

Heinrich.-Die Phosphorescen a der Korper. $3^{\text {me }}$ Abhandl., Nuremberg, 1815, p. 375. [Observations sur le Lampyris noctiluca, L.].

Hebia. - Merliwürdige Beobachtungen vom Johanniswürmchen (Lampyris noctiluca, L.), in Volgt, J.-H. - Magaz. für den neuest. Zustand der Naturkunde, etc., Weimar, ann. 1805, t. IX, p. 166.

Hexderson, George.-Note on the Glow-Worm, in Transact. of the Berwickshire Natural. Club, Berwick, ann. I8 13, t. II, p. 98. [Lampyris noctiluca, L.].

Henning, Johann-Friedrich. - Von einigen Insectis oder Ungeziefer. - II. Von den Cicindelis oder Johannis- 
Würmlein, in Breslauer Natur- und Kunstgeschichte, ann. 1724, versuch 30, p. 515. [Lampyris noctiluca, L.]. Hexslow, George. - Frogs and Glow-Flies, in Nature, Londres et New-York, I879, t. AX, (nº du 3 juillet Is79), p. 220. [Lampyrides].

Hermanas, de Dos. - Sur les Cocuyos de Cuba, in Compt. rend. hebd. des séanc. de l'Acad. des Scienc., Paris, ann. I873, t. LXXVII, (séance du 4 août I873), p. 333. [Pyrophorus noctilucus, L.].

Hermbstaedt, Siegmund-Friedrich. - Bemerkungen über das Leuchten organischer Korper im Leben und nach dem Tode derselben, in Magaz. der Gesellsch. der Naturforsch. Freunde zu Berlin, ann. IS08, t. II, p. 248: [Observat. sur les Lampyres].

Heward, Robert. - Memorandum on the Fire-Flies of Jamaïca, in The Entomologist, Londres, ann. I841, p. 12. [Différence dans l'émission de la lumière chez les Pyrophores et les Lampyres].

Hoeven, Jan var der. - Eenige Woorden over het Lichten van den suid-amerikaanschen Springliever, in Album der Natuur, Haarlem, ann. I855, aflev. 7, p. 205. [Pyrophorus].

HoffmansegG, Johann-Centurius von. - Ueber das Leuchten von Fulgora, in Magaz. der Gesellsch. der Naturforsch. Freunde zu Berlin, ann. 1807, t. I, p. 152. [Hoffmansegg nie la propriété phosphorescente de cet Hémiptère]. [Extrait in? Philosoph. Transact. of the roy. Soc. of 
London, ann. 1807, p. 52; et in Isis, Encyklop. Zeitschr., Leipzig, ann. 1818, t. IX, p. 1453].

Hudson, G.-V.-A luminous Insect-Larva in New-Zealand, in The Entomol. monthl. Magaz., Londres, ann. 1886, t. XXIII, p. 99. [Larves d'un Diptère appartenant trèsprobablement à la fanille des Nycétophilides]. [Voir Osten-Sacken].

Hulme, Nathaniel.-Experiments and Observations on the Light which is spontaneously emitted, with some Degree of Permanency, from various Bodies, in Philosoph. Transact. of the roy. Soc. of London, ann. 1800, p. 161. [Expériences sur la phosphorescence du Lampyris noctiluca, L.].

Hulue, Nathaniel. - A Continuation of the Experiments and Observations on the Light which is spontaneously emitted from various Bodies, with some Experiments and Observations on solar Light when imbibed by Canton's Phosphorus, in Philosoph. Transact. of the roy. Soc. of London, ann. 1801, p. 403. [Expériences sur la phosphorescence du Lampyris noctiluca, L.].

Humboldt, Friedrich-Heimrich-Alexander von, et Bonpland, Aimé. - Voyage au Nouveau-Continent, t. III, 1814, p. 482. [Observat. sur les Pyrophores].

Humboldt, Friedrich-Heinrich-Alexander vox. - Relation historique, t. I, 1814, p. 79 et 533. [Observat. sur les Pyrophores].

Humboldt, Friedrich-Heinrich-Alexander von. - Tableaux. de la Nature, Paris, Gide fils et Baudry, 2 vol., 1850 et 1851, t. II (1851), p. 69. [Observat. sur les Pyrophores]. 
ILLIGER, Johann-Carl-Wilhelm. - Magazin für Insectenkunde. Brunswick, Vieweg., 6 vol., 1802-1807. [Lampyrides, t. IV (1805), p. 195].

ILLIGER, Johann-Carl-Wilheln.-Monographie der Elateren mit leuchtenden Flecken auf dem IIalsschilde, in Magaz. der Gesellsch. der Naturforsch. Freunde zu Berlin, ann. 180\%, t. I, p. 141. [Observat. sur les Pyrophores].

[Analyse in Isis, Encyklop. Zeitschr., Leipzig, ann. 1818, t. IX, p. 1453].

ІмноғF, Ludwig.- Versuch einer Einführung in das s'tudium der Coleopteren, Bàle, Bahnmaier, 2 vol. ar. pl., 1856. [Renseign. peu nombreux sur des Insectes phosphorescents].

\section{J}

Jenner, J.-H.A. - Reappearance of Phosphaenus hemipterus, Gieofir. at Lewes (Angleterre), in The Entomologist, Londres, ann. 1883, p. 216; et in The Entomol. monthl. Magaz., Londres, ann. 1883, t. XX, p. 40. [Phosphorescence et mœurs de ce Lampyride].

Jennivgs, James. — Voir Dale.

Joseph, Gustav. - Beobachtungen über das Leuchten der Johannisliafer, in Breslauer Zeitschr. für Entomol., ann. 1851, t. VIII, 1. 1. [Lampyris nostiluca, 1.]. 
Jousset de Bellesne. - Observation sur la phosphorescence des OEufs tu Lampyre commun, in Compt. rend. hebd. des séanc. de l'Acad. des ścienc., Paris, ann. 1871, t. LXXIII, (séance du 4 septembre 18i1), p. 629. [Lampyris noctiluca, L.].

Jousset de Bellesie. - Recherches expérimentales sur la phosphorescence du Lampyre, in RoBis, Charles, et Pouchet, Georges. - Journ. de l'Anat. et de la Physiol., Paris, ann. I880, t. XVI, p. 121 ; et in Compt. rend. hebd. des séanc. de l'Acad. des scienc., Paris, ann. I880, t. XC, (séance du 16 février 1880), p. 318. [Lampyris noctiluca, L.].

[Extrait in Ann. and Magaz. of Nat. Hist., Londres, ann. 1850, p. 345; in The Entomol. monthl. Magaz., Londres, ann. 1880, t. XVI, p. 214; et in Kosmos, Zeitschr. für einheitl. Weltanschauung auf Grund der Entwicklungslehre, etc., Leipzig, ann. 1880, t. VII, p. 476].

[Analyse in Carus, J.-V.-Zoologisch. Jahresbericht der zoologisch. Station zu Neapel, Arthropoda, Leipzig, ann. 1880, p. 114 ; et in Bertiau, P. - Bericht über Entomol., Berlin, ann. 1880, p. 195].

\section{K}

Kaffer, - Voir Spinola.

Kaiser, Wilhelm. - Ueber das Leuchten von Lampyris splendichla, L., in Anzeig. der kais. Akad. der Wissenschaft.mathemat.-naturwissenschaftl. Classe, Vienne, 3 juillet 1881, p. 133; et in Annal. and Magaz. of Nat. Ilist. [On the Luminosity of the Glow-Worm (Lam- 
pyris splendidula, L.) ], Londres, ann. 1881, p. 372. [Lamprohiza splendidula, L.].

Kawall, H. - Miscellanea entomologica. - Bemerkungen über die Larve von Phosphaenus hemipterus, Geoffr., in Stettiner entomol. Zeit., ann. 1867, t. XXVIII, p. 124. Kerville, Henti Gadeau de. - Voir Gadeau.

KıvG, Helen-Selina. - Life-History of Pleotomus pallens, Le Conte, in Psyche, Journ. of Entomol., published by the Cambridge entomol. Club, Cambridge (EtatsUnis), t. III, 1880, p. 51. [Métamorphoses, biologie et phosphorescence de ce Lampyride].

King, V.-(). - Phosphorescent Insects; their metamorphoses, in The americ. Naturalist, Philadelphie, ann. 1878, t. XII, n 6 , p. 354 .

King, V.-0. - The Fire-Flies and their phosphorescent phenomena, in The americ. Naturalist, Philadelphie, ann. 1878, t. XII, n ${ }^{\circ} 10$, p. 662 .

Lirbi, William, et Spexce, William. - An Introduction to Entomology, or Elements of the natural History of Insects. Londres, Longman, Green, Longman, and Roberts, $7^{\mathrm{m} s}$ édit., 1860, p. 503. (Letter XXV - On luminous Insects). [Nombreux renseign. sur les Insectes phosphorescents].

Kircher, Athanasius. - ? Beobachtungen üher das Johanniskafer, 1610. [Lampyris noctiluca, L.].

Koelliker, Albert. - Ueber die Leuchtorgane von Lampyris, in Verhandl. der physik.-mediz. Gesellsch. in Würzburg, ann. 185\%, t. VIII, $2^{\mathrm{m} s}$ cah., p. $21 \%$. 
[Extrait in Bericht. Verhandl. der Akad. der Wissenschaft. zu Berlin, ann. 1857, p. 392; in L'Institut, Paris, ann. 1857, t. XXV, $\mathrm{n}^{\circ} 1251$, p. 436 ; et in The quarterly Journ. of microscopic. Science, Londres, ann. 1858, t. VI, p. 166].

KoElliker, Albert.-Ueber die Leuchtorgane der amerikanischer Pyrophorus-Arten, in Verhandl. der physik.mediz. Gesellsch. in Würzburg, ann. 1859, t. IX, sitzungsberichte, p. 28.

\section{$\mathrm{L}$}

LaboulbÈne, Alexandre. - Note sur la phosphorescence des Larves et des Nymphes du genre Lampyris, in Annal. de la Soc. entomol. de France, Paris, ann. 1863, p. 470.

LaboulbÈne, Alexandre. - Note sur le Ver luisant (Lampyris noctiluca, L.), in Annal. de la Soc. entomol. de France, Paris, ann. 1882, p. 316. [Cet auteur dit que le Lampyris noctiluca, L. est lumineux à l'état d'œuf, de larve, de nymphe et d'insecte parfait (ơ et $)$ ].

Laboulbène, Alexandre. - Voir Tromelin.

Laboulbène, Alexandre, et Robin, Charles.-Sur les Organes phosphorescents thoraciques et abdominal du Cocuyo de Cuba (Pyrophorus noctilucus, L.), in Compt. rend. hebd. des séanc. de l'Acad. des Scienc., Paris, ann. 1873 , t. LXXVII, (séance du 25 aoùt 1873), p. 511.

Laboulbène, Alexandre, et Robin, Charles. - Observations sur les Organes lumineux du Pyrophorus noctilucus, 
L., in Innal. de la Soc. entomol. de France, Paris, ann. 1873, p. 529.

Lacaze-Duthiers, Félix-Joseph-Henri de. - Recherches sur l'armure génitale femelle des Insectes, in Ann. des Scienc. natur., Zoologie, Paris, ann. 1819, t. XII, p. 353 , et $4 \mathrm{pl}$; ann. 1850 , t. XIV, p. 17, et 3 pl. (Hyménoptères); ann. 1852, t. XVII, p. 206, et 3 pl. (Orthoptères); ann. I852, t. XVIII, p. 337 , et $3 \mathrm{pl}$. (Hémiptères); ann. 1853, t. XIX, p. 25 et 203 , et $4 \mathrm{pl}$. (Neuroptères, Thysanoures, Coléoptères, Diptères, Lépidoptères, Aphaniptères, Généralités). [Armure génitale d'Insectes phosphorescents].

Lacordaire, Jean-Théodore.- Mémoire sur les habitudes des Coléoptères de l'Amérique méridionale, in Annal. des Scienc. natur., Paris, ann. 1830, t. XX, p. 185 et 210, et t. XXI, p. 1 19. [Observat. sur les Pyrophores et les Lampyrides].

Lacordaire, Jean-Thćodore. - Notice sur l'Entomologie de la Guyane fiancaise, in Annal. de la Soc. entomol. de France, Paris, ann. 1832, p. 359. [Pyrophores].

Lacordaire, Jean-Théodore.-Essai sur les C'oléoptères de la Guyane française, in Nouv. Annal. du Muséum d'Hist. natur., Paris, ann. 1833, t. II, p. 35, 66 et 6\%. |Observat. très-sommaires sur les Pyrophores et les Lampyrides].

Lacordaire, Jean-Théodore. - Observations sur la faculté phosphorescente des Fulgores, in Annal. de la Soc. entomol. de France, Paris, ann. 1834, bull. des séanc., p. LXIII. 
LaCordaire, Jean-Théodore. - Introduction à l'Entomologie. Paris, Roret, 2 vol., 1834 et 1838, t. II (1838), p. 140. [Généralités sur la phosphorescence des Insectes].

Lamarck, Jean-Baptiste-Pierre-Antoine dE Monet de. - Sur deux nouveaux genres d'Insectes de la Nouvelle-IIollande (Chiroscelis bifenestrata, Lam. et Panops Bäudini, Lam.), in Annal. du Muséum d'Hist. natur., Paris, ann. 1804, t. II, p. 260, avec fig. [Chiroscelis bifenestrata, Lam., Coléoptère de la famille des Ténébrionides. - Panops Baudini, Lam., Diptère de la tribu des Asiliens].

LAPORTE, F.-L. DE (comte DE CASTELNAU).-Histoire naturelle des Insectes Coléoptères, avec une Introduction renfermant l'anatomie et la physiologie des animaux articulés, par Brullé, Auguste. Paris, Duménil, t. I, 1840, Introd., p. xumi. [Généralités sur la phosphorescence des Insectes].

Lartigue, Henri. - Note sur la phosphorescence de l'Aspidosomus hesperus, Fabr., in Annal. de la Soc. entomol. de France, Paris, ann. 1874, bullet. des séanc., p. CLXI. [Coléoptère de la tribu des Lampyrides].

Latreille, Pierre-André.-Voyage de de Humboldt. Recueil d'observations de zoologie et d'anatomie comparée, faites dans un voyage aux Tropiques, dans les années 1799-1804. Paris, Schoell, 2 vol., 1811-1832. - Insectes, t. I (1811), p. 127, et pl. NV-XXV; et t. II (1832), p. 9. [Observat. sur des Insectes phosphorescents]. 
Latreille, Pierre-André. - Description de la Larve du Lampyris splendidula, L., in Cuvier, Georges. - Le Règne animal. Paris, Deterville, $1^{\text {re }}$ édit., 1817, t. III. [Lamprohiza splendidula, L.].

Latreille, Pierre-André. - Sur la phosphorescence de la tache ocellée qui existe sur chacune des élytres d'un Bupreste de l'Inde (Buprestis ocellata, Fabr.). in Cuvier, Georges. - Le Règne animal, Paris, Deterville, $2^{\text {me }}$ édit., 1829, t. IV, p. 447. [Chrysochroa ocellata, Fabr., Coléoptère de la famille des Buprestides].

Le Conte, John-L. - On lightning Bugs (Lampyridae), in Proceed. of the americ. Associat. for the Advancem. of Science, ann. 1880, p. 650; et in The canad. Entomol., IIontréal, ann. 1880, t. XII, p. 174.

Lefebvre, Alexandre. - Voir Ginmerthal.

LeFebvre, Alexandre. - Voir Hancock.

Lewis, George. - Voir Eaton.

Leydig, Franz. - Lehrbuch der Histologie des Menschen und der Thiere. Francfort, Meidinger, 1857, p. 343, fig. 183. [Structure des organes phosphorescents des Lampyrides].

Lindemann, Carl. - Anatomische Untersuchungen über die Struktur des Leuchtorganes von Lampyris splendidula, L., in Bull. de la Soc. impér. dès Natural. de Moscou, ann. 1863, t. XXXVI, $2^{\mathrm{me}}$ part., p. 437, et pl. VII. [Lamprohisa splendidula, L.].

[Analyse in Gerstaecker, C.-E.-A. - Bericht über Entomol., Berlin, ann. 1863-1864, p. 59]. 
Linden. - Voir Wesmael.

Linsé, Carl vos. - Lyclite-Masken fran China (Fulgora candelaria, L.), in Svenska Vetensk. Acad. Handl., Stockholm, ann. 1746, t. VII, p. 60; in Deutsche Uebers., ann. 1752, t. VIII, p. 61, av. fig.; et in Latein. Uebers., in Analecta transalpina, t. I, p. 475. [Hotinus candelarius, L.]. [Voir DEgEer].

[Cité in Fuessly, J.-C.-Neues Magaz. für die Liebh. der Entomol., Zurich et Winterthur, ann. 1785, t. II, p. 23].

Linné, Carl von. - Miracula Insectorum. Resp. Gabr. Eman. Avelin. 11 novembre 1752. Upsal, Hoyer. [? Observat. sur quelques Insectes phosphorescents].

Loche, François Mouxy DE. - Observations diverses sur les Insectes : III Lampyris italicus, L., etc. in Mém. de l'Acad. roy. des Scienc., Turin, ann. 1801, t. XI, p. 127. [Luciola italica, L.].

Loriquet, Ch. - Sur un Insecte phosphorescent. [Sans lieu ni date].

LuCE. - Description d'un Insecle phosphorique qu'on rencontre dans une partie du district de Grasse, département du Var. (Scarabaeus phosphoricus), in Nouv. Journ. de Physique, Paris, an II (1794), t. I (44), p. 300. [Luciola italica, L.? ou L. lusitanica, Charp.?].

\section{M}

Macaire, Isaac-François. -Sur la phosphorescence des Lampyres, in Biblioth. univers. des Scienc., Belles-Lettres et Arts, Genève, ann. 1821; in Journ. de Physique, 
Paris, ann. 1821, t. XCIII, p. 46 ; in Annal. de Chimie et de Physique, Paris, ann. 1821, t. XVII, p. 251 ; in Froriep, L.-F. vos. - Notizen aus dem Gebiete der Natur- und Heilkunde, Weimar, ann. 1821, t. I, p. 33; in Giorn. di Fisica, Chimica e Storia naturale, etc., Pavie, ann. 1821, p. 321 ; et in Gilbert, L.-IV. - Annal. der Physik, ann. 1822, t. LXX, p. 265.

Macartney, James. - Observations upon luminous Animals, in Philosoph. Transact. of the roy. Soc. of London, ann. 1810 , t. C, p. 258 et 277 , et pl. XV, fig. 13-16 (Lampyris), fig. 17-19 (Pyrophorus); et in GiLbert, L.-IV. - Annal. der Physik, ann. 1819, t. LXI, p. 1 et 113 , av. 2 pl. (Notes de Tilesius).

[Extrait in The philosoph. Magaz., Londres, ann. 1811, t. XXXVII, p. 24 et 93, av. 2 pl.; et in Abstracts of the papers printed in the Philosoph. Transact. from 1800 1830, Londres, 1832, t. I, p. 379. [Observat. sur des Insectes phosphorescents].

Mac Lachlan, Robert. - Glow-Worms v. Snails, in Nature, Londres et New-York, 1879, t. XX, $\left(\mathrm{n}^{\circ} \mathrm{du}\right.$ 3 juillet 1879), p. 219. [Lampyris noctiluca, L.].

MaC Lachlan, Robert. - Voir Clark.

Mac Laurin, Wm. - The Glow-Worm in Scotland, in Nature, Londres et New-York, 1876, t. XIII, $\left(\mathrm{n}^{\circ} \mathrm{du}\right.$ 13 janvier 1876), p. 208, ]Lampyris noctiluca, L.].

Mallle, Arsène. - Note sur les habitudes naturelles des Larves de Lampyres, in Annal. des Scienc. natur., Paris, ann. 1826, t. VII, p. 353. 
[Extrait in Nouv. Bull. de la Soc. philomat., Paris, ann. 1826, p. 26; in Férussac, $\Lambda$. DE.-Bull. univers. des Scienc. natur. et de Géologie, Paris, ann. 1826, t. VIII, p. 296; in Froriep, L.-F. vos. - Notizen aus dem Gehiete der Natur- und Heilkunde, Weimar, ann. 1826, t. XIII, p. 321 ; in SaIGeY, J.-F. et Raspall, F.-V. Annal. des Scienc. d'Observat., Paris, ann. 1829, t. II, p. 299, (par M.M. de Rouen); et in Isis, Encyklop. Zeitschr., Leipzig, ann. 1834, t. VII, p. 850].

Main._Sur une Tipula oleracea, L. lumineuse? in Magaz. of Nat. Hist., Londres, ann. 1837, p. 519. [Diptère de la famille des Tipulides].

MaIN, James. - Voir DALE.

Mairan, Jean-Jacques, Dortous DE. - Voir Dortous.

Martin. - Voir Austin.

Martire d’Axghiera, Pietro. - Voir Anghiera.

Martius, Carl-Friedrich-Phil. vox. - Voir Spix.

Matteucci, Carlo. - On the phosphorescence of the Lampyris italica, L., (extract from a letter to Dumas), in Annal. and Magaz. of Nat. Hist., Londres, ann. 1843, t. XII, p. 373; in Froriep, L.-F. et R. vox. - Neue Notizen aus dem Giebiete der Natur- und Heilkunde, Weimar, ann. 1S13, t. XXVII, p. 168; et in Compt. rend. hebd. des séanc. de l'Acad. des Scienc., Paris, ann. 1843, t. XVII, (séance du 14 août 1843), p. 309. [Luciola italica, L.].

[Analyse in Erichsos, W.-F. - Bericht über Entomol., Berlin, ann. 1813, p. $23(\because 71)]$. 
Matteucci, Carlo. - Leçons sur les phénomènes physiques des corps vivants. Paris, V. Masson, 1847, p. 151. [Expériences et Observat. nombreuses sur la phosphorescence du Luciola italica, L.].

[Analyse in Schleiden, M.-J. et Froriep, R. von. Notizen aus dem Gebiete der Natur- und Heilkunde, Weimar, ann. 1847, t. I, p. 135].

Maurer, Felix. - Vom Lichte der Johanniswürmlein, in Observat. Curios. Phys., p. 490. [Lampyrisnoctiluca, L.].

Melchion, Johann-Albert. - De Noctilucis. Dissert. philosoph. inaugur., Franequerae, 1742. [Observat. sur des Insectes phosphorescents].

Meldola. - Spectrum of the light of the Glow-Worm, in Nature, Londres et New-York, 1882, t. XXVI, $n^{\circ} 667$. [Lampyris noctiluca, L.].

Merian, Maria-Sibylla. - Dissertation sur la génération et les métamorphoses des Insectes de Surinam. 1726, p. 49, av. fig. [Phosphorescence du Fulgora laternaria, L., observée par l'auteur].

Milne-Edwards, Henri. - Lecons sur la physiologie et l'anatomie comparée de l'Homme et des animaux. Paris, Masson, 14 vol., 1857-1881, t. VIII (1863), p. 95. [Renseig. nombreux sur la phosphorescence des Insectes].

Mocquerys, Emile._Observations recueillies au Brésil sur quelques Pyrophores et Lampyrides, in Annal. de la Soc. entomol. de France, Paris, ann. 1844, bull. des séanc., p. LxiII. 
Monet de Lamarck, Jean-Baptiste-Pierre-Antoine de. - Voir LAMARCK.

Montbeillard, Philibert Guéneau De. - Voir Guéneau.

Montrouzier. - Essai sur la faune entomologique de la Nouvelle-Calédonie, in Annal. de la Soc. entomol. de France, Paris, ann. 1860, p. 229 et 867 , av. 1 pl. [Observat. sur des Insectes phosphorescents].

Morren, Charles-François-Antoine. - Sulla fosforescenza delle Lampiridi noctiluca, L. e splendidula L., in Atti terza riunione Scienz. ital., Florence, ann. 1841, t. IV, p. 366 ; et in Isis, Encyklop. Zeitschr., Leipzig, ann. 1843, t. VI, p. 412. [Lampyris noctiluca, L. et Lamprohiza splendidula, L.].

MORRIS. - Voir BÉtIIUNe.

Moufet, Thomas. - Insectorum sive Minimorum Animalium Theatrum, etc. Londres, T. Cotes, 1634, lib. I, cap. 15. [? Pyrophores].

Moufflet, Alfred. - Note sur la phosphorescence du Fulgora laternaria, L., in Annal. de la Soc. entomol. de France, Paris, ann. 1865, bull. des séanc., p. Lxir. [Moufflet dit avoir observé lui-même la phosphorescence de cet Hémiptère].

Mouxy de Loche, François. - Voir Loche.

Mueller, Philipp-Wilbrand-Jacob. - Beitraege zur Naturgeschichte des halbdeckigen Leuchtkajers Lampyris hemiptera, in ILLIGER, J.-C.-IV. - Nagaz. für Insectenkunde, Brunswick, t. IV (I805), p. 175. [Description 
de la larve du Phosphaenus hemipterus, Geoff., Coléoptère de la tribu des Lampyrides, p. 182].

Mulsant, Etienne. - Observations sur les Lampyrides, (avec Eugène Revelière), in Opuscul. entomol., Lyon, 1860, t. XI, p. 113; et in Annal de la Soc. linn. de Lyon, ann. 1861, p. 129. [Lampyris bicarinata, Muls.].

Mulsant, Etienne. - Histoire naturelle des Coléoptères de France.-Mollipennes, in Ann. de la Soc. linn. de Lyon, ann. 1862, p. 57. Tir. à part, Paris, 1863. [Observa1. sur les Lampyrides; description et figuration des larves des Lampyris Lareyniei, Jacq. du Val, L. noctiluca, L., Lamprohiza Farinesi, Villa, et Phosphaenus hemipterus, Geoffr.].

Mulsant, Etienne. - Note sur les habitudes de la Luciola lusitanica, Charp., in Annal. de la Soc. linn. de Lyon, ann. 1862, p. 593 .

Muralto, Johann von. - De Cicindela (Lampyris femina aptera), in Miscellan. curiosa, sive Ephemer. medicophysic. Acad. Caes. Leopold.-Carolina Natur. Curios., Nuremberg, ann. 1682, décemb. II, ann. I, observat. 67, p. 167.

Murray, Andrew. - On an undescribed lightgiving Coleopterous Larva (provisionally named Astraptor illuminator), in Journ. of the Proceed. of the linn. Soc. of London, Zoology, ann. 1868, t. VIII, p. 74, et pl. I, fig. 1-7; et in The Entomologist, Londres, ann. 1869, t. IV, p. 281. [Larve de Lampyride?]. 
Murray, John. - Experimental Researches on the light and luminous matter of the Glow-Worm, the Luminosity of the sea, the Phenomena of the Chameleon, etc., Glasgow, M'Phun, 1826. [Lampyris noctiluca, L.].

Murray, John. - On luminous Insects, in Experimental Researches in natural History, Londres, 1826. (Extr. de l'ouvrage précédent). [Lampyris noctiluca, L.].

[Extrait in Froriep, L.-F. von. - Notizen aus dem Gebiete der Natur- und Heilkunde, Weimar, ann. 1827, t. XVI, p. 122; in Heusinger, C.-F. - Zeitschr. für organ. Phys., Eisenach, ann. 1828, t. II, p. 94; et in Magaz. of Nat. Hist., Londres, ann. 1828, p. 155 et 298].

\section{$\mathrm{N}$}

Napp, Richard. - Die argentinische Republik. BuenosAires, 1876. [Coup d'œil sur la faune des Arthropodes de la République argentine, par Weyenbergh, $\mathrm{H}$. (p. 172-186). - Observat. sur des Insectes phosphorescents].

[Analyse in Bertkau, P. - Bericht über Entomol., Berlin, ann. 1877-1878, p. 2 (220)].

Naddis, Charles-Victor.-Observations sur le Lamprohiza Mulsanti, Kiesw., \&, et sur sa phosphorescence, in Annal. de la Soc. entomol. de France, Paris, ann. 1869, bull. des séanc., p. xxxv. [Coléoptère de la tribu des Lampyrides ].

Newall, Robert-Stirling. - Snails v. Glow-Worms, in Nature, Londres et New-York, 1879, t. XX $\left(\mathrm{n}^{\text {os }}\right.$ des 
26 juin et 10 juillet 1879), p. 197 et 243. [Lampyris noctiluca, L.].

Newdan. - Sur la non-phosphorescence du Fulgora candelaria, L., in Transact. of the entomol. Soc. of London, ann. 1864, proceed., p. xiv. [Hotimus candelarius, L.].

Newman, E. - Voir Walker.

Neuwied, Maximilian von IVIed-. - Reise nach Brasilien, t. II, 1820, p. 111. [Observat. sur le Fulgora laternaria, L.]. [Cet auteur nie la phosphorescence de cet Hémiptère].

Newport, George. - On the natural History of the GlowWorm (Lampyris noctiluca, L.); prepared from the author's manuscript by George-Viner ElLIs, in Journ. of the Proceed. of the linn. Soc. of London, Zoology, ann. 1856, p. 40.

[Analyse in Gerstaecker, C.-E.-A. - Bericht über Entomol., Berlin, ann. 1857, p. 110].

Nieremberg, Johann-Eusebius.-Historia Naturae, maxime peregrinae, libris sedecim distincta. Antwerpiae, Plant, 1635, lib. XIII, cap. 3. TObservat. sur des Insectes phosphorescents].

Nollet, Jean-Antoine.-Suite des Expériences et des $O b$ servations faites en différents endroits de l'Italie, in Mém. de l'Acad. roy. des Scienc., ann. 1750. Paris, 1754. Ném. de l'Acad., p. 54; Hist. de l'Acad., p. 7. [Observat. sur le Luciola italica, L.]. 
Norwood. - Observations in Jamaïca, in Philosoph. Transact. of the roy. Soc. of London, ann. 1668, $n^{\circ} 41$, p. 824. (Memoir. of the roy. Soc. of London, t.I, p. 152). [Observat. sur des Insectes phosphorescents].

\section{$\mathrm{O}$}

Olivier, Antoine-Guillaume. - Observations sur le genre Fulgore, in Choix de mémoir. sur divers objets d'Hist. natur., ou Journ. d'Hist. natur., Paris, ann. 1792, t. II, p. 31; et in MEYER. - Zoologisches Archiv, ann. 1796, t. II, p. 30. [Olivier est le premier auteur qui ait mis en doute la propriété phosphorescente de cet Hémiptère].

OLivier, Ernest. - Lampyrides nouveaux ou peu connus, in Revue d'Entomol., publiée par la Soc. franç. d'Entomol., Caen, ann. 1883, p. 73 et 326. [Descript. de la larve de la Luciola australis, Fabr., p. 331].

Olivier, Ernest. - Description de la Larve du Lampyroüdea syriaca, Cost., in Annal. de la Soc. entomol. de France, Paris, ann. 1886, bull. des séanc., p. xxxvill. [Coléoptère de la tribu des Lampyrides].

Olivier, Ernest. - Voir Tromelin.

Osten-Sacken, Charles-Robert von. - Entomologische Notizen, in Stettiner entomol. Zeit., ann. 1861, t. XXII, p. 51. [Caractères de la phosphorescence et mœurs de quelques Lampyrides nord-américains : Photinus pyralis, L.; Phol. scintillans, say; et Photuris pensylvanica, Degeer]. 
Ostris-Sackex, Charles-Robert von. - Note sur une Larve lumineuse, in Proceed. of the entomol. Soc. of Philadelphia, ann. 1862, t. I, p. 125, et pl. I, fig. 8 . [Dans le travail suivant, lauteur considère cette larve comme étant très-probablement celle d’un Melanactes, Coléoptère de la famille des Elatérides].

Osten-Sacken, Charles-Robert von. - Note sur une Larve lumineuse, in Proceed. of the entomol. Soc. of Philadelphia, ann.1865, t. IV, p. S. [Cette larve, suivant l'auteur, est très-probablement celle d'un Melanactes, Coléoptère de la famille des Elatérides].

Osten-Sacken, Charles-Robert von. - Luminous Diptera, in The Entomol. monthl. Magaz., Londres, ann. I878, t. XV, p. 43. [Larves lumineuses de Chironomus, Diptères de la famille des Chironomides].

Osten-Sacken, Charles-Robert von. - A luminous InsectLarva in New-Zealand, in The Entomol. monthl. Magaz., Londres, ann. 18s6, t. XXIII, p. 133. [Larves d'un Diptère appartenant très-probablemnent à la famille des Mycétophilides]. [Voir Hudson].

Osten-Sacken, Charles-Robert von. - Voir Bétuune.

Oviedo y Valdes, Gonçalo-Fernandez de. - Sumario de la natural y general Istoria de las Indias. Tolède, 1526. [Observat. sur des Insectes phosphorescents].

Oviedo y Valdes, Gonçalo-Fernandez de. - Historia general y natural de las Indias Islas y tierra firme del mar Oceano, publicadu por la real Academia de la 
Historiu, part. I et II. Madrid, 1851 et 1852. (Livr. XIV et XV). [Observat. sur des Insectes phosphorescents].

Owsiannikoff, Philipp. - Ueber das Leuchten der Laiven von Lampyris noctiluca, L., in Bull. de l'Acad. impér. des Scienc. de Saint-Pétersbourg, ann. 1864, t. VII, p. 55.

[Analyse in Gerstaecker, C.-E.-A. - Bericht über Entomol., Berlin, ann. IS63-1864, p. 236].

Owsiannikoff, Philipp. - Ein Beitrag zur Kenntniss der Leuchtorgane von Lampyris noctiluca, L., in Bull. de l'Acad. impér. des Scienc. de Saint-Pétersbourg, ann. 1S68, t. XII, $\mathrm{n}^{\circ} 17$, p. 12, av. 1 pl.

\section{$\mathrm{P}$}

Palisot de Beauvors, Ambroise-Marie-François-Joseph. Insectes recueillis en Afrique et en Amèrique, dans les royaumes d'Oware, à Saint-Domingue et dans les Etats-Unis, pendant les années 1781-1797. Paris, Levrault, 1805-1821, av. de nombr. pl. color. [Observat. sur des Insectes phosphorescents].

Pallas, Peter-Simon. - Kleine Notizen in den neuen nordischen Beitraegen. (Ueber das Leuchten von Lampyris und Culex). 1782, t. IV, p. 396). [Culex, Diptères de la famille des Culicides].

Parfitt, Edward. - On the phosphorescence of the GlowWorm, in The Entomol. monthl. Magaz., Londres, ann. 1880, t. XVII, p. 94. [Lampyris noctiluca, L.]

Parzudaki. - Voir Reiche. 
Pasteur, Louis et Gernez. - Sur la lumière phosphorescente des Cucuyos, in Compt. rend. hebd. des séanc. de l'Acad. des Scienc., Paris, ann. 1864, t. LIX, (séance du 19 septembre 1864), p. 509. [Pyrophorus noctilucus, L.]. [Remarques sur cette communication par Emile Blanchard, p. 510].

Penny, R. Greenwood. - Voir Greenwood.

Peragallo, Al.- Note pour servir à l'Histoire des Lucioles, in Annal. de la Soc. entomol. de France, Paris, ann. 1862, p. 620. [Luciola lusitanica, Charp.]. [Note de Louis Reiche, p. 624].

Peragallo, Al. - Seconde Note pour servir à l'Histoire des Lucioles, in Annal. de la Soc. entomol. de France, Paris, ann. 1863, p. 661. [Luciola lusitanica, Charp.]. [Cet auteur parle (p. 663) d'un Insecte en tout semblable à un Staphylinus olens, Müller de forte taille, qui laissait derrière lui une trace lumineuse. Il admet: " ou qu'il existe à Menton un Staphylin phosphorescent vivant de Lucioles ", ce que je ne puis croire, ou que les individus rencontrés par lui et son compagnon de chasse, "se trouvaient enduits de la pâte phosphorescente et très-persistante qui emplit les deux derniers anneaux de l'abdomen des Lucioles qu'ils venaient de manger »; cette dernière explication me parait seule admissible].

[Analyse in Gerstaecker, C.-E.-A. - Bericht über Entomol., Berlin, ann. 1863-1864, p. 237].

Peragallo, Al. - Voir Tromelin.

Percheron, Achille-Rémy. - Note sur trois Insectes lumineux, in Silbermans, G. - Revue citomol., ílras- 


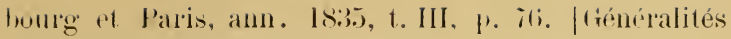
sur des Insectes phosphorescents|.

Perkins, G.-A. - The Cucu!jo, or mest-indian FireBeelle, in The anteric. Naturalist, Salem, ann. 1870, t. III, p. 12.2. [Pyrophorus nortilucus, L.].

Pertr, Maximilian. - Delectus animulium articulatorum, qune in ilinere per Brasilirm annis 1S17-1820 jussu et anspinies Maximiliani Josephi Bavarine regis augustissimi peracto, collegerunt $I^{\mathrm{r}} J .-B$. de spix et $D^{r}$ C.-F.-Pl. de Martius; digessit, descripsit et pingenda curavit $D^{r}$ M. Perty. Munich, auctor, 2 vol., 1830-1834. [Observat. sur des Insectes phosphorescents].

Peters, Wilhelm-Carl-Hartwig. - Ueber das Leuchten dep Lamp!gris ilalica, L., in Nubler, J. - Mrchiv für Anat., Physiol. und wissenschaftl. Medicin, Berlin, ann . 1811, p. 229; in Annal. des Scienc. natur., Zoolog., Paris, ann. 18 12, p. 251; in Revue zoolog., par la Soc. curiérienne, Paris, ann. 1812, t. V, p. 22:3 in L'Institut, Paris, ann. 1842, t. X. $n^{\circ} 432$, p. 127; et in Edinburgh new philosoph. Journ., ann. I843, t. XXXIV, p. 30. [ Luciola italica, L.].

[Analyse in Erichsox, W'.-F. - Bericht über Entomol., Berlin, ann. 1811, p. $28(216)]$.

PrLuger, E.-F.-IV. - Die Phosphoresceñ der lebendigen Organismen und ilıre Bedeutung für die Principien der Respiration, in Pfluger, E.-F.-IV.-Archiv für die gesammte Physiologie des Menschen und der Thiere, Bonn, ann. 1875, t. X, p. 275. [Observat. sur la phosphorescence des Insectes]. 
Pfluger, E.-F.-II.-Ceber die Phosphorescensierliesender Organismen, in Pfluger, E.-F.-IV. - Archiv für die gesammte Physiologie des Menschen und der Thiere, Bonn, ann. 1875, t. XI, p. 222. [Observat. sur la phosphorescence des Insectes].

Phipson, T.-L. - De la phosphorescence en général et cles Insectes phosphoriques en priticulier, in Journ. publié par la soc. des scienc. médical. et natur. de Bruxelles, Bruxelles, J.-B. Tircher, 1858, (tirage à part).

Phipson, T.-L. - Phosphorescence, or the Emission of Light b! Minerals, Plants and Animals. Londres, Reeve et C', 1s62. [Observat. sur des Insectes phosphorescents].

Phipsox, T.-L. - Sur quelques Insectes phosphorescents, in Cosmos, Paris, 26 juillet 1865 ; et in Guéris IIÉxEville, F. - Rev. et Magas. de Zoolog. pure et appliquée, Paris, ann. 1865, p. 251. [Observat. sur le Lamprohia splendidula, L. et sur les Pyrophores].

Pickinan Ilans, B. - Notes on luminous Larvae of Elateridae (Asaphes memnonius, Herbst), in Psyche, Journ. of Entomol., published by the Cambridge entomol. Club, Cambridge (Etats-Unis), 1875, t. I, p. 89. [Larves d'Elatérides?].

Plinius Secundus, Caïus. - Histoire naturelle. Traduction française. Paris, Desaint, 12 vol., 1771-1782, t. IV (1772), liv. XI, chap. 28, p. 309; t. VI (1773), liv. XVIII, chap. 27, p. 491. [Observat. sur des Lampyrides]. 
Pocjane, Gustave-Arthur. - Nole sull le bioleggie dlu Lampygris noctiluca, L., \&, in Annal, de la soc. entomol. de France, Paris, ann. 1883, bull. les séanc., p. Lxixvir.

Prter, W.-B. - Tropical Notes, in The Entomol. monthl. Iagaz., Londres, ann. 18s0, t. NIII, p. 211. |Observat. sur des Insectes phosphorescents. - Lauteur nie la propriété phosphorescente des Fulgorides, p. 211].

\section{$\mathrm{R}$}

Radziszersis. - Ueber die Phosphorescen: der organischen und organisirten Korper, in Annal. ller Chemie, IS80, t. CCIII, p. 305. [Observat. sur la phosphorescence des Insectes].

Ramsden. - Merurs du Pyrophome causlicus, Germ., in Transact. of the entonol. Soc. of London, ann. 1880 , proceed., p. xrxı.

RaY, John. - Historia Insectorum; opus posthumum. Cui subjungitur appendix de Scarabaeis lritannicis auctore M. Lister. Londres, Clurchill, 1710, p. 81. [Observat. sur le Lamp!yris noctiluca, L.].

RAY, John. - Philosophical letters between $\boldsymbol{M}^{\mathbf{r}}$ Ra!j and several of his correspondents. Londres, 1718 (édit. de Derham). [Observat. sur des Insectes phosphorescents].

Razounowsir, Georg von. - Histoire naturelle du Jorat et de ses encirons, et celle des trois lacs de Neufchitel, Morat et Bieme. Lausanne, Hourer, 2 vol., 1789. t. I, 
p. 159, (note). [Observat. sur la phosphorescence des Lampyres].

Razounowski, Georg von. - Mémoire sur le Ver-luisant, in Némoir. de la Soc. des Scienc. physiq. de Lausanne, ann. 1789, t. II, part. 1, p. 210, avec 1 pl. Lampyris noctiluca, L.|.

Réaumur, René-Antoine Ferchallt de. - Mémoiles pour servir à l'Histoive des Insectes, 6 vol., av. pl., Paris, imprin. roy., 1731-1742. T. V (1710), p. 192, et pl. XX, fig. 6-9. [Observat. sommaires sur le Fulgora laternaria, L.].

Recluz. - Sur les habiludes du Lampyjris noctiluca, L., in Saigey, J.-F. et Raspall, F.-V. - Annal. des Scienc. d'Ubservat., Paris, ann. 1829, t. II. p. 299.

Regnard, Paul. - Voir Dubois.

Reıche, Louis. - Détails sur la crépitation des Brachinus et sur lu luen' phosphorescenle qui l'accompagne, in Annal. de la Soc. entonol. de France, Paris, ann. 1849, bull. des séanc., p. Lx. [Observat. analogues de PArzuDaki et de J.-H. Rovzet sur le inême sujet].

Reiche, Louis.-Note sur quelques Lares de Lampyrides. in Annal. de la Soc. entomol. de France, Paris, ann. 1863, p. 476. [Observat. sur les larves de diverses espèces de Lampyrides, et descript. de la larve du Lamprohiza Delarouseei, Jacq. du Val].

[Analyse in Gerstaecker, C.-E.-A. - Bericht über Entomol., Berlin, ann. 1863-1861, p. 236]. 
Reiche, Louis, - Voir Peragallo.

Reixhardt, Johann-T. - Twende Jayllagelser "f phosphorisk Lysning hos en Fisk og en Insectlarve, in Vilensk. Meddel. fra d. naturhist. Forening i Kjohenhavn, ann. 1853 , p. 60 , - tir. à part; in Transact. of the entomol. Soc. of London, ann. I851, proceed., p. v; et in Zeitschr. für die gesammt. Naturwissenschaft., Berlin, ann. 155.5, t. V, p. 208. [Larve de Coléoptère lumineuse|.

Revelière, Eugène. - Voir Mulsait.

Rer, Claudius. - Description de la Larve de la Lamprohisa Mulsunli, Kiesw., in Annal. de la soe. limı. de Lyon, ann. 1882, p. 113.

Riley, Ch.-V. - Ioir TromeliN.

Ruvints, Quir.-Sept.-Flor.-Dissertutio de noctu lucentibus. Leipzig, 1673. |Observat. sur des Insectes phosphorescents|.

Robert, Eugène. - Observations diver'ses relatives à des Insectes des environs de Puris. (Lampyris, etc.), in Annal. des Scienc. natur., Zoolog., Paris, ann. 1812, p. 378.

[Analyse in Ericusox, W.-F. - Bericht über Entomol., Berlin, ann. 18 12, p. 32 (176)].

Roвевт, Eugène.-Su la phosphorescence du Ver-luisant, in Compt. rend. hebd. des séanc. de l'Acad. des Scienc., Paris, ann. 1813, t. MVII, p. 62\%. [Lamp!yris noctiluere, L.]. 
Robis, Charles. - Voir Laboulbène.

Robiceau-Desvoidy, André-Jean-Baptiste. - Note sur le Thyreophora cynophila, Panz., in Annal. de la Soc. entomol. de France, Paris, ann. 1841, p. 273. [Diptère de la famille des Muscides].

Robineau-Desvoldy, André-Jean-Baptiste. - Note sur le genre Thyreophora et sur les trois especes qui le composent, in Annal. de la Soc. entomol. de France, Paris, ann. 1849, bull. des séanc., p. v. [Expériences tendant a faire nier la phosphorescence du Thygreophora cynophila, Panz., Diptère de la famille des Muscides].

Rochefont, César be. - Histoire nalurelle et morale des Iles Autilles de l'Amérique. Rotterdan, 1658. [01,servat. sur des Insectes phosphorescents].

Rogerson, W. - On the Glow-Hom, in The philosoph. Magaz., Londres, ann. I821, t. LVIII, p. 53; et in Isis, Encyklop. Zeitschr.. Leipzig, ann. 18:31, cah. V, p. 156. [Lampyris noctiluca, L.].

Rolzet, J.-II. - Voir Reiche.

\section{S}

SAxa, John. - Luciola italica, L. at Darlington (Angleterre), in The Entomol. monthl. Magaz., Londres, ann. 1885 , t. XXII, p. 138.

Saunders. - Voir Clark. 
Scaliger, Julius-Carsar. - Exotericarum exercilutionum liter $X V$ de subtilitate ad II. Cardanum. Paris, I557, fol. cxcir, nos 1 et $3 . \mid L a m m ! y)$ is $\mid$.

Schloedte, Joergen-Christian, - Voir smitu.

Scunid, Carl-August. - Versuche über die Insecten. Ein Beitra!y sur Verbreitung des nülslichen und wissenswïrdigen aus der Insectenliunde. Gotha, Ettinger, 1803, t. 1, p. 245. [Lampyris noctilucu, L.].

Schneider, Wilhelm-Gottlieb. - Ueber seltene Coleoptera. (Newport. - Ueber Lampyris), etc., in Arbeit. der schlesig. Gesellsch. für vaterl. Kultur, Breslau, ann. 1859 , p. I.

Schnetzler, J.-B. - Le la produclion de la lumière che: les Lampyles, in Irchiv. des scienc. physiq. et natur., Genève et Paris, ann. 1855, t. M.XX, p. 223.

|Analyse in Genstaecker, C.-E.-A. - Bericht über Entomol., Berlin, ann. I856, p. i9].

Schultze, Max-Sigismund. - Ueber den Bau der Leuchtorgane der Mannchen von Lampyris splendidula, L., in sitzungsberichte der niederrhein. Gesellsch. für Natur- und Heilkunde zu Bonn, amn. 1861, p. 61. [Lamprohisa splendidula, L.].

[Analyse in Gerstaecker, C.-E.-A. - Bericht über Entomol., Berlin, ann. 1863-1861, p. 57].

Schultze, Max-sigismund. - Zur Kemlniss der Leuchtoryane von Lampyris splemdidule, L., in Schultze, M.-S. - Archir für mikroskop. Anatomie, Bonn, ann. 1865, t. I, p. 12.1, et pl. Vet VI. |Lamprohisa splendidula, L.]. 
[Analyse in Gerstaecker, C.-E.-1. - Bericht über Entomol., Berlin, ann. I865-1866, $1^{\text {re }}$ part., p. 16].

SELLs, William. - Observations upon the natural History of tririous species of West-India Insects, in Transact. of the entomol. Soc. of London, ann. IS35, proceed., p. xur. [Observat. sur le Pyrophorus noctilucus, L., etc.].

Serern, H.-A.-Notes on the imlind Glow-Fly. in Nature, Londres et New-lork, I881, t. XXIV ( $n^{\circ}$ du 23 juin 1881), p. I65. [Lampyride?].

[Analyse in MAYer, P. - Zoologisch. Jahresbericht der zoologisch. Station zu Neapel, Arthropuda, Leipzig̈, ann. 1881, p. 128].

sinap. - Note sur la phosphorescence de la Luciola lusitanica, Charp., in The Entomol. monthl. Magaz., Londres, ann. 1880, t. XVII, p. 69.

Snaw, John. - The Glow-Worm, in Nature, Londres et New-York, 18i6, t. XIII (n' (lu 6 janvier 1876), p. 188. [Lamp!yris noctiluca, L.].

Shufeldt, Robert-II. - Notes on various Coleoplera of Neu'-Urlerus, in Proceed. of the United States nation. Museum, Washington, ann. 1881, t. VII, p. 331, ar. fig. [Larres lumineuses de Melanactes? et d'Asaphes?, Coléoptères de la famille des Elatérides, p. 33i, av. fig.].

Sigwart, Georg-Friedrich, et WestFeld, Chr.-FriedrichGothart. - Von dem stheinumme, Contharis noctilucu, L., in Neues hamburg. Magaz., etc., Hambourg, ann. 1768, t. IV, p. 58. [Lampyris noctiluce, L.]. 
SLoave, Hans. - A Voyage to the Islands Madera, Barbades, Nieves, Snint-Christophers and Jamuïca, etc. Londres, auteur, 2 vol. av. nombr. planch., 1707 et 1725 ; t. II (1725), p. 206. [Observat. sur les Pyrophores].

Surn, James. - Sur la phosphorescence du Fulgor candelaria, L., in Transact. of the entomol. soc. of London, ann. 1861, proceed., p. xir. [Hotimes candelarius, L.]. [Cet auteur lécrit la phosphorescence de cet Hémiptère]. [Analyse in Gerstaecker, C.-E.-A. - Bericht über Entomol., Berlin, ann. 1863-1864, p. 61].

Simн, James. - Larve of Pyrophorus (?) firom Lruguay, in Transact. of the entomol. Soc. of London, ann. 1869, proceed., p. xv. [E. CAndèze et Joergen-Christian schiogdte (Proceed. indiqués ci-dessus, p. xvi) considèrent cette larve conme étant celle d'un Elatéride, et pensent l'un et l'autre que l'Astraptor illuminator, Murray n'est pas un Pyrophore].

[Analyse in Brauer, F. - Bericht über entomol., Berlin, ann. 1869, p. 10].

Surth, James. - Sur la pliosphorescence des Fulyores, in Transact. of the entomol. Soc. of London, ann. 1871, proceed., p. vil. [Cet auteur croit que les Fulgores sont phosphorescents|.

Solmalerring, S.-T. rox. - Voir Forster.

Sora, Franz-Lothar-August-IVilhelm.-Disquisitiones physiologicae circa respirationem Insectorum et Vermium. Rudolstadt, Langbein, 1805, 2 part., p. 35. Johservat. sur la phosphorescence des Lampyres]. 
Spallavzay, Lazzaro.-Chimico Essame degli Esperimenti del sign. Gottling a Iena sopra la luce del fosforo di Kumlel, etc. Morlène, 1796, p. I19. [Observat. sur la Luciola itulica, L.].

Spexce, William. - On the Luminosity of Fulgora laternaria, L., in Transact. of the entomol. soc. of London, ann. 1818, proceel., p. xxxvin.

Spexce, William. - Voir linbY.

Spiller. - Voir Cosror.

Sprixola, Naximilian. - Essui sur les Fulyorelles, soustribu des Cicarlaires, ordre des Rhyngotes, in Annal. de la sinc. entomol. dr France, Paris, ann. 1839, p. 135, av. 8 pl. col. - Tir. à part, Gènes, 1839, 2 rol., ar. 8 pl. col. [Observat. sur la phosphorescence des Fulgorides].

[Extrait in Revue zoolog., par la sococ. curiérienne, Paris, ann. 1839, t. II, p. 199|.

Sprout, Iaximilian. - Sur la phosphorescence du Fulgora laternaria, L., in Rerue zoolog.. par la Soc. cuviérienne, Paris, ann. 1s 14, t. VII, p. 210. [Spinola dit qu'un voyageur, nommé Kiffer, prétend aroir vu un exemplaire phosphorescent de cet Hémiptère].

SPIX, Johann-Baptist vox, et MarTús, Carl-Friedrich-Phil. vox. - Travels in Brail, in the years 18Ii-1820. Londres. Longman, 2 vol., 1821. |Observat. sur des Insectes phosphorescents]. 
Stedman, John-Gabriel. - Einige Bemerkungen über guianische Insecten, in IlLIGER, J.-C.-W. - Magaz. für Insectenkunde, Brunswick, 1. IV (1805), p. 226. | Cet auteur arlmet la phosphorescence du fiulgora laternaria, L.].

Steinherl, E. - Note sur des indivirhs reliant le Pyrophorus pellucens, Esch. au P. rlarus, Germ., avec des observations sur l'intensité de la lumière de ce dernier, in Harold, E. vox. - Coleopter. Hefte, t. XIV, p. 132.

Stillin, W -.J. - Glow-Worms, in Nature, Londres et NewYork, 1883, t. XXVIII, p. 243. [Ròle de la phosphorescence chez la femelle du Lamprohisa splendidula, L.].

SToLlwerck, F. - Zoologyisrhe Villheilungen, in Verhandl. des naturhist. Verein. der preuss. Rheinl. und Westlal., Bonn, $-10^{\circ}$ ann., 1883, p. 428. [Observat. sur un Pyrophorus noctilucus, L. importé en Europe dans du bois de teinture américain, p. 431].

Strickland, Hugh-Edwin. - On the Luminosity of GlowWorm's Eggs, in Magaz. of Nat. Hist., Londres, ann. I831, p. 252. [Phosphorescence des œufs du Lamp!jris noctiluca, L.].

Strickland, Hugh-Eiwin. - Voir Dale.

StubBes. - A continuation of the Voygage to Jamaïca, in Philosoph. Transact. of the roy. Soc. of London, ann. $1667, n^{\circ} 36$, p. 699. (Memoir. of the roy. Soc. of London, $2^{\circ}$ édit., 1745, p. 131); et in Journ. des sicavants, 166i. [Observat. sur des Insectes phosphorescents]. 
Stubbes. - Enlargement of the Observations in a Voyage to the Caribes, in Philosoph. Transact. of the roy. Soc. of London, ann. 1668, t. III, n 36, p. 706. [Observat. sur les Pyrophores].

Swamerdam, Johann. - Biblia Naturae. Leyde, Severin, 2 vol., 1737 et 1738, t. I, p. 283. [Observat. sur le Lampyris noctiluca, L.].

Swintox, A.-H. - Note su' les habitules de la Luciola italica, L., in Proceed. of the entomol. Soc. of London, ann. 1880, p. xxix.

Swiston, A.-II. - Insect Variety : Its Propuregation and Distribution, Londres, Paris et New-York; Cassell, Petter, Galpin et $\mathrm{C}^{\circ}$, p. 10I. [Liste d'Insectes phosphorescents, accompagnée de renseignements bibliographiques].

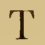

Targioni Tozzetri, Adolfo. - Come sia futto lorigann che fa lume nella Lucciola volante dell Italia centrale e come sieno composte le filne muscoluri in questo ed alti Insetti ed Artroporli, in Vlemor. della Soc. italiana di scienz. natur., Milan, ann. 1865, t. I, $n^{\circ} 8$, p. 28 , et 2 pl. - Tir. à part, Milan, I866. [Descript. des organes phosphorescents de la Luciola itrlicu, L., de ceux de la femelle et de la larve du Lampyris noctiluca, L., ete.].

Targions Tozzetti, Adolfo. - Sullorgano che fu lume melle Lurciole molanti ditulin. in Bull. della Soc. entomol. italiana, Florence. $2^{e}$ ann. (I870), p. I77; pl. I. 
fig. 8,14 his, 15, 16; et pl. II, fig. 1, 5 et 8 . |Lurciolı italica, L.].

Targions Tozzetri, Adolfo. - Note anatomiche intomo agli Insetti, in Bull. della Soc. entomol. italiana, Florence, $3^{\circ}$ ann. (1871), p. 386 , et pl. III. [Observat. anatomiques sur la larve du Lamp!n is noctilura. L., ete.|.

Templer, John. - Some Observations concerning GlowWo'ms, in Plilosoph. Transact. of the roy. Suc. of London, ann. 1671 , t. VI, n 72, p. 2177; $\mathrm{n}^{\circ} 78$, p. 3035; in Baddal, B. - Memoir. of the roy. Soc., being a new abridg. of the Philosoph. Transact., from 1665 to 1735 inclus., Londres, I738, t. 1, p. 313 et 335 ; et in Leske, N.-G.-Auserl. Abhandl. zur Naturgesch., prakt. Physik und Oekonomie aus den Philosoph. Transact. und Sammlung., Leipzig, I779, t. I, $1^{\text {re }}$ part., p. 87. [Lampyris noctiluca, L.].

Theobald. - Note sur la phosphorescence des Pyrophores, in Journ. of the asiatic Soc. of Bengal, Calcutta, I866, p. 73; et in Proceed. of the entomol. Soc. of London, ann. 1866, p. Xxvil.

Theodosius, Johannius-Baptistus. - Medicinales Epistolae. Bàle, N. Episcopius, 1553. Ep. 50, p. 305. [De Lampyridel.

Thylesius, Antonius. - De Araneola el Cicindela, in Liber de coloribus. Bàle, Froben, 1530, p. 536. [Lampyiris].

Thrlesius, Antonius. - De Ciciudela, in Dornavii Amphitheatr., t. I. [Lampyris]. 
Thidemax, Friedrich. - Physiologie de l'Homme, traduit de lallemand par A.-J.-L. Jourdax, Paris, 2 vol., 1831. t. II, p. 536. [Geienéralités sur les Insectes phosphorescents. - Tiedemann dit, entre autres (p. 5:38), que "parmi les Lépidoptères, le Pyjalis minor a labdomen faiblement lumineux, selon Brown »?]. [Ce Pypralis minor, que mentionne Patrick Brown (Op). cit., p. 431), n’est autre qu'un Coléoptère de la tribu des Lampyrides, probablement le Photuris (Lampyris) pensylunica, Degeer. (Voir Degeer. - Mém. pou. servir à l'Hist. des Insectes, t. IV (1774), p. 53)].

Tulesius. - Voir Macartary.

Topd, John-Tweedy. - An Inquiry respecting the mulure of the lum inous power of some of the Lamp!yridae, pasticularly of Lampyris splendidula, L., L. italica, L. et L. roctiluca, L.. in The quarterly Journ. of Scienc., Literat. and the Arts of the Roy. Institut., Londres, ann. 1824, t. XVIl, p. 269; ann. 1826, t. XX1, no 42, p. 241. [Lamprolisa splendidula, L., Luciola ilalica, L. et Lampyris noctiluca, L.].

[Extraitin The zoolog. Journ., Londres, ann. 1824, t. I, p. 274; in Froriep, L.-F. vos. - Notizen aus dem Gebiete der Natur- und Heilkunde, Weimar, ann. 1826 , t. $\mathrm{X}$ ', n $n^{0} 309$, p. 1 ; et in FÉrussac, A. DE. - Bull. univers. des scienc. natur. et de Géolugie, ann. 182 $\tau$, t. XII, p. 290].

Tournier, H. - Voir Tromelix.

Toussaint ue Cinarpextier. - Voir Cianpextier.

Townsend Guover. - Report of the Entomologist and Curator of the Museum. (Kep. of the Comm. of Agri- 
rulture for 1873). Washington, 1834, p. 152, fig. 1-10. Habits and Luminosity of Pyiophorus physsoderus, Germ. (fig. 3), compare dwith P. noctilucus, L. (fig. 4) and Photinus pyralis, L.

Townsend Glover. - A luminous Elaterid? Larea, in Psyche, Journ. of Entomol., published ly the Cambridge entomol. Club, Cambridge (Etats-Unis), lisit.

Trefrry. - Sur la non-phosphorescence du Fulyorra laternaria, L., in The Zoologist, Londres, ann. 1863, t. XXI.

Trevirants, Gottfried-Reinhold. - Ueber das Leurhten der Lampyris splendidula, L., in Treviranus, G.-R. et L.-C. - Vermischt. schrift. anat. und physiol. Inhalts, ann. 1816, t. I, p. 8i. [Lamproliza splendidula, L.].

Trevirands, Gottfried-Reinhold. - Biologie, oder Philosophie der lebenden Natur, für Naturforscher und Aer:te, Gottingue, Vandenhoeck, 6 vol., 1802-1822, t. V (1818). |Renseignem. nombreux sur les Insectes phosphorescents, avec lindication des travaux qui ont été publiés sur ce sujet, p. 96 ; et Note sur la phosphorescence des Pyrophores, p. 475].

Trimex, Roland. - On the occurrence of Astraptor illuminutor, Nurray or a closely allied species, near BuenosAyres, in Journ. of the Proceed. of the linn. Soc. of London, 1870, t. X, p. 503. [Lampyride?].

Tronelin, G. DE. - Note sur la phosphorescence du mâle du Lampyre, in Annal. de la Soc. entomol. de France, Paris, ann. 1871, bull. des séanc., p. cxxxvı. [Au sujet 
de la phosphorescence des màles de plusieurs espèces de Lampyres, des deux sexes de la Luciola lusitanica, Charp. et du Pholinus p!jralis, L., etc., différentes communications ont été faites par Alexandre LABotLbĖxe, Jean-Alphonse Bolsduval, Eugène Desmarest, Auguste Chevrolat, Hector Auzoux, Ernest Olivier, Al. Peratiallo et Paul Gandolpie ( ${ }^{0}$, p. Cxlitil); par Marie-Joseph Belox ( $d^{0}$, clxxiris); et par H. Tournier et Ch.-I. Rilei (d", p. Cxcis).

Turner. - Couleur de la lumière émise prar les Pyrophorus nortilucus, L., Photuris pensyleanicu, Degeer, et Pliotimus pyralis, L., in Psyche, Journ. of Entomol., publishel by the Cambridge entomol. Club, Cambridge (Etats-Unis), ann. 1882, t. III, p. 309.

\section{V}

Villaret, Foulques de. - Description d'une noucelle espece. du geure Lampyris (Lampyris Senclii, Villaret), in Annal. de la Soc. entomol. de France. Paris, ann. 1833, p. 352 , et pl. $\mathrm{XV}$, fig. 1 et a (màle), fig. 2 et b (larve). [Description de la larre du Lampyris Senclit, Villaret = Lampiolisa splendidula, L.].

V'iluers, Francois de. - Note sur la propriété phosphoresrente de petiles Fourmis joumes, in Innal, de la soc. entomol. de France, Paris, ann. I\& 12, bull. des séanc., p. xil1.

Viox, René. - Vers-luisants et Mouches phosphoriques, in Bull. de la Soc. linn. du Nord de la France, Amiens, ann. I872-I873, t. I, p. i2. 


\section{W}

Wagi, Gustave. - Sur les Larves des Lampyrides, in Motsciulsky, V. von. - Etudes entomol., Ilelsingfors, ann. 1856 , t. V, p. 40, av. fig.

Waillberg, P.-F. - Markvardig instinlit orh ljusutwecliling hos en svensli Myggart. (Ceroplatus sesioüdes, Whlbg.), in Ofvers. af kongl. Vetensk.-Akad. Forhandling., Stockholm, ann. 1848, t. V, p. I28; et in Stettiner entomol. Zeit., ann. 1819, t. X, p. 120. [Phosphorescence des larves et des nymphes du Ceroplatus sesioüles, Whlbg., Diptère de la famille des Mycétophilides].

[Analyse in Scinum, H.-R. - Bericht über Entomol., Berlin, ann, 1849, p. 101 (237)].

- Walles, George. - Observations on Ignis fatuus, in The entomol. Magaz., Londres., ann. 1832, t. I, p. 350. [Lampyris].

WaLker, Francis. - Discussion on the Luminosity of Fulgora candelaria, L., in The entomol. Magaz., ann. 1836, t. III, p. 45 et 105]. [Hotinus candelarius, L.] [Cet article, qui n'est pas signé, est peut-être de E. Newman. (Note de Hagen, H.-A. - Bibliotheca entomologica, Leipzig, IV. Engelmann, t. II (1863), p. 252)].

Walber, Richard. - Observations on the Cicindela volans or flying Glow-Worm, in Philosoph. Transact. of the roy. Soc. of London, ann. 1681, t. XV, $\mathrm{n}^{\circ} 167$, p. 811 , av. $1 \mathrm{pl}$;; in Baddan, B. - Memoir. of the roy. Soc., or a new abridg. of the Philosoph. Transact., Londres, 175, 


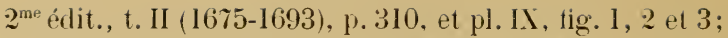
et in Acta Eruditor. Suppl., t. I, p.443;-in .ІаNGET, Bibl.,

t. II, $2^{\mathrm{me}}$ part., p. 568. [? Lampyris noctiluca, L. ơ]. Waterhouse, Charles-Owen. - Voir Eatox.

Wesmael, Constantin. - Note sur la Fulgore Lanterne, in Bull. de l'Acad. des scienc., des Lettr. et des Beaux-Arts de Belgique, Bruxelles, ann. 1838, t. IV, p. I36. [Wesmael soutient, d'après une observation faite par un naturaliste belge, Lisdex, que cet Hémiptère possède une propriėté phosphorescente].

[Extrait in L'Institut, Paris, ann. 183\%, t. V, n' 218, p. 259; in Annal. de la Soc. entomol. de France, Paris, ann. 1837, bull. des séanc., p. LXVII ; in FrorieP, L.-F. et R. vos. - Neue Notizen aus dem Gebiete der Natur- und Heilkunde, Weimar, ann. 1837, t. III, p. 231 ; et in Revue zoolog., par la Soc. cuviérienne, Paris, ann. 1838, t. I, p. 141].

Westfeld, Chr.-Friedrich-Gothard. - Voir Sigwart.

Westwood, John-Obadiah. - On the Family Fulgoridae, with a Wonograph of the genus Fulgora of Limaeus, in Transact. of the linn. Soc. of London, ann. 1839, t. XVIII, p. 133, et pl. XII, fig. 1-13. [Westwood nie la propriété phosphorescente de ces Hémiptères].

[Analyse in The philosoph. Magaz., Londres, ann. 1838, p. 93; in L'Institut, Paris, ann. 1838, t. V1, n²24, p. 66; in Annal. de la Soc. entomol. de France, Paris, ann. 1838, bull. des séanc., p. xxxrII ; et in Isis, Encyklop. Zeitschr., Leipzig, ann. 1843, t. VI, p. 434].

Westwood, John-Obadiah.-An Introduction to the modem classification of Insects, founded on the natural 
habits and corresponding oryanisation of the different families. Londres, Longman, t. I et II, 1839 et I810. [Renseignem. sur les Insectes phosphorescents].

Westwood, John-Obadiah. - Luminosity of Helobia brevicollis, Fabr., in Transact. of the entomol. Soc. of London, anı. I854, proceed., p. xxxiv; et in The Zoologist, Londres, ann. 1855, t. XIII, p. 4565. |Nebrin (Helobia) brevicollis, Fabr., Coléoptère de la famille des Carabides.-Westwoorl pense que l'apparence lumineuse de cet Insecte était due à un corps phosphorescent quelconque qui adlıérait à lui].

Westwood, John-Obadiah. - Note sur la phosphorescence de la Larve du Lampyris noctiluca, L., in Transact. of the entomol. Soc. of London, ann. I869, proceed., p. vı. Weyenbergh, H. - Eine leuchtende Kafer-Larve, in Horae Soc. entomol. rossicae, Saint-Pétershourg, ann. I876-1877, t. XII, p. 177, et pl. IV, B, fig. a.-e. [Larve de Téléphoride?].

[Analyse in Berthad, P. - Bericht über Entomol., Berlin, ann. 1875-1876, p. 198 (406)].

Weyenbergh, H. - Voir Napp.

White, W.-H. - The Glow-IVorm; the results of experiments in elucidation of a knowledge of its habits, in Iagaz. of Nat. Hist., Londres, ann. 1835, t. VIII, p. 623. [Lampyris noctiluca, L.].

Wied-Neuwied, Maximilian von. - Voir Neuwied.

Wielowiesski, Heinrich von. - Studien über die Lampyriden, in Zeitschr. für wissenschaftl. Zoologie, Leipzig, ann. 1882, t. XXXVII, p. 354 ; pl. XXIII et XXIV, 
fig. 1-46. Lampyris noctiluca, L. et Lamprohisa splendidula, L.]. [Travail très-important].

[Analyse in Mayer, P. et Giesbrecit, W. - Zoologisch. Jahresbericht der zoologisch. Station zu Neapel, Arthropoda, Leipzig, ann. 1882, p. 136; in The zoologic. Record, Londres, ann. 1882, Insecta, p. 78; in BERTKAU, P. - Bericht über Entomol., Berlin, ann. 1882. p. 224: in Biolog. Centralblatt, Erlangen, ann. 1883, t. III, ${ }^{\circ}$ 3, p. 69. (par Eusry, C.): in Archiv. de Zoolog. expériment. et générale, Paris, ann. 1883, p. xxx, (par Lacaze-Duthiers, H. DE); et in Nature, Londres et NewYork, 1883, t. XXVII, p. 231].

Wilson, Edward, - Voir Dale.

Wolcebius, Johann-Jacol. - Dissertatio de origine motus brutorum cum observationibus de Cicindela. Bàle, 1702. [Lamplris].

\section{Y}

Youna, C.-A. - On the spectrum of the light of Lampyris, in Journ. of the Soc. of Arts, Londres, ann. 1870; in Transact. of the entomol. Soc. of London, ann. 1870, proceed., p. Xvir ; et in The americ. Naturalist, Salem, ann. 1870, t. 1II, p. 615. [ R. Dubors dit (Les Elatérides lumineux, p. 106) que les recherches de Young ont été faites sur la llouche lumineuse commune du NewHampshire, qui est, selon toute probabilité, une espèce américaine du genre Photimus].

[Analyse in Brauer, F. - Bericht über Entomol., Berlin, ann. 1870, p. 5]. 


\section{SUPPLÉMENT}

\section{OBSERVATIONS ET RECTIFICATIONS.}

P. 20, 1. 6. - Le Pyralis minor, dont fait mention Patrick Brown (Op.cit., p. 131), n'est autre qu'un Coléoptère de la tribu des Lampyrides, probablement le Photuris (Lamplyris) pensylvanica, Degeer. (Voir l)egeer. - Mém. pour servir à l'Ilist. des Insectes, t. IV (I77.1), p. 53).

P. 16, 1. 20. - La Nymphe en question était celle d'un individu màle.

P. 19, 1. 19. - Ljouter : p. 137.

P. 65, 1. 19. - Ce travail d'Alexandre Laboulbène et de Charles Robın a été publié aussi in Robis, C. - Journ. de l'Anat. et de la Pliysiol., Paris, ann. 1873, t. IX, p. 593.

P. 69, 1. 17. - Ce travail de Ch. Loriquet est relatif au Pyrophorus noctilucus, L., et renferme, en outre, quelques renseignements sur la phosphorescence des Insectes. Il a été publié dans le Bulletin d'une Société dont j’ignore le nom, car je n’ai vu ce mémoire que sous for'me de feuillets détachés ( d'où ils proviennent.

P. 73, 1. 1\%. - Ajouter : [1. 112, et supprimer le point d'interrogation mis devant l'yrophores. 
P. 79, 1. 3 et 8 . - Au lieu de Owsiannikoff, lire : OWSJANNikow.

Dans la liste des travaux d'Hippolyte Lucas, donneje par Hagen dans sa Bibliotheca entomologica (Leipzig, W. Engelmann, t. I (I862), p. 498-506, et t. II (1863), p. 386-387), figure, au $\mathrm{n}^{\circ} 83$ de la p. 501 du t. I, un mémoire intitulé : "Note sur le Brachimus crepitans vivant de cadavres, et sur la Larve de la Lampyris noctiluca, Anm. Soc. ent. Fr., sér. 2., 1851, t. 9, p. 101 ». Cette indication nominative est erronée, car l'auteur de la note en question est G. Daumont et non Lucas, qui l'avait communiquée, au nom de G. Daumont, ̀̀ la Soc. entomol. de France; Hagen ayant indiqué par erreur le nom du présentateur de la note (Lucas) au lieu de celui de l'auteur (Daumont). De plus, il ne s'agit pas, dans cette communication, de la larve du Lampyris noctiluca, L., mais bien d'une nymphe d'un individu mâle de cette espèce, qui présentait deux points très-lumineux. Quant à l'indication bibliographique, elle n'est pas absolument exacte, et il faut lire, en employant les abréviations d'Hagen : Ann. Soc. ent. Fr., sér. 2., 1851, t. 9, Bull., p. 102.

Hagen a commis une erreur analogue en faisant figurer dans la liste des travaux de Louis Reiche (Bibliotheca entomologica, t. II, p. 67-69 et 390), au $n^{\circ} 16$ de la p. 67, une communication intitulée : "Note sur les propriétés lumineuses de Pyrophorus, Nyctophanes, et sur le bruit fait par les Passalus; Oecodoma cephalotes (Formica), Ann. Soc. ent. Fr., sér. 2, 1814, t. 2, Bull., p. 63-67 ". La première partie de cette communication [Nole sur les propriétés lumineuses de Pyirophorus, Nyctophanes, et sur le 
bruit fait par les Passalus] a pour auteur Emile Mocquerrs, et non Louis Reiche, qui n'était que le présentateur de cette note à la Soc. entomol. de France. Quant à la seconde partie [Oecorloma cephalotes (For'mica)], elle est bien en réalité de Louis Reiche.

\section{ADDENDA.}

Becquerel, Antoine-César. - Traité de Physique considérée dans ses rapports avec la chimie et les sciences naturelles. Paris, 2 vol., 1811. [Observat. sur la phosphorescence des Insectes].

Becquerel, Edmond-Alexandre. - La Lumière, ses causes el ses effets. Paris, 2 vol., 1867-1868. [Observat. sur la phosphorescence des Insectes].

Bourgeors, Jules. - Faune gallo-rhénane. Malacodermes. (En cours de publication dans la Revue d'Entomologie, Caen). [Renseignem. nombreux sur la bibliographie, la biologie et la phosphorescence des Lampyrides, p. 61 et suiv.].

Brischic. - Leuchtende Inipteren, in Kratz, Gustav. Entomol. Monatshlatter, Berlin, 18i6, p. 11. [Chironomus, Diptères de la famille des Chirononides].

Dubors, Raphä̈l. - Le laction de la lamière émise par. les itres vivants sur la rétine et sur les plarnes an 
gélatino-bromure (à propos d'une communication faite à la dernière séance par M. le professeur Pouchet), in Compt. rend. hebd. des séanc. de la Soc. de Biologie, Paris, ann. 1886, $\mathrm{n}^{\circ} \mathrm{I1}$, p. 130. [Observat. sur la lumière des Pyrophores].

Dubors, Raphaël. - Sur la luminosité des OEnfs d'Insectes, in Bull. de l'Associat. franç. pour l'Avancen. des Scienc., Congrès de Nancy, ann. I886, $\mathrm{I}^{\text {re }}$ part., p. I55. [Résumé très-court de son mémoire intitulé : De la fonction photogénique dans les OEufs du Lampyre, cité à la p. 49 de cette Bibliographie].

Duвors, Raphaël. - Recherches sur la fonction photogénique, in Compt. rend. hebd. des séanc. de l'Acad. des Scienc., Paris, ann. 1887, t. CIV, (séance du 23 mai I887), p. 1 156. [Observat. sur les Pyrophores et les Lampyrides, sur une espece de Luciole exotique, et sur des Podurides].

Euery, Carlo. - La Luce negli amori delle Lucciole, in Bull. della soc. entomol. italiana, Florence, $18^{\mathrm{e}}$ ann. (I886), p. 406.

Hasse, Erich. - Ein neuer Phengodes, in Entomol. Nachrichten, Berlin, $12^{\mathrm{e}}$ ann. (1886), $14^{\mathrm{e}}$ cah,, p. 218. [Renseignem. des plus sommaires sur les premiers états et sur la luminosité des deux sexes du Phengodes Hieronymi, Haase].

Jonston, Johann. - Historiae naturalis de Insectis libri III. ( 2 édit.), Amstelodami, schipper, I65i, ar. $28 \mathrm{pl}$. gravées, lib. I, cap. VIIl. [Pyrophores]. [La I ${ }^{\text {re }}$ élit. est de Francofurti ad Moen., H.-M. Merian, I653]. 
KoLBE, Hermann-J.-Ueber einige exolische Lepidopterenund Coleopteren-Larven. - 5. Eine Larve der Gattung Pyiophorus, in Entomol. Nachrichten, Berlin, $13^{\mathrm{e}}$ ann. (1887), $3^{\circ}$ cah., p. 36.

LioLbe, Ilermann-J. - Beobachtungen über Termiten und Leuchllafer (Lampyridce) im Caplande, nach brieflichen Mitheilungen des Herrn $D^{r}$ med. Fran: Bachmann, in Entomol. Nachrichten, Berlin, $13^{\circ}$ ann. (I887), 5 cah., p. 70.

Lucas, Hippolyte. - Description d'une Larve de Lampyride considérée comme devant appartenir an sexe femelle, dont l'Insecte parfait est encore inconnu, in Annal de la Soc. entomol. de France, Paris, ann. 1887, Résumé bull. des séanc., p. xxxv.

Meinert, Franz. - Gjennemborede Kindbakker hos Lampyris-og Drilus-Larverne, in Entomol. Tidskrift, Stockholm, ann. 1886, p. 194. [Ecrit en langue - Résun en français in même Bull.].

Menitzin. - Dans l'ouvrage de A.-H. Swinton intitulé : Insect Variely (voir Bibliograph., p. 92), je trouve à la p. 101 le renseignement bibliographique suivant, relatif à la luminosité du thorax et de l'abdomen du Chironomus tendens, Fabr., Diptère de la famille des Chironomides : Mexitzin, "Deutsche ent. Zeitschr. ", I875, p. 432. JJe n'ai pas vérifié l'exactitude de ce renseignement].

Murray, Andrew. - Larve du Photuris versicolor, Fabr., in Journ. of the Proceed. of the linn. Soc. of London, 
Zoology, ann. 1868, t. VIII, p. 71, et pl. I, fig. 9. |Photuris versicolor, Falır. = Phot. pensylvanica, Degeer].

Osten-Sacken, Charles-Robert von. - More about the luminous New-Zealand Larvae, in The Entomol. monthl. Magaz., Londres, ann. 1887, $n^{\circ}: 27$. |Voir, à ce sujet, le mémoire d'Hudson (Bibliograph., p. 61) et celui d'Osten-Sacken (d ${ }^{\circ}$, p. '78)].

Packard, A.-S. jun. - Guide to the study of Insects, av. $15 \mathrm{pl}$. et de très-nombr. fig., $8^{\ominus}$ élit., New-York, Henry Holt et $\mathrm{C}^{\circ}$; Boston, Estes et Lauriat; 1883, p. 462, 465-467, et 533. [Observat. sur des Insectes phosphorescents (Pyrophorus, Melanactes, Lampyrides, Fulgorides)].

PaCkARD, A.-S. jun. - Luminous Organs of mexican Cucuyo. [Je n'ai pu trouver d'indications bibliographiques relatives à ce mémoire qui, peut-être, a paru in The americ. Naturalist].

Secchi, Angelo. - Nouvelles Observations sur les lumières phosphorescentes animales. (Extrait d'une lettre en date du 24 juillet 1872, adressée à l'Académie des Sciences), in Ann. des Scienc. natur., Zoolog., ann. 1872, $5^{\mathrm{e}}$ sér., t. XVI, art. 9. [Cette communication, qui a paru d'abord in Compt. rend. hebd. des séanc. de l'Acad. des Scienc., Paris, ann. 1872 , t. LXXV, p. 321 , est suivie de Remarques par Armand de Quatrefafes de BrÉau, p. 322]. [Observat. sur la phosphorescence d'Insectes]. 
Dans lẹ Bericht über die wissenschaftlichen Leistungen im Gebiete der Entomologie pendant l'année 1880, de P. Bertkau, je trouve à la p. 228 le renseignement suivant, malheureusement incomplet, dont je n'ai pas vérifié l'exactitude: Une larve de Coléoptère fortement lumineuse, rapportée avec doute au genre Melanactes, est figurée in The americ. Naturalist, 1880, p. 202. 



\section{TABLE ALPHABÉTIQUE DES NONS D'AUTEURS.}

Afzelius, $\mathrm{A}$.

Aldrovande, $\mathrm{U}$.

Allen, B.

Allman, G.-J.

Anghiera, P. Martire d'.

Anonyme.

Aristote.

Arnold, C.

Aubert.

Audouin. J.-V.

Austin .

Auzoux, H.

Azara, F. de.

Bach, M.

Bacon, F.

Bacouni, A. de.

Baron.

Barrère.

Bartholin, T.

Bates.

Bates, H. $-1 \mathrm{I}$.

Beach, A.-E. [Editeur].

Beauvois, A.-M.-F.-J. Palisot de.

Becker, J. von.

Becker, J .-J.-M.

Beckerheim.
Becquerel, A.-C. [Ardenda]. Becquerel, E.-A. [Addenda]. Bellesme, Jousset de.

Belon, M.-J.

Bernoulli, C.

Berthold, A. $-\Lambda$.

Beske.

Béthune, C.-J.-S.

Blanchard, E.

Blanchet, R.

Blesson, L.

Boiscluval, J .-A.

Boll, E.-F.-A.

Bondaroy, A.-D. Fougeroux de.

Bonpland, A.

Bottoni, II.

Bourgeois, J. [Addenda].

Bowles, G.-H.

Bowring, J . -C.

Branner, J .-C.

Brischke. [Addenda].

Brown, P. [Bibliogr. et

Observ. et Rectific.].

Brugnatelli, L.-G.

Bıuguière, J.-G.

Brullé, A.

Burmeister, H.-C.-C. 
Burnett, II . -I.

Camerarius, J.-R.

Cameron, J .

Candèze, E.

Carpenter, W.-B.

Carradori, G.

Carrara, M.

Carus, C.-G.

Castelnau, de. (F.-L. de Laporte).

Chabrillac, F.

Champion, G.-C.

Charpentier, Toussaint de.

Chevrolat, $\Lambda$.

Clark.

Columna, F.-L.

Conroy.

Couper.

Curtis, J.

Dale, J.-C.

Darwin, C.

Daumont, G.

Davy, H.

Degeer, C.

Desmarest, E.

Dieckhoff, L.-A.

Dollfus, A.

Dortous de Mairan, J.-J.

Doubleday, E.
Dubois, R. |Bibliogr it Aidenda].

Dufour, L.

Dutertre.

Eaton, A.-E.

Edwards, H.

Edwards, H. Milne-.

Ehrenberg, C.-G.

Enery, C. |Bibliogr. et Addenda].

Emmert.

Enell, H.

Erichson, W.-F.

Evans, W.-T.

Faille, J.-M. Baart de la.

Fairmaire, L.

Fennell, J.

Ferchault de Réaumur, R.-A.

Fermin, P.

For'ster, J.-G.-A.

Fougeroux de Bondaroy, A.-D.

Foulques de Villaret.

Fry, A.

Gadeau de Kerville, $\mathrm{H}$.

Gandolphe, P.

George, H. jun.

Gernez. 
Gimmerthal, B.- $\Lambda$.

Girard, NI.

Glover, Townsend.

Gorham, H.-S.

Gosse, P.-H.

Gounelle, E.

Goureau.

Greenwool Penny, R.

Grew, N.

Gronov, L.-T.

Grotthuss.

Guéneau de Montbeillard, P.

Guenther, J.

Guilding, L.

Haase, E. [Bibliogr. et Addenda].

Hagen, H.-A.

Hamlet.

Hancock, J.

Harting, P.

Heinemann, C.

Heinrich.

Helbig.

Henderson, G.

Henning, J.-F.

Henslow, (i.

Hermanas, de Dos.

Hermbstaedt, S.-F.

Heward, R.

Hoeven, J. van der.
Hoffmansegg, J.-C. von.

Hudson, G.-V.

Hulme, N.

Humboldt, F.-H.-A. von.

Illiger, J.-C.-IV.

Imhoff, L.

Jenner, J.-H.-A.

Jennings, J.

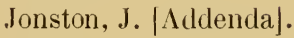

Joseph, G.

Jousset de Bellesme.

Kaffer.

Kaiser, W.

Kawall, $\mathrm{H}$.

Kerville, H. Gadeau de.

King, H.-S.

King, V.-O.

Kirby, W.

Kircher, A.

Koelliker, A.

Kolbe, H.-J. [Addenda].

Laboulbène, A. [Bibliogr. et

Observ. et Rectific.].

Lacaze-Duthiers, F.-J.-H.de.

Lacordaire, J.-T.

Lamarck, J.-B.-P.-A. de

Monet de. 
Laporte, F.-L. de. (de Castelnau).

Lartigue, $\mathrm{H}$.

Latreille, P.-A.

Le Conte, J.-L.

Lewis, G.

Leydig, F.

Lindemann, C.

Linden.

Linné, C. von.

Loche, F. Mouxy de.

Loriquet, C. [Biblingr. et Observ. et Rectific.|.

Lucas, H. [Addenda].

Luce.

Nacaire, I.-F.

Macartney, J.

Mac Lachlan, R.

Mac Laurin, IV.

Naille, $\Lambda$.

Main.

Main, J.

Mairan, J.-J. Dortous de.

Martin.

Martire d'Anghiera, P.

Nartius, C.-F.-P. von.

Natteucci, C.

Maurer, F.

Meinert, F. [Addenda].

Melchior, J.-A.
Meldola.

Menitzin. [Addenda].

Merian, M.-S.

Milne-Edwards, H.

Nocquerys, E.

Monet de Lamarck, J.-B.-

P.-A. de.

Montbeillard,P.Guéneau de.

Iontrouzier.

Morren, C.-F.-A.

Morris.

Noufet, T.

Ioufflet, A.

Mouxy de Loche, F.

Ineller, P.-IV.-J.

Mulsant, E.

Nuralto, J. von.

Nurray, A. [Bibliogr. et

Addenda].

Murray, J.

Napp, R.

Naudin, C.-I.

Newall, R.-..

Newman.

Newman, E.

Neuw ied, M. ron Wied-.

Nerrport, $G$.

Nieremberg, J.-E.

Nollet, J.-A.

Norirood. 
Olivier, 1.-(i.

Olivier, E.

Osten-Sacken, C.-R. von.

[Bi' liogre. et Addenrla].

Oviedo y Valdes, G.-F. de.

Owsjannik»w, P.

Packard, A...s. jun. |N,ldenda].

Palisot de Beaurois, A.-MI.F.-J.

Pallas, I.-S.

Parfitt, E.

Parzudaki.

Pasteur, L.

Penny, R. Greenwood.

Peragallo, A.

Percheron, A.-R.

Perkins, G.-A.

Perty, M.

Peters, IV.-C.-H.

Pflüger, E.-F.-IV.

Phipson, T.-L.

Pickman Mann, B.

Plinius secundus, C.

Poujade, G.-A.

Pryer, W.-B.

Quatrefages de Bréau, A. de. [Addenda].
Radziszewski.

Ramsilen.

Ray, J.

Razoumowsky, G. von.

Réaumur, R.-A. Ferchault de.

Recluz.

Regnard, P.

Reiche, L.

Reinlıarit, J.-T.

Revelière, E.

Rey, C:

Riley, C.-I.

Rivinus, Q.-S.-F.

Robert, E.

Robin, C. [Bibliogr. et Observ. et Rectific.].

Robineau-Desvoidy, A.-J.-B.

Rochefort, C. de.

Rogerson, IV.

Rouzet, J.-H.

Sang, J.

Saunder's.

Scaliger, J.-C.

schioedte, J.-C.

Schmid, C. -A.

Schneider, IV.-G.

Schnetzler, J.-B.

Schultze, M.-S.

Secchi, A. [Addenda]. 
Sells, IV.

Severn, H.-A.

Sharp.

Sluaw, J.

Shufeldt, R.-IV.

Sigwart, G.-F.

sloane, H.

Smith, J.

Soemmerring, S.-T. von.

sorg, F.-L.-A.-IV.

Spallanzani, L.

Spence, IV.

Spiller.

Spinola, M.

spix, J.-B. von.

Stedman, J.-G.

Steinheil, E.

stillman, IV.-J.

Stollwerck, F.

Strickland, H.-E.

Stubbes.

Swammerdamm, J.

Swinton, A.-H.

Targioni Tozzetti, A.

Templer, J.

Theobald.

Theodosius, J.-B.

Thylesius, A.

Tiedemann, F.

Tilesius.
Todd, J.-T.

Tournier, H.

Toussaint de Charpentier.

Townsend Glover.

Treffry.

Treviranus, G.-R.

Trimen, R.

Tromelin, G. de.

Turner.

Villaret, Foulques de.

Villiers, F. de.

Vion, R.

Waga, G.

Wahlberg, P.-F.

Wailes, G.

Walker, F.

Waller, R.

Waterhouse, C.-O.

Wesmael, C.

Westfeld, C.-F.-G.

Westwood, J.-O.

Weyenbergh, $\mathrm{H}$.

White, W.-H.

IVied-Neuwied, M. von.

Wielowiejski, H. von.

Vilson, E.

Wollebius, J.-J.

Young, C.-A. 


\section{TABLE NLPHABÉTIQUE DES NOMS D'AUTEURS}

PAR GROUPE D'INSECTES PHOSPHORESCENTS.

\section{GÉNÉRALITÉS ET OBSERVATIONS DIVERSES.}

Anghiera, P. Martire d'.

Azara, F. de.

Bach, II.

Bacon, F.

Barrère.

Bartholin, T.

Bates.

Beach, A.-E. [Editeur].

Beauvois, A.-M.-F.-J. Palisot de.

Beckerheim.

Becquerel, A.-C. [Addenda]. Becquerel, E.- $\Lambda$. [Addenda].

Berthold, A.-A.

Brown, P.

Brugnatelli, L.-G.

Brullé, A.

Burmeister, H.-C.-C.

Cameron, J.

Carpenter, W.-B.

Castelnau, de. (F.-L. de Laporte).

Clark.

Darwin, C.
Dortous de Mairan, J.-J.

Dutertre.

Edwards, H. Milne-.

Ehrenberg, C.-G.

Faille, J.-M.-Baart de la.

Gadeau de Kierville, $H$.

Gronov, L.-T.

Guilding, L.

Hamlet.

Harting, P.

Imhoff, L.

King, V.-O.

Kirby, W.

Lacaze-Duthiers, F.-J.-H.de.

Lacordaire, J.-T.

Laporte, F.-L. de. (de Castelnau).

Latreille, P.-A.

Linné, C. von.

Loriquet, C. [Bibliogr. et

Observ, et Rectific.].

Macartney, J.

Nac Lachlan, R.

Mairan, J.-J. Dortous de. 
Martire d'Anghiera, P.

Martius, C.-F.-P. von.

Nelchior, J.-A.

Milne-Edwards, $\mathrm{H}$.

Nontrouzier.

Napp, R.

Nieremberg, J.-E.

Norwood.

Oviedo y Valdes, G.-F. de. Palisot de Beauruis, M.-M.F.-J.

Percheron, A.-R.

Perty, M.

Pflüger, E.-F.-IV.

Phipson, T.-L.

Pryer, W.-B.

Quatrefages de Bréau, $\Lambda$. de. [Addenda].
Rarlziszewski.

Ray, J.

Reinhardt, J.-T.

Rivinus, Q.-S.-F.

Rochefort, C. de.

Saunder's.

Secchi, A. [Addenda].

Spence, IV.

Spix, J.-B. von.

Stubbes.

Swinton, A.-H.

Tiedemann, F.

Tilesius.

Treviranus, G.-R.

Vion, R.

Westwood, J.-0.

Weyenbergh, II.

\section{COLÉOPTĖRES.}

Elatérides.

Aldrovande, U.

Anonyme.

Aubert.

Baron.

Béthune, C.-J.-S.

Blanchard, E.

Boll, E.-F.-A.

Bondaroy, A.-D. Fougeroux - de.
Bonpland, A.

Bowles, G.-H.

Burnett, IV.-I.

Candèze, E.

Carpenter, IV.-B.

Couper.

Curtis, J.

Degeer, C. 
Dubois, R. [Biblingr. of Addenda].

Elirenberg, C.-G.

Erichson, W.-F.

Fougeroux de Bondaroy, A.-I).

Gernez.

Girard, II.

Glover, Townsent.

Gosse, P.-H.

Heinemann, C.

Hermanas, de Dos.

Heward, R.

Hoeven, J. van der.

Ilumboldt, F.-II.-A. von.

Illiger, J.-C.-IV.

Jonston, J. [Addenda].

Loelliker, $\Lambda$.

Kiolbe, H.-J. [Addenda].

Laboulbène, A. [Bihliogr. ct

Observ, et Rectific.].

Lacordaire, J.-T.

Loriquet, C. [Bib]liogr. et

Olsserv. et Rectific.].

Hacartney, J.
Mocquerys, E.

Morris.

Moufet, $\mathrm{T}$.

Osten-Sacken, C.-R. von.

Packard, A.-S. jun. [Addenda].

Pasteur, L.

Perkins, G.-A.

Phipson, T.-L.

Pickman Mann, B.

Ramsden.

Robin, C. [Bibliogr. et Ubserv. et Rectific.].

Schioedte, J.-C.

Sells, II.

Sliufeldt, R.-II.

Sloane, H.

Smith, J.

Steinheil, E.

stollwerck, F.

Stubbes.

Theobald.

Townsend Glover.

Treviranus, G.-R.

Turner.

Malacodermes.

Alelrovande, $\mathrm{U}$.

Allen, B.

Anonyne.
Aristote.

Arnold, C.

Aulouin, J.-V. 
Austin.

Auzoux, H.

Bach, N.

Bacouni, 1. de.

Becker, J. von.

Bellesme, Jousset de.

Belon, II.-J.

Bernoulli, C.

Berthold, A.-A.

Béthune, C.-J.-S.

Blanclet, R.

Blesson, L.

Boisduval, J.-A.

Bottoni, I).

Bourgeois, J. [Addenda].

Brown, P. [Biblingr. et Observ. et Rectific.].

Brugnatelli, L.-Cx.

Bruguière, J.-G.

Camerarius, J.-R.

Candèze, E.

Carpenter, W.-B.

Carradori, G.

Carrara, II.

Carus, C.-G.

Charpentier, Toussaint de.

Chevrolat, A.

Columna, F.-L.

Conroy.

Couper.

Dale, J.-C.
Daumont, G.

Davy, $\mathrm{H}$.

Degeer, C.

Desmarest, E.

Dieckhoff, L. $-\Lambda$.

Dollfus, A.

Dubois, R. [Bibliogr. et Addenda].

Dufour, L.

Eaton, A.-E.

Ehrenberg, C.-G.

Emery, C. [Billiogr. et Addencla].

Emmert.

Enell, H.

Erichson, W.-F.

Fairmaire, L.

Fennell, J.

Forster, J.-G.- $\Lambda$.

Foulques de Villaret.

Fry, A.

Gandolphe, P.

Girard, $\mathrm{N}$.

Glover, Townsend.

Gorham, H.-S.

Gosse, P.-H.

Goureau.

Greenwood Penny, R.

Grotthuss.

Guéneau de Montbeillard, P. Guenther, J. 
Haase, E. [Biblingr. et A.ldenda).

Heinrich.

Helbig.

Henderson, G.

Henning, J.-F.

IIenslow, G.

Hermbstaedt, s.-F.

Heward, R.

IIulme, N.

Illiger, J.-C.-IV.

Jenner, J.-H.-A.

Jennings, J.

Joseph, G.

Jousset de Bellesme.

Kaiser, W.

Kawall, $\mathrm{H}$.

King, H.-S.

Kircher, A.

Koelliker, $\Lambda$.

Kolbe, H.-J. [Addenda].

Laboulbène, A.

Lacordaire, J.-T.

Lartigue, $\mathrm{H}$.

Latreille, P.-A.

Le Conte, J.-L.

Leydig, F.

Lindemann, C.

Loche, F. Mouxy de.

Lucas, H. [Addenila].

Luce.
Masaire, I.-F.

Macartney, J.

Inc Lachlan, R.

Mac Laurin, WV.

Maille, $\Lambda$.

Main, J.

Martin.

Mattencei, C.

Maurer, F.

Meinert, F. [Addenda].

Meldola.

Mocquerys, E.

Mon theillard, P'. Guéneau de.

Morren, C.-F.-A.

Morris.

Mouxy de Loche, F.

Ineller, P.-IV.-J.

Mulsant, E.

Muralto, J. von.

Iurray, A. [Bibliogre el Addenda].

Murray, J.

Naudin, C.-V.

Newall, R.-S.

Newport, G.

Nollet, J.-A.

Olivier, E.

Osten-Sacken, C.-R. von.

Owsjannikow, P.

Packard, A.-S. jun. [Addenda]. 
Parfitt, E.

Penny, R. Greenwood.

Peragallo, $\Lambda$.

Peters, W.-C.-H.

Phipson, T.-L.

Plinius Secundus, C.

Poujade, G.-A.

Ray, J.

Razoumowsky, G. ron.

Recluz.

Regnard, P.

Reiche, L.

Revelière, E.

Rey, C.

Riley, C. $-\mathrm{V}$.

Robert, E.

Rogerson, W.

Sang, J.

Scaliger, J.-C.

Schmil, C.-A.

Schneider, W.-G.

Schnetzler, J.-B.

Schultze, M.-S.

Severn, H.-A.

Sharp.

Shaw, J.

Sigwart, G.-F.

Soemmerring, S.-T. von.

sorg, F.-L.-A.-IV.

Spallanzani, L.

Spiller.
Stillman, W.-J.

Strickland, H.-E.

Swammerdamm, J.

Swinton, A.-H.

Targioni Tozzetti, $\Lambda$.

Templer, J.

Theodosius, J.-B.

Thylesius, $\mathrm{A}$.

Todd, J.-T.

Tournier, H.

Toussaint de Charpentier.

Townsend Glover.

Treviranus, G.-R.

Trimen, R.

Tromelin, G. de.

Turner.

Villaret, Foulques de.

Waga, G.

Wailes, G.

Waller, R.

Westfeld, C.-F.-G.

Westwood, J.-O.

Weyenhergh, H.

White, W.-II.

Wielowiejski, H. ron.

Wilson, E.

Wollebius, J.-J.

Young, C.-A. 
Carabides.

Parzudaki.

Reiche, L.

Rouzet, J.-II.

Westwood, J.-O.

Staphylinides.

George, H. jun. | Peragallo, A.

\section{Pausides.}

Afzelius, A.

Buprestides.

Latreille, P.-A.

Ténébrionides.

Lamarck, J.-B.-P.-A. de Nonet de.

Cérambycides.

Chevrolat, A.

\section{HÉMIPTËRES.}

Fulgorides.

(Observations affirmatives et négatives).

Bates, H.-W.

Becker, J.-J.-II.

Beske.

Bowring, J.-C.

Branner, J.-C.
Carpenter, W.-B.

Chabrillac, F.

Champion, r.-C.

Degeer, C.

Doubleday, E. 
Edwards, H.

Evans, IV.-T.

Ferchault de Réaumur, R.-A.

Fermin. P.

Girard, M.

Gounelle, E.

Grew, $x$.

HIagen, H.-A.

Hancock, J.

Hoffmansegg, J.-C. ron .

Kaffer.

Lacordaire, J.-T .

Linden.

Linne, C. von.

Jerian, II.-s.

Nouftlet, $A$.

Neuwied, II. von Wied-.
Newman.

Newman, E.

Olivier, A.-G.

Packard, A.-S. jun. [Addenda].

Pryer, IV.-B.

Réaumul, R.-A. Ferchault de.

Smith, J .

Spence, II.

Spinola, Mi.

Stednıan, J .-G.

Treffry.

Walker, F.

Wesmael, C.

Westrood, J.-O.

Wied-Neuwied, M. ron.

\section{ORTHOPTËRES.}

Podurides.
Allman, G.-J.
I Dubois, R. [Addenda].

Ephémérides.

Eaton, A.-E.

Hagen, H.-A.

Lewis, G.

Waterhouse, C.-O.

\section{DIPTËRES.}

Mycétophilides.

Hudson, G.-Y .

Osten-Sacken, C.-R. ron. [Bibliogr. et Addenda]. Wahlberg, P.-F. 
Chironomides.

Brischke. [Addenda].

Menitzin. [Addenda].

Osten-Sacken, C.-R. von.

Culicides.

Pallas, P.-S.

Tipulides.

Main .

Muscides.

Robineau-Desvoidy, A.-J.-B.

\section{LÉPIDOPTËRES.}

Agrotides.

Gimmerthal, B.-A.

Hadénides.

Boisduval, J.-A.

HYMENOPTERES.

Formicides.

Villiers, F. de. 


\section{TABLE ALPHABÉTIQUE DES NOIS D AUTEURS}

PAR DATE DE PUBLICATION DE LEURS TRATAUX

SUR LES INSECTES PIIOSPIIORESCENTS.

\section{ANTIQUITÉ.}

Aristote.

I Plinius Secundus, C.

$$
1501-1550 .
$$

Anghiera, P. Martire d'. | Oviedo y Valdes, G.-F. de.

Thylesius, A.

$$
1551-1600 .
$$

Scaliger, J.-C.

I Theodosius, J.-B.

$$
\text { 1601-1650. }
$$

Aldrorande, U.

Bacon, F.

Bartholin, T.

Camerarius, J.-R.
Columna, F.-L.

Kircher, A.

Iloufet, T.

Nieremberg, J.-E.
Bottoni, D.

Dutertre.

Grew, N.

Jonston, J . [Addenda].

Muralto, J. ron.

Norwood.

\section{1-1700.}

Rivinus, Q.-S.-F.

Rochefort, C. de.

Stubbes.

Templer, J.

Waller, R. 


\section{$1701-1725$.}

Allen, B.

Iortous de Mairan, J.-J.

Guenther, J.

Henning, J.-F .
Merian, M.-S.

Ray, J.

Sloane, II.

Wollebius, J.-J.

\section{$1726-1750$.}

Degeer, C.

Linné, C. von.

Melchior, J.-A.

Nollet, J.-A.
Réaumur, R.-A. Ferchault de.

Swammerdamm, J.

\section{$1751-1775$.}

Bondaroy, A.-I). Fougeroux de.

Brown, P.

Degeer, C.

Fermin, P.
Gronov, L.-T.

Linné, C. von.

Sigwart, G.-F.

Westfeld, C.-F.-G.

\section{6-1800.}

Afzelius, A.

Bacouni, A. de.

Beckerheim.

Brugnatelli, L.-G.

Bruguière, J.-G.

Carradori, G.

Forster, J.-G.-A.

Guéneau de Montbeillard, P.
Hulme, N.

Luce.

Olivier, A.-G.

Pallas, P.-S.

Razoumowsky, G. von.

Soemmerring, S.-T. von.

Spallanzani, L. 


\section{1-1810.}

Azara, F. de.

Beauvois, A.-M.-F.-J. Palisot de.

Bernoulli, C.

Carrallori, G.

Davy, $\mathrm{H}$.

Hellig.

Hermbstaedt, S.-F.

Hoffmansegg, J.-C. von.

Hulme, N.

Illiger, J.-C.-W.
Lamarck, J.-B.-P.-A. de Monet de.

Loche, F. Mouxy de.

Macartney, J.

Iueller, P.-IV.-J.

Palisot de Beauvois, 1.-II.F.-J.

Schmid, C.-A.

Sorg, F.-L.-A.-II.

Stedman, J.-G.

\section{1-1820.}

Bonpland, A.

Brugnatelli, L.-G.

Carradori, G.

Heinrich.

Humbolelt, F.-H.-A. von.

Kirby, IV.
Latreille, P.-A.

Neuwied, M. von Wied.

Spence, II.

Tilesius.

Treviranus, G.-R.

\section{1-1830.}

Anonyme.

Berthold, A.-A.

Carus, C.-G.

Charpentier, Toussaint de.

Curtis, J.

Dufour, L.

Faille, J.-M.-Baart de la.
Gimmerthal, B.-A.

Grotthuss.

Lacordaire, J.-T.

Latreille, P.-A.

Nacaire, I.-F.

Maille, $\mathrm{A}$.

Martius, C.-F.-P. von. 
Nurray, J.

Perty, M.

Recluz.

Rogerson, W. spix, J.-B. von.

Todd, J.-T.

Toussaint de Charpentier.

\section{1-1840.}

Anonyme.

Audouin, J.-V.

Barrère.

Blesson, L.

Boisduval, J.-A.

Brullé, A.

Burmeister, H.-C.-C.

Carrara, M.

Castelnau, de. (F.-I. de Laporte).

Chevrolat, A.

Dale, J.-C.

Doubleday, E.

Ehrenberg, C.-C.

Fennell, J.

Foulques de Villaret.

Guilding, L.

Hancock, J.

Jennings, J.
Lacordaire, J.-T.

Laporte, F.-L. de. (de Castelnau).

Linden.

Main.

Main, J.

Newman, E.

Percheron, A.-R.

Sells, IV.

Spinola, M.

Strickland, H.-E.

Tiedemann, F.

Villaret, Foulques de.

Wailes, G.

Walker, F.

Wesmael, C.

Westwood, J.-O.

White, W.-H.

IVilson, E.

\section{1-1850.}

Allman, G.-J.

Becker, J.-J.-M.

Becquerel, A.-C. [Arddenda].
Beske.

Bowring, J.-C.

Burnett, IV.-I. 
Carpenter, W.-B.

Dieckhoff, L.-..

Edwards, H.

Erichson, IV.-F.

Gosse, P.-H.

Goureau.

Henderson, G.

Heward, R.

Kaffer.

Natteucci, C.

Hocquerys, E.
Morren, C.-F.-A.

Parzudaki.

Peters, W.-C.-II.

Reiche, L.

Robert, E.

Robineau-Desvoidy, A.-J.-B.

Rouzet, J.-H .

Spence, IV.

spinola, M.

Villier's, F. de.

Wahlberg, P.-F.

\section{$1851-1855$.}

Daumont, G.

George, II. jun.

Hagen, H.-1.

Harting, P.

Hoeven, J. van der.

Humboldt, F.-H.-A. von.
Josepl, G.

Lacaze-Duthicrs, F.-J.-II.de.

Reinhardt, J.-T.

Schnetzler, J.-B.

Westwood, J.-O.

\section{6-1860.}

Bach, II.

Blanchet, R.

Boll, E.-F.-A.

Chabrillac, F.

Imhoff, L.

Koelliker, A.

Leydig, F.
Montrouzier.

Nulsant, E.

Newport, G.

Phipson, T.-L.

Revelière, E.

Schneider, W.-G.

Waga, G. 


\section{1-186i).}

Bates.

Bates, H.-W:

Bethune, C.-J.-S.

Blanchard, E.

Candèze, E.

Carus, C.-G.

Clark.

Couper.

Edwards, H. Milne-.

Evans, W.-T.

Fry, A.

Gernez.

Hagen, H.-A.

Hamlet.

Laboulbène, $\Lambda$.

Lindemann, C.

Mac Lachlan, R.
Milne-Eiwards, H.

Morris.

Moufflet, $\Lambda$.

Iulsant, E.

Newman.

Osten-Sacken, C.-R. von. Orvjannikow, P.

Pasteur, L.

Peragallo, A.

Phipson, T.-L.

Reiche, L.

Saunders.

Schultze, M.-S.

Smith, J.

Targioni Tozzetti, A.

Treffry.

\section{6-1870.}

Becker, J. von .

Becquerel, E.-A. [Addenda].

Candèze, E.

Fairmaire, L.

Girard, M.

Kawall, H.

Murray, A. [Bibliogr. et $\mathrm{Ad}$ denda].

Naudin, C.-V.
Owsjannikow, P.

Perkins, G.-A.

Schioedte, J.-C.

Smith, J.

Targioni Tozzetti, A.

Theobald.

Trimen, R.

Westwood, J.-O.

Young, C.-A. 


\section{1-1875.}

Auzoux, H.

Baron .

Beach, A.-E. [Editeur].

Bellesme, Jousset de.

Belon, M.-J.

Boisduval, J.-A.

Burmeister, H.-C.'C.

Chevrolat, A.

Desmarest, E.

Eaton, A.-E.

Emmert.

Gandolphe, P.

Girard, $\mathrm{N}$.

Glover, Townsenil.

Hagen, H.-A.

Heinemann, C.

Hermanas, de Dos.

Jousset de Bellesine.
Laboulbène, $\mathrm{A}$.

Lartigue, $\mathrm{H}$.

Menitzin. [Arldenda].

Olivier, E.

Peragallo, $A$.

Pflüger, E.-F.-IV.

Pickman Mann, B.

Quatrefages cle Bréau, . . dr

[Addenda].

Riley, C.-I.

Robin, C.

Secchi, A. [Adddenda].

Smith, J.

Targioni Tozzetti, $\perp$.

Tournier, $\mathrm{H}$.

Tornsend Glover.

Tromelin, G. de.

Vion, R.

\section{6-1880.}

Austin .

Bellesme, Jousset de.

Brischlie. [Addenda].

Eaton, A.-E.

Gorlıam, H.-S.

Gosse, P.-H.

Greenwood Penny, R.

Henslow, G.

Jousset de Bellesme.
King, II.-S.

King, V.-O.

Le Conte, J.-L.

Mac Lachlan, R.

Nac Laurin, W.

Martin.

Napp, R.

Newall, R.-S.

Osten-Sacken, C.-R. von. 
Parfitt, E.

Penny, R. Greenwood.

Pryer, W.-B.

Radziszewski.

Ramsden.
Sharp.

Shaw, J.

Sivinton, 1.-II.

IVeyenbergh, H.

\section{1-1885.}

Arnold, C.

Aubert.

Bourgeois, J. [Adelenda].

Bowles, G.-H.

Bramner, J.-C.

Champion, G.-C.

Conroy .

Dollfus, $\Lambda$.

Dubois, R.

Eaton, A.-E.

Emery, C.

Enell, H.

Gadeau de kierville, $\boldsymbol{H}$.

Girard, 11 .

Haase, E.

Jenner, J.-H.-A.

Kaiser, W.

Laboulbène, A.
Lewis, G.

Meldola.

Olivier, E.

Packard, A.-S. jun. [Adden(la].

Poujade, G.-A.

Regnari, P.

Rey, C.

Sang, J.

Severn, II.-A.

Shufeldt, R.-IV.

Spiller.

Stillman, W.-J.

Stollwerck, F.

Turner.

Waterhouse, C.-O.

Wielowiejski, H. von.

\section{6-1887.}

Dubois, R. [Bibliogr. et Addenda].

Emery, C. [Addenda]. Gounelle, E.

Haase, E. [Addendal. ud son, G.-V.
Kolbe, H.-J. [Addentia].

Lucas, H. [Addenda].

Meinert, F. [Addenda].

Olivier, E.

Osten-Sacken, C.-R. von. ¡Biblingr. et Aildenda]. 


\section{OBSERVATIONS IIVERSES}

RELATIVES A LA TABLE ALPHABÉTIQLe dES NOMS D'AUTELRS

PAR DATE DE PUblCation de leurs tRavaux

SUR LES INSECTES PIIOSPHORESCENTS.

Cameron, J. [La date de publication de son ouvrage, cité à la p. 43 , m'est inconnue].

Darwin, C. [J'ignore à quelle époque a été publié, pour la première fois, l'ouvrage de cet auteur renfermant des observations sur des Insectes phosphorescents].

Loriquet, C. [La date de publication de son mémoire, cité aux p. 69 et 101, m’est inconnue].

Maurer, F. [La date de publication de son mémoire, cité à la p. 72, m'est inconnue].

Packard, A.-S. jun. [La date de publication de son mémoire intitulé : Luminous Organs of mexican Cucuyo, cité à la p. 106, m’est inconnue].

Steinheil, E. [La date de publication de son mémoire, cité à la p. 91, m'est inconnue].

Swinton, A.-H. [L'édition que je possède de son ouvrage intitulé : Insect Variety, cité à la p. 92, ne porte pas de date de publication].

Thylesius, A. [La date de publication de son mémoire intitulé : De Cicindela, cité à la p. 93, m’est inconnue]. 


\section{PROPERTY OF
Z. P. METCALF}

\section{SECOND ADDENDA.}

Au moment de l'impression de la fin de ce travail, je recueille le renseignement bibliographique suivant :

Inering. - Ueber eine merküürdige leuchtende Kaferlarve, in Berliner cntomol. Zeitschr., ann. 1887, t. XXXI, $1^{\text {er }}$ cah.

MoInike, 0. - Phosphorescence d'une espèce nouvelle de Carabide du genre Physodera (Physodera noctiluca, Mohn.), in Sitzungsberichte der niederrhein. Gesellsch. für Natur- und Heilkunde zu Bonn. (Verhandl. des naturhistorisch. Verein. der preussisch. Rheinlande und Westfalens. Bonn), ann. 1875 (séance du 7 juin 1875), p. 154.

[Analyse in BertKau, P.-Bericht über Entomol., Berlin, ann. 1875 et 1876, p. $157(365)]$. 



\section{TABLE DES MATIËRES.}

Avant-propos. . . . . . . . . . . . . . 7

Notes complèmentaires . . . . . . . . . 11

Bibliographie génèrale des Insectes phosphorescents (Anatomie, Physiologie et Biologic) :

Introduction . . . . . . . . . . . . . 27

Bibliographie génèrale . . . . . . . . . . 35

Supplément :

Observations et Rectifications. . . . . . . . . 101

Addenda . . . . . . . . . . . . . 103

Table alphabétique des noms d'auteurs. . . . . . . 109

Table alphabétique des noms d'auteurs par groupe d'Insectes phosphorescents. . . . . . . . . . . . 115

Table alphabétique des noms d'auteurs par date de publication de leurs travaux sur les Insectes phosphorescents.

Observations diverses relatives à la table alphabétique des noms d'auteurs par date de publication de leurs travaux sur les Insectes phosphorescents. . . . . . . . . 132

Second Addenda. . . . . . . . . . . . . . . 133 


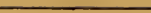

ROUEN. - IMPRIMERIE JULIEN LECERF. 


PROPERTY OF Z.P. METCALF 
